\title{
Smart Grid Data Communication and Analytics
}

\author{
by
}

\begin{abstract}
Arslan Ahmed, B.Sc.
A thesis submitted to the

Faculty of Graduate and Postdoctoral Affairs

in partial fulfillment of the requirements for the degree of
\end{abstract}

Master of Applied Science in Electrical Engineering

Ottawa-Carleton Institute for Electrical and Computer Engineering

Department of Systems and Computer Engineering

Carleton University

Ottawa, Ontario

November 28, 2018

(c) Copyright

Arslan Ahmed, 2018 


\section{Abstract}

With the popularity of smart electrical appliances and home energy management systems, there have been a massive amount of data generated about the electricity consumption. This data can be beneficial for the utility companies as it provides the behaviour patterns of customers, and thus useful decisions can be made to optimize the load on the grid. In this work, we propose and implement a two-way communication system between the transformer agent (TA), attached to a neighbourhood's electric transformer, and its customer agents $(\mathrm{CAs})$, attached to each house in that neighbourhood. Once data is collected at the TA, it is communicated to the utility which can control and suggest any changes in consumption behaviours. In our system, CAs form a self-healing mesh network with the TA using IP-based Wi-Fi, while TAs communicate with the utility headquarters using the LTE network. Our system is implemented in compliance with the IEEE 2030.5 standard requirements, also known as smart energy profile 2.0. We have performed several tests across the Carleton University campus. We have also tested and implemented this system in real neighbourhoods in Ottawa, including Sandcherry and Viewmount sites to prove the system's operation and reliability.

The data obtained from the communication system is stored in a database hosted by IBM Cloud services. Our aim in this work is not only to communicate the data but 
to further process it and help the utility companies design better demand side management (DSM) programs to ensure efficient transmission and distribution of energy. This solves the problem of balancing electric demand and supply at the grid and also reduces peak demands, which helps lower the electricity bills for the consumers. In this context, we analyze the household electricity consumption data to forecast energy consumption for short-term (hours/days ahead) and long-term (weeks/months ahead). To this end, we use and compare seven different machine learning models predicting the energy consumption: linear regression, polynomial regression, support vector regression (SVR) using linear kernel, SVR using Gaussian kernel(SVR-G), SVR using the polynomial kernel, feed-forward neural networks (FFNN), and recurrent neural networks (RNN) using long-short-term memory (LSTM) neurons. To measure the accuracy of these models, we compute three different error metrics: the normalized mean absolute percentage error (NMAPE), the normalized root mean square error (NRMSE), and $\mathrm{R}^{2}$ also known as the coefficient of determination. We then propose a novel approach for short-term load forecasting by combining the power of multiple models and evaluate its performance on a real energy consumption dataset that is publicly available by Massachusetts Institute of Technology (MIT). Results show that our proposed model performs better than existing models for time series energy forecasting. 


\section{Dedicated to my beloved family.}




\section{Acknowledgments}

I would like to express my special gratitude to my supervisor, Prof. Mohamed Ibnkahla, who provided me unparallel academic and moral support throughout the road to finish this thesis. I would also like to extend my warm thanks to Dr. Zied Bouida for his professional mentorship, and always being a source of motivation and encouragement. My special thanks goes to Dr. Waleed Ejaz who supported and guided me through every thick and thin during my stay in Canada. Moreover, I am grateful to my friends at the Sensor Systems and IoT lab for their continuous support.

My sincere appreciation goes to Raed Abdullah from Hydro Ottawa Ltd. for his valuable guidance as a mentor. I cannot thank enough to Dr. Javad Fatthi from University of Ottawa for his generous help and contributions, both as a friend and as

a colleague. It's a great honor to have Prof. Henry Schriemer, Prof. Ashraf Matrawy and Prof. Chung-Horng Lung as my thesis committee members. Their valuable comments and positive criticism added a great value to my thesis. I am also grateful to Prof. Olga Baysal and Prof. Ana-Maria Cretu for providing academic assistance.

My utmost thanks goes to the Pakistani community for their support in difficult times. Most importantly, my praise and thanks is to Allah Almighty, who blessed me with the strength to complete this research. Last but not least, I would like to express my profound gratitude to my loving and caring family for their prayers and support, without which this accomplishment would not have been possible. 


\section{Table of Contents}

$\begin{array}{ll}\text { Abstract } & \text { ii }\end{array}$

Acknowledgments $\quad$ v

Table of Contents vi

List of Tables $\quad$ ix

List of Figures $\quad$ xi

Nomenclature $\quad$ xiii

1 Introduction 1

1.1 Motivation . . . . . . . . . . . . . . . . . . . . 1

1.2 Research Contributions . . . . . . . . . . . . . . . . . . 3

1.3 Thesis Outline . . . . . . . . . . . . . . . . . . . . 3

2 Background 4

2.1 IoT and Smart Grid . . . . . . . . . . . . . . 4

2.1 .1 Introduction to IoT . . . . . . . . . . . . . . . 4

2.1.2 Smart Grid in the Context of IoT . . . . . . . . . . 5

2.2 Smart Grid Communications and Deployment . . . . . . . 6 
2.2.1 The Grid Edge Active Transactional Demand Response (TGDR) Project . . . . . . . . . . . . . 6

2.2.2 IEEE 2030.5 Standard for Smart Grid _ . . . . . . . . 7

2.2.3 Smart Grid Communications in TGDR . . . . . . . . . 8

2.2 .4 Key Performance Indicators . . . . . . . . . . . . . . . . 12

2.2.5 Related Work on Data Communications . . . . . . . . . . 13

2.3 Energy Forecasting . . . . . . . . . . . . . . . . . . . . . 15

2.3 .1 Time Series Data . . . . . . . . . . . . . . . . . . . . 15

2.3 .2 Prediction Models . . . . . . . . . . . . . . . . . . . . . . . . 18

2.3 .3 Evaluation metrics . . . . . . . . . . . . . . . . 27

2.3.4 Related Work on Energy Forecasting . . . . . . . . . . 28

2.4 Summary . . . . . . . . . . . . . . . . . . . 31

3 Implementation of the Smart Grid Communication System 32

3.1 Introduction . . . . . . . . . . . . . . . . . . . 32

3.2 System Model . . . . . . . . . . . . . . . . . . . . . . . . . . . . . 32

3.2.1 Communication between CAs and TA . . . . . . . . . 34

3.2.2 Communication between TAs and Cloud . . . . . . . . . 35

3.2.3 System Compliance with IEEE 2030.5 Standard . . . . . . . 35

3.2.4 Selected Hardware and Implementation Tools . . . . . . . . . 41

3.3 Site Testing Results and Discussions _. . . . . . . . . . . . 43

3.3.1 Carleton University . . . . . . . . . . . . . . . . . . . 44

3.3 .2 Sandcherry Site . . . . . . . . . . . . . . . 56

3.3 .3 Viewmount Site . . . . . . . . . . . . . . . . . . . 65

3.4 Summary . . . . . . . . . . . . . . . . . . . 67 
4 Smart Grid Data Analytics $\quad 68$

4.1 Introduction . . . . . . . . . . . . . . . . 68

4.2 Prediction Engine . . . . . . . . . . . . . . . . . . . 69

4.2 .1 Dataset ............................ 69

4.2.2 Data Visualization . . . . . . . . . . . . . . . . 71

4.2.3 Data Preparation and Pre-processing . . . . . . . . . . . 74

4.2.4 Prediction Scenarios . . . . . . . . . . . . . . . . 81

4.3 Results and Discussions . . . . . . . . . . . . . . . . 82

4.3.1 Scenario-1 (Daily data) . . . . . . . . . . . 82

4.3.2 Scenario-2 (Half-hourly data) . . . . . . . . . . . 87

4.4 Summary . . . . . . . . . . . . . . . . 100

5 Conclusions and Future Work 102

5.1 Conclusions . . . . . . . . . . . . . . . . . . . . 102

5.2 Future Work . . . . . . . . . . . . . . . . . . 104

$\begin{array}{ll}\text { List of Publications } & 105\end{array}$

$\begin{array}{ll}\text { List of References } & 107\end{array}$ 


\section{List of Tables}

2.1 Symbols and descriptions used in prediction models. . . . . . . . . . . 19

3.1 Parameters for Test-5. . . . . . . . . . . . . . . . 51

3.2 Network performance for Test-5: Line of sight. . . . . . . . . . . . 52

3.3 Cases for Test-5: No line of sight. . . . . . . . . . . . . 53

3.4 Network performance for Test-5: No line of sight. . . . . . . . . . 53

3.5 Network performance for Test-6. . . . . . . . . . . . . . . 54

3.6 Distance table for Sandcherry site (meters). . . . . . . . . . . 58

3.7 Parameters for Sandcherry site testing. . . . . . . . . . . . . 59

3.8 Communication from TA to $H_{10}$ without external antenna . . . . . . 60

3.9 Signal strength (in dBm) between the nodes for Test-2. . . . . . . . . 61

3.10 Network performance from TA to various destinations for Test-2. . . . 61

3.11 Results for Test-3 at Sandcherry (refer to Fig 3.15 for TA positions). $\quad 63$

3.12 Signal strength (in dBm) from TA to various destinations for Test-4. 64

3.13 Network performance from TA to various destinations for Test-4. . . . 64

3.14 Signal strength $($ in $\mathrm{dBm})$ for Viewmount site. . . . . . . . . . . 66

3.15 Network Performance from TA to various destinations: Viewmount site. 67

4.1 List of features. . . . . . . . . . . . . . . . . . 77

4.2 One hot encoding of categorical features. . . . . . . . . . . . . . 79

4.3 Evaluation of prediction models for Scenario-1 (Daily data). . . . . . 83 
4.4 Values considered for number of neurons and layers. . . . . . . . . . . 90

4.5 Effect of learning rate. . . . . . . . . . . . . . . . . . . 92

4.6 Evaluation of prediction models for Scenario-2 (Half-hourly data). . . 98 


\section{List of Figures}

2.1 TGDR architecture with our part highlighted. . . . . . . . . . 8

2.2 MPRs tranmission in OLSR protocol. . . . . . . . . . . . . . . . 11

2.3 Weekly decomposition of energy consumption data. . . . . . . . . . 17

2.4 Feed-forward neural network. . . . . . . . . . . . . . . . . 21

2.5 RNN being unrolled into a full network. . . . . . . . . . . . . 23

2.6 Graphical illustration of bias and variance. . . . . . . . . . . . 25

3.1 System model for smart grid communication system. . . . . . . . . . 33

3.2 Multi-hop mesh network between the homes and the local transformer. 37

3.3 Decryption time vs key length. . . . . . . . . . . . . . . . . 40

3.4 Original and encrypted XML files. . . . . . . . . . . . . 45

3.5 HTTPS communication between two nodes. . . . . . . . . . . . . 46

3.6 Initial setup for Test-1. . . . . . . . . . . . . . . . . . . 47

3.7 Placement of nodes for Test-2. . . . . . . . . . . . . . . 48

3.8 Placement of nodes for Test-3. . . . . . . . . . . . . . . . . . 49

3.9 Omni-directional antenna pattern. . . . . . . . . . . . 51

3.10 Placement of nodes for Test-5 and Test-6. . . . . . . . . . . . . . 52

3.11 Node-Red application to connect TA and the cloud. . . . . . . . . . 56

3.12 Front view of Sandcherry site. . . . . . . . . . . . . . . 57

3.13 Back view of Sandcherry site. . . . . . . . . . . . . . 57 
3.14 Top view of Sandcherry site . . . . . . . . . . . . . . 58

3.15 Various TA positions for Test-3 at Sandcherry site. . . . . . . . . 63

3.16 Layout of Viewmount site. . . . . . . . . . . . . . . . 66

4.1 System model for data processing and analytics. . . . . . . . . . . 69

4.2 Energy consumption as a function of different parameters. . . . . . 70

4.3 Real-time power consumption. . . . . . . . . . . . . . . . . 71

4.4 Comparison of current power consumption of four neighbouring houses. 72

4.5 Highest and lowest loads in the neighbourhood, along with warnings from the utility to the houses. . . . . . . . . . . . . . . 72

4.6 Tweet by the utility if outage is predicted in a given neighbourhood. . 73

4.7 Power consumption displayed for different time scales. . . . . . . . . 73

4.8 Histogram of energy data before and removing outliers. . . . . . . 76

4.9 Heat-map of feature correlation. . . . . . . . . . . . . . 78

4.10 Small-scale: predicting next three days based on past two weeks. . . . 84

4.11 Medium-scale: predicting next week based on past month. . . . . . 85

4.12 Large-scale: predicting next month based on past four months . . . . 86

4.13 Short-term forecasting using LSTM. . . . . . . . . . . . . . . 88

4.14 Effect of changing neurons and layers for LSTM. . . . . . . . . . . 89

4.15 Comparison between Adam and SGD optimizer. . . . . . . . . . 92

4.16 Autocorrelation plot. . . . . . . . . . . . . . . . 95

4.17 Accuracy vs history size for different forecasting sizes. . . . . . . . 96

4.18 Short-term forecasting - LSTM vs SVR-G. . . . . . . . . . . 97

4.19 Stacking block diagram. . . . . . . . . . . . . . . 97

4.20 Performance of our proposed ensemble model vs existing models. . . . 99

4.21 Error vs forecasting size using different error metrics. . . . . . . . . 100 


\title{
Nomenclature
}

\author{
Notation Description
}

Adam Adaptive Moment Estimation

AP Access Points

API Application Program Interface

BOS Back Office System

BPTT Back-propagation Through Time

CA Customer Agent

COD Coefficient Of Determination

DL Downlink

DR Demand Response

DSM Demand Side Management

DSO Distributed System Operator

EMS Energy Management System 


\begin{tabular}{|c|c|}
\hline EXI & Efficient Extensible Interchange \\
\hline FFNN & Feed-Forward Neural Networks \\
\hline HEMSC & Home Energy Management System Controller \\
\hline HEMS & Home Energy Management System \\
\hline HOL & Hydro Ottawa Limited \\
\hline HSMM & High-Speed Multimedia Mesh \\
\hline HTTP & HyperText Transfer Protocol \\
\hline HTTPS & Hyper Text Transfer Protocol Secure \\
\hline IEEE & Institute of Electrical and Electronics Engineers \\
\hline IoT & Internet of Things \\
\hline IP & Internet Protocol \\
\hline KPI & Key Performance Indicator \\
\hline LP & Linear Programming \\
\hline LSTM & Long-Short-Term Memory \\
\hline LTE & Long Term Evolution \\
\hline MANET & Mobile Ad-Hoc Network \\
\hline MPR & Multi-Point Relay \\
\hline
\end{tabular}




\begin{tabular}{|c|c|}
\hline NEMA & National Electrical Manufacturers Association \\
\hline NMAE & Normalized Mean Absolute Error \\
\hline NRMSE & Normalized Root Mean Square Error \\
\hline OESC & Ontario Electrical Safety Code \\
\hline OLSR & Optimized Link State Routing \\
\hline OSPF & Open Shortest Path First \\
\hline REST & Representational State Transfer \\
\hline $\mathrm{RF}$ & Radio Frequency \\
\hline RFE & Recursive Feature Elimination \\
\hline RNN & Recurrent Neural Networks \\
\hline RSA & Ron Rivest, Adi Shamir and Leonard Adleman \\
\hline SCP & Secure Copy \\
\hline SEP & Smart Energy Profile \\
\hline SGD & Stochastic Gradient Descent \\
\hline $\mathrm{SSH}$ & Secure Shell protocol \\
\hline SVR & Support Vector Regression \\
\hline SVR-G & SVR using Gaussian kernel \\
\hline & Transformer Agent \\
\hline
\end{tabular}


TGDR The Grid Edge Active Transactional Demand Response

TLS Transport Layer Security

UMASS University of Massachusetts at Amherst

UL Uplink

VANET Vehicular Ad-Hoc Network

WMN Wireless Mesh Networks

XML Extensible Markup Language 


\section{Chapter 1}

\section{Introduction}

According to the 2015 report by the United Nations, the world's population will reach 9.7 billion people by 2050 [1]. As people move to more populated areas, cities are facing the challenges of providing enough power to accommodate everyone while using outdated power grids. The Internet of Things (IoT) and big data revolution has the power to reshape the way we think about cities across the world. It is connecting people and governments to smart city solutions with the help of smart grid technology, designed to improve upon and replace the older architecture. These technologies promise a smart grid that is more resilient and cost-effective than the current power infrastructure. However, it comes with many challenges as well as opportunities, which are discussed next in this chapter. We then present a summary of our key contributions towards solving these challenges, followed by the outline of this thesis.

\subsection{Motivation}

The concept of smart grid systems has been around for a while, and the recent technological advancement in communications and sensing areas have further the 
development of these systems. The traditional power grid consists of centralized generation, where energy is pushed one-way through transmission and distribution networks to the end users. Smart grids add bi-directional communication between the utility and the consumers with the options of renewable energy generation and storage. On top of the existing power network layer, there is a new communication layer for information exchange and control [2]. Balancing of electric supply and demand at the grid has always been a big challenge for electric utility companies. To counter this, utilities design demand response (DR) and demand-side management (DSM) programs. This encourages consumers to modify their electricity consumption habits through various methods including financial incentives and behavioural change. With the increasing penetration of advanced sensors in power systems, an influx of huge datasets presents a valuable opportunity to gain insights for improving system operation and planning in the context of the electric grids. The four Vs of big data, i.e. volume, velocity, variety, and veracity [3] empower the utilities to make better decisions in order to optimize the load at the grid. Modern statistical techniques for data exploration enable them to better understand customer's usage pattern, forecast energy consumption, and make recommendations to customers based on their real-time behaviour. It also helps utilities predict the downtime and power failures, act proactively, and negotiate the pricing with the end users as explained in [4]. Furthermore, it provides the opportunity to consumers to adjust their loads according to dynamic pricing in order to minimize their monthly bills [5]. All this motivates us to work on smart grid communications and data analytics, which is the main area of focus for this thesis. 


\subsection{Research Contributions}

In light of the above, the main contributions of this thesis are summarized as follows:

- Implemented a secure and reliable bi-directional communication system between the customer agents (CA) at the homes, the transformer agent (TA) serving a given neighbourhood, and the back office system (BOS). This real-field demonstration of smart grid technology is first implementation compliant with the IEEE 2030.5 standard. This standard outlines the constraints in terms of security, message formatting, and many other aspects to maintain interoperability between various smart grid systems implemented across the globe.

- Analyzed time series data for electricity consumption and compared various data prediction models. Proposed a novel approach for short-term electricity forecasting based on ensemble modelling, that outperforms the baseline models.

\subsection{Thesis Outline}

This thesis is organized as follows: Chapter 2 provides background on IoT and smart grids and the context of the proposed work. Chapter 3 gives the details behind our testing and implementation of smart grid communication system in real neighbourhoods. In Chapter 4, we apply and compare various machine learning models for short-term and long-term forecasting for energy consumption data. We further present our proposed approach based on ensemble model of LSTM and SVR-G. Finally, Chapter 5 concludes this thesis along with recommendations for future work. 


\section{Chapter 2}

\section{Background}

In this chapter, we provide background about the IoT, smart grids, and the project that our work is done under. We also discuss big data sources and prediction models used in the smart grid. The content presented in this chapter aims at providing relevant context to the discussion in the following chapters.

\subsection{IoT and Smart Grid}

\subsubsection{Introduction to IoT}

IoT is the interconnection of intelligent devices and management platforms that, with little to no human intervention, collectively facilitates a smart connected world [6]. From health monitoring to smart utilities, and from integrated logistics to autonomous drones, our world is becoming hyper-automated. The unprecedented growth in IoT communications is predicted to accumulate to over 20 billion connected IoT devices by 2020 , which are anticipated to generate annual revenue of USD 8.9 trillion $[7,8]$. Applications for the IoT not only emphasize the nonexistence of human intervention but also the autonomous nature of machines. A new wave of the industrial revolution is driven by the dramatic altering of manufacturing, energy, transportation, 
medical, and other industrial and municipal sectors. This revolution is facilitated by the collection, aggregation, and analysis of sensor and device data to maximize the efficiency of machines and the throughput of operations and processes. IoT applications span motion control, machine-to-machine interactions, predictive maintenance, smart-grid energy management, big data analytics, smart cities, and interconnected medical systems $[9,10]$.

\subsubsection{Smart Grid in the Context of IoT}

Smart grid is one of the key applications of IoT which falls under the umbrella of smart cities. According to the International Energy Agency, the global energy demand is set to grow by $37 \%$ from 2014 to 2040 where electricity is the fastest growing final form of energy [11]. Thus, meeting increased demand for electricity is considered as one the most critical challenges facing modern societies. Efforts initiated to meet this challenge include generating electricity from renewable energy sources, such as solar and wind; developing energy storage systems; modernizing and optimizing the electric grids; and increasing consumer awareness and participation in achieving energy sustainability. The modern electric grid, also referred to as the "smart grid", is equipped with advanced instrumentation for monitoring, control, and communication. Among the significant characteristics of the smart grid are: widespread deployment of smart meters to collect fine grained electricity consumption information, integration of renewable energy sources, rise in distributed energy generation, increased use of electric vehicles, customer engagement using demand response and time of use pricing, microgrids, and electricity markets [12]. The U.S. Department

of Energy formally describes the smart grid as: A fully automated power delivery network that monitors and controls every customer and node, ensuring a two-way flow of electricity and information between the power plant and the appliance, and 
all points in between. Its distributed intelligence, coupled with broadband communications and automated control systems, enables real-time market transactions and seamless interfaces among people, buildings, industrial plants, generation facilities, and the electric network [13].

\subsection{Smart Grid Communications and Deployment}

This work is done in collaboration with Hydro Ottawa Limited (HOL) under "The Grid Edge Active Transactional Demand Response" project known as the The GREAT-DR and denoted by TGDR in this thesis.

\subsubsection{The Grid Edge Active Transactional Demand Response (TGDR) Project}

TGDR is a field demonstration project of smart grid technology based on the IEEE 2030.5 interoperability standard [14]. This project provides interoperable and transactive demand response solution which allows a customer's electricity meter to interact virtually and seamlessly with the electrical grid. This initiative gives participants the tools to manage their electricity usage and demand while taking advantage of home generation and storage solutions [15].

\subsubsection{Objectives of TGDR}

TGDR manages demand response in real-time within the grid's dynamic operating limits through automatic and active negotiation between devices that use or produce electricity at the customer-level as detailed in [4]. This is accomplished at three levels:

- Premise-level: using a home energy management system(HEMS) and CA 
- Local (i.e., neighbourhood) and zonal level: using transactive agents and TA

- Grid level: using a BOS at the utility

\subsubsection{Administration of TGDR}

TGDR project was conceived and initiated by Hydro Ottawa [16], partially funded by the Ontario Ministry of Energy's Smart Grid Fund [17] and the LDC Tomorrow Fund [18]. This project is supported by various collaborators including: Carleton University, University of Ottawa, CIMA Canada, Panasonic Eco Solutions with Tabuchi Americas, Energate, Thorium Technologies, and Quadra Power Systems.

\subsubsection{Thesis as Part of TGDR}

The work done in this thesis has contributed towards a major part for the implementation of the TGDR. These contributions include the design, implementation, and the maintenance of high quality communications based on IEEE 2030.5 standard (details in Section 2.2.2). Figure 2.1 shows the TGDR architecture and highlights our contribution as a part of this project.

\subsubsection{IEEE 2030.5 Standard for Smart Grid}

With the heterogeneity of devices used in smart grids, the era of IoT, the variety of data formats, and the critical impact of security challenges on smart grids, IEEE has lately published IEEE 2030.5 as a standard for smart grids [14]. IEEE 2030.5 is a communication standard for both consumers and the smart grid. The standard complies with the IoT concept and grants consumers the ability to manage energy generation and consumption. Moreover, it helps in the integration of smart devices and 
Summary:

1. This schematic represents some of the components and how they interact within this smart Grid system. A commercially available residence EMS/CDM

controller interacts with the residence panelboard and devices. It manages and operates the residence loads, generation and storage based on the Consumer preferences.

3. The residence EMS/CDM controller communicates filtered data to the Customer Agent. This data is limited to maintain Customer privacy. The long term goal is to integrate the Customer Agent into the EMS/CDM controller.

4. The Customer Agent communicates with the Transformer Agent. The information is limited to what can be offered in kW for how long and when.

5. The Transformer Agent communicates with the various Customer Agents being fed by the same Utility Transformer.

6. The Transformer Agent compiles all the data and sends it to the Utility Substation. All negotiation requests are logged and results for gauging benefits achieved, and future analysis for improvement.

7. The Utility Substation forwards aggregate data to the Utility Head Quarters. When the Grid has loading is problematic, the Utility Head Quarters can send demand response information back to the Transformer Agents.

8. As required, the Transformer Agent dynamically negotiates with the various Customer Agents to meet the Grid or transformer dynamic capacity limit.

Legend:
-1) WiMAX/ wireless from
$\Leftrightarrow \quad$ Internal Power Line Carrie
$\Leftrightarrow \quad$ External Power Line Carrier (filtered)
- Other communication means
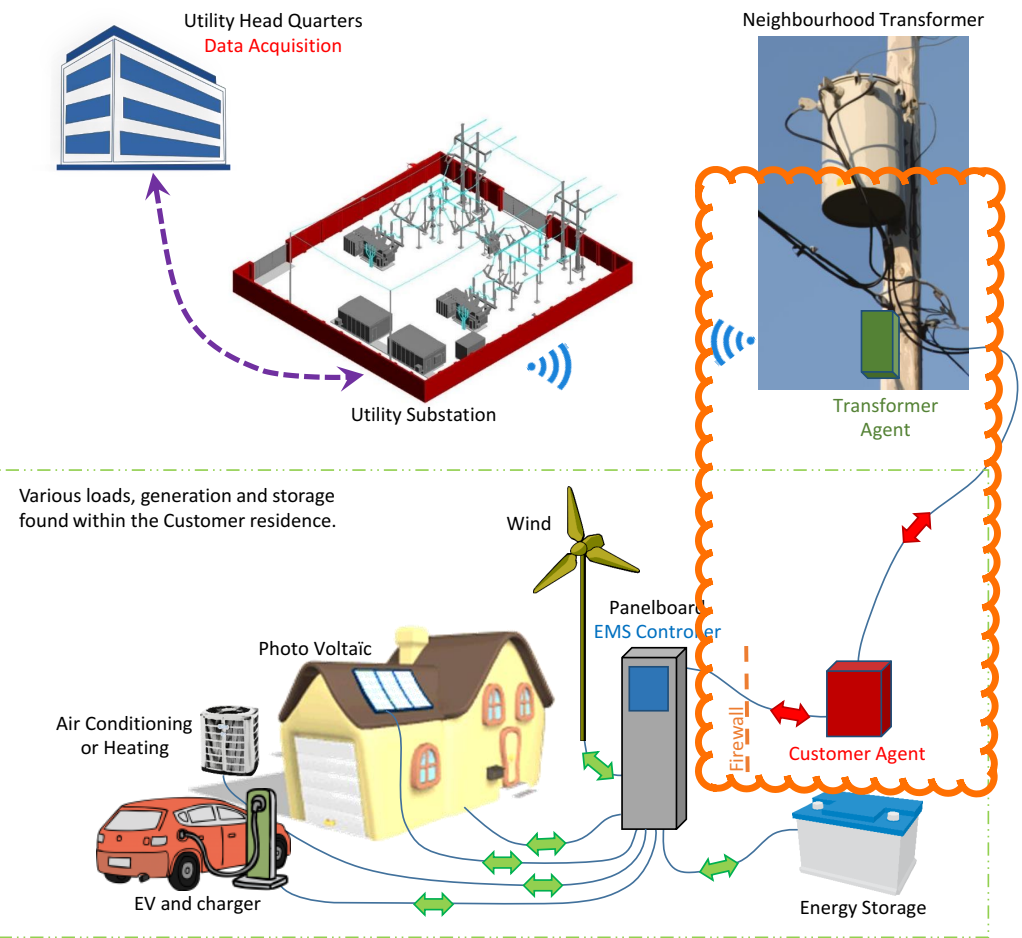

smart applications by defining a framework of secure and interpretable communication. Some of the major requirements of the standard that we take into consideration in our implementation are

- Internet Protocol (IP) based communication

- RESTful HTTP protocol for communications

- TLS 1.2 (HTTPS) based security

- XML and/or EXI format for messages

\subsubsection{Smart Grid Communications in TGDR}

A core element to TGDR's success is the communication system between CAs, TA, and the BOS. This system is based on wireless connections between different agents. 
There are different topologies and routing protocols that govern these wireless networks. In what follows, we provide the details behind these protocols.

\subsubsection{Topology}

Wireless networks typically work in one of two configurations or topologies: ad-hoc or infrastructure. An infrastructure mode network requires the use of an Access Point which controls the Wireless communication between nodes. On the other hand, an ad-hoc network allows the nodes to communicate directly with each other without any central access point controlling the communication. They are simple to set up, inexpensive and fast [19].

The decentralized nature of wireless ad-hoc networks makes them suitable for a variety of applications where central nodes cannot be relied on and may improve the scalability of networks compared to wireless managed networks, though theoretical and practical limits to the overall capacity of such networks have been identified. The presence of dynamic and adaptive routing protocols enables ad-hoc networks to be formed quickly, and they are more reliable as there is no single point of failure. Wireless ad-hoc networks can be further classified by their applications including mobile ad-hoc networks (MANETs), vehicular ad-hoc networks (VANETs), smartphone ad-hoc networks (SPANs), and Ad-hoc home smart lighting [20].

Wireless mesh networks: They come under the form of wireless ad-hoc networks, are more robust than star-based networks, and offer self-healing features [21,22]. Such networks often consist of mesh clients and gateways. Mobility of nodes is less frequent. If nodes constantly or frequently move, the mesh spends more time updating routes than delivering data. In a wireless mesh network, topology tends to be more static, so that routes computation can converge and delivery of data to their destinations can 
occur. Hence, this is a low-mobility centralized form of wireless ad-hoc network. Mesh clients in our case are CAs, and the mesh gateway is the TA. A mesh network is reliable and offers redundancy. When one node can no longer operate, the rest of the nodes can still communicate with each other directly or through one or more intermediate nodes. Wireless mesh networks can self-form and self-heal. They work with different wireless technologies including 802.11, 802.15, 802.16, and cellular technologies and they are not restricted to any one technology or protocol.

\subsubsection{Routing protocol}

Routing is the process of selecting paths in a network to send network traffic. The paths can be calculated using two techniques; distance vector, or link state routing. In the distance vector scheme, each router periodically shares its knowledge about the entire network with its neighbours. On the other hand, in the link state scheme, each router only has information about their neighbours and which nodes are connected to other nodes. In this case, each node independently calculates the next best logical path to every possible destination in the network. Each collection of best paths will then form each node's routing table. However, whenever a change in the connectivity, it is necessary to re-compute the shortest path tree and then recreate the routing table.

Routing is a critical element in mesh topologies and effects its performance [23]. There exist several routing protocols that have been employed in the context of smart grid systems [23, 24]. The OLSR protocol is a proactive link-state routing protocol for mobile and ad-hoc networks [25]. OLSR achieves fast self-healing and reduces the network control overhead compared to other classical link-state routing protocols, like the open shortest path first (OSPF), by selecting multi-point relays (MPRs). The MPRs are selected such that they cover all the nodes that are two-hop neighbours. 
Only MPRs broadcast messages about the link states which reduces the control overhead and makes it more suitable for networks with high mobility or repeated changes in the nodes states.

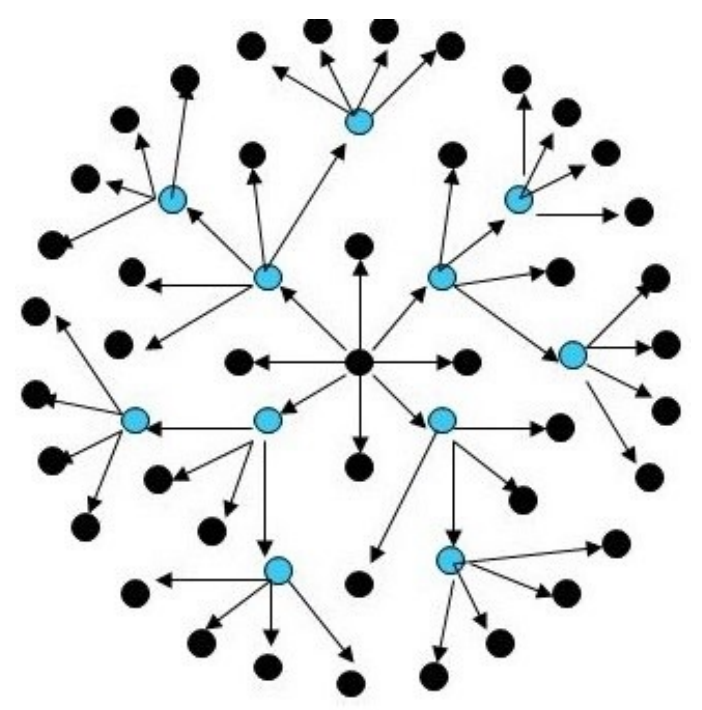

11 retransmissions to send a message up to 3 hops

Figure 2.2: MPRs tranmission in OLSR protocol [26].

OLSR: OLSR is a proactive routing protocol for mobile ad-hoc networks. The protocol inherits the stability of a link state algorithm and has the advantage of having routes immediately available when needed due to its proactive nature. This protocol works in a distributed manner without depending on any central entity. It operates as a table-driven proactive protocol, i.e., regularly exchanges topology information with other nodes of the network. Each node selects a set of its neighbour nodes as MPRs. In OLSR, only selected MPRs nodes are responsible for forwarding control traffic intended for diffusion into the entire network as shown in Fig. 2.2. MPRs provide an efficient mechanism for flooding control traffic by reducing the number of transmissions required [27]. 


\subsubsection{Key Performance Indicators}

There are various key performance indicators (KPIs) to evaluate the performance of a wireless solution. Some of the KPIs are explained below [28]. We will use most of them in our communication tests to establish the authenticity of our system.

Coverage: It is the range of a wireless link to reliably connect devices measured in meters from the gateway to the internal nodes, and varies depending on the radio frequency $(\mathrm{RF})$ propagation environment. Factors, such as terrain, clutter type (urban, suburban, rural), interference, and local output power regulations determine the range capabilities.

Capacity: It is the amount of data from device endpoints a gateway can simultaneously serve. The capacity is measured as the aggregate application throughput (kbps) at the gateway. Coverage and capacity must work in parallel to achieve a highly functioning network. They work in conjunction to drive the ratio of access points (APs) to device endpoints.

Coexistence: It is the ability of a wireless technology to coexist with other devices that can cause significant and dynamic interference. This is particularly important when operating in unlicensed bands.

Power consumption: It is directly related to the life of the battery, and hence the operational costs. The wireless protocol mainly drives the power efficiency. The key to efficiency is to minimize the time and power spent transmitting and receiving. This can be done using various techniques including fast acquisition to minimize the amount of time needed to transmit the packet. Also, the protocol should be designed such that the endpoint is in a low power deep sleep mode most of the time. 
Security: It is the ability of a wireless system to resist malicious attacks from cyber criminals. To prevent malicious attacks, the device networks require proven and robust cyber-security across the network. A strong network should use a comprehensive defence in depth strategy to deliver this information security including:

- Prevention mechanisms: Provide access control, mutual authentication, confidentiality, and high availability.

- Detection mechanisms: Identify system breach attempts and alert operators.

- Recovery mechanisms: Ensure the system degrades gracefully and continued operation even while under attack.

Latency: It is also known as the time delay of communications which includes access and propagation delay.

Access delay: This delay is calculated from the time when data is ready to transmit until it begins transmission. It includes any time required to wait until transmission may begin, logic delays like encoding of the data, and adding error correction.

Propagation latency: It is the travel time through the medium from the sender to the receiver, which is calculated from the first byte transmitted through the medium to the first byte received at the destination.

\subsubsection{Related Work on Data Communications}

In the literature, several contributions considered the implementation of a smart grid communication system using Wi-Fi and ZigBee [29-31]. Zigbee is an IEEE 802.15.4based, low power, low-cost, and two-way wireless communication standard. It normally has transmission range up to $75 \mathrm{~m}$, and supports up to 65000 networked devices, 
and transmission bandwidths of 20-250 kbps depending on the applied industrial, scientific, and medical (ISM) frequency band, i.e. $864,915 \mathrm{MHz}$, and $2.4 \mathrm{GHz}$. On the other hand, WiFi is an IEEE 802.11 wireless local area network (WLAN) standard, also on the ISM frequency band (e.g., 2.4GHz). It provides coverage range from 30 to $100 \mathrm{~m}$, up to 255 connections to an access point (AP), and physical transmission throughput from 11 to $600 \mathrm{mbps}$ depending on the applied standard/technology. At the moment, the main advantage of Zipbee over WiFi is the power consumption [32]. However, this advantage is diminishing as power consumption of WiFi chipsets has been undergoing significant improvements. Moreover, it is non-IP Based and offers limited data rates which make it non-scalable and incompatible with future IoT Networks [33].

Several communication topologies and routing protocols have been employed in the context of smart grid systems [34]. In [35], a hybrid Wi-Fi-LTE aggregation data communication architecture is implemented. In their proposal, a Wi-Fi Mesh network is established between several homes to aggregate energy data and pass it to a central node which will then use LTE to send it to the utility's headquarters. The hybrid network architecture was evaluated using ns-3. Their results showed that the combination of Wi-Fi and LTE can improve control channel and random access channel performance at the cost of tolerable latency degradation. Researchers in [36] further propose the OLSR protocol to route traffic between the nodes in a smart grid. Through examining the performance of the hybrid wireless mesh protocol and OLSR, they concluded that the performance of OLSR protocol is the same or, in some cases, marginally outperforms the IEEE 802.11s standard default protocol. 


\subsection{Energy Forecasting}

IoT and data analytics are core technologies in smart grid that help the service providers store and process data in real time in the cloud. It also helps users to optimize their energy consumption by negotiating with the utility company. Moreover, statistical techniques for data exploration and energy forecasting enable the utility to better understand customers' behaviour, keep track and predict the downtime and power failures, act proactively, and negotiate with the end users in spatial and temporal dimensions [37]. Here in this work, we will explore and forecast the energy consumption data as explained in Chapter 4.

\subsubsection{Time Series Data}

\subsubsection{Introduction and decomposition}

Energy consumption data is time series data by nature, which means it is a set of data points collected at constant time intervals. The application of time series forecasting and analysis spans across multiple domains and businesses. It is used in statistics, signal processing, pattern recognition, econometrics, mathematical finance, weather forecasting, earthquake prediction, electroencephalography, control engineering, astronomy, and communications engineering.

The uniqueness about time series data is that it is time-dependent, so the underlying assumption of a linear regression model that the observations are independent does not hold in this case. Time series analysis accounts for the fact that data points taken over time may have an internal structure (such as autocorrelations, trend, and seasonality) that should be accounted for. This is explained in detail below in the form of various components of time series:

- Trend: The general increase or decrease in the series over a period of time, 
for example the number of air passengers has overall increased for the past five years.

- Seasonality: The up and down fluctuation that re-occurs each period (month, year etc.), for example, people tend to consume more energy during certain hours of the day, and this pattern repeats daily.

- Residual effect: What remains after removing the trend and the seasonal components of a time series. It represents the random error effect of a time series, caused by the randomness of human actions or any unexplained causal factors. This effect is for short duration and non-repeating.

Splitting a time series into these three components is called decomposition. Decomposed time series data for our energy dataset is shown in Fig 2.3. Here we see the decomposition based on weekly interval. Observing the seasonal data, we can conclude that there is generally more energy consumed on weekends and Mondays as compared to other days. Similarly, we can draw other conclusions based on analytics.

\subsubsection{Strategies for forecasting}

In time series forecasting, we always forecast the next unknown data point in a time series data; this is known as one-step forecasting. For example, given half-hourly data for a certain number of previous days, we forecast the next half hour data (i.e. one next step). There has been a lot of study to solve challenges faced in multi-step ahead forecasting. Unlike one-step ahead forecasting, multi-step ahead forecasting tasks are more difficult since they have to deal with various additional complications like the accumulation of errors, reduced accuracy, and increased uncertainty [38]. 

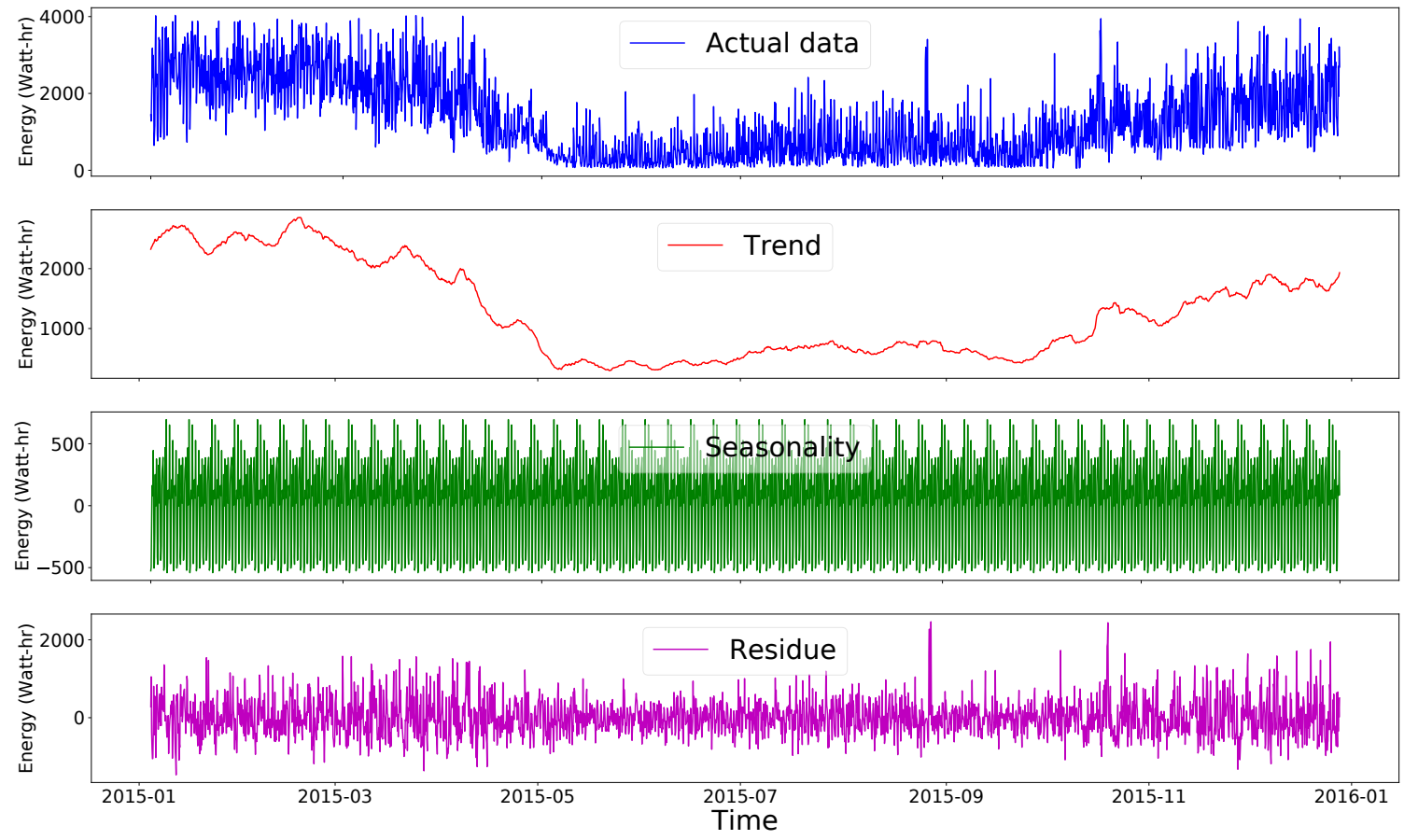

Figure 2.3: Weekly decomposition of energy consumption data.

There are many different strategies for multi-step time series forecasting which can be divided into two main categories [39].

- Direct multi-step forecast: Here we forecast each step independently using a separate model, therefore to forecast for $x$ steps, we need $x$ separate models

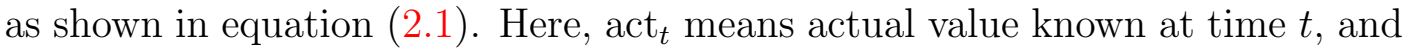
$n$ is the size of history window we are using to forecast the next point.

$$
\begin{aligned}
& \text { Forecast }_{t+1}=\operatorname{model}_{1}\left(\operatorname{act}_{t}, \operatorname{act}_{t-1}, \ldots, \operatorname{act}_{t-n}\right), \\
& \text { Forecast }_{t+2}=\operatorname{model}_{2}\left(\operatorname{act}_{t-1}, \operatorname{act}_{t-2}, \ldots, \operatorname{act}_{t-n}\right), \\
& \text { Forecast }_{t+3}=\operatorname{model}_{3}\left(\operatorname{act}_{t-2}, \operatorname{act}_{t-3}, \ldots, \operatorname{act}_{t-n}\right) .
\end{aligned}
$$

- Recursive multi-step forecast: Here, we forecast one next step and use this forecasted value to forecast another step. This way, we can use a single trained 
model to forecast multi-step as shown in equation (2.2). The size of the history window remains constant untill we have predicted the last step. In this case, if we are using five past values to forecast the next value at time $t+1$, we will still be using the total of five past values to forecast the value at time $t+2$, but four of them are actual past values and one is the predicted value at time $t+1$. Similarly, to forecast at time $t+3$, we now use past two predicted values $(t+2$ and $t+1)$, and past three actual values to keep the history window size constant at five. This defines the sliding window concept. This model is the most commonly used strategy to forecast time series data in the literature, and we will use the same in our work.

$$
\begin{aligned}
& \text { Forecast }_{t+1}=\operatorname{model}\left(\operatorname{act}_{t}, \operatorname{act}_{t-1}, \ldots, \operatorname{act}_{t-n}\right) \\
& \text { Forecast }_{t+2}=\operatorname{model}\left(\text { Forecast }_{t+1}, \operatorname{act}_{t}, \operatorname{act}_{t-1}, \ldots, \operatorname{act}_{t-n}\right) \\
& \text { Forecast }_{t+3}=\operatorname{model}\left(\text { Forecast }_{t+2}, \text { Forecast }_{t+1}, \operatorname{act}_{t}, \ldots, \text { act }_{t-n}\right) .
\end{aligned}
$$

The disadvantage of using direct multi-step forecast is that it is computationally expensive as we have to train a separate model for every forecasting step. Moreover, separate models also do not consider the complex dependencies between the forecasted values. However, since there is an independent forecast for each of the steps, we can avoid the error accumulation problem faced in Recursive strategy. However, we can neglect the accumulation of errors since we are forecasting only for short-term.

\subsubsection{Prediction Models}

We use several prediction techniques that we explain below with the used notations defined in Table 2.1. 
Table 2.1: Symbols and descriptions used in prediction models.

\begin{tabular}{||c|c||}
\hline Symbol & Description \\
\hline \hline$x$ & Training/Testing input data \\
\hline$y$ & Training/Testing output data \\
\hline$\hat{y}$ & Prediction error \\
\hline$Y$ & Regression model \\
\hline$x_{k}$ & Variable dependent on function's order \\
\hline$J$ & Cost function \\
\hline$Z(x, y)$ & Function output \\
\hline$\sigma$ & Adjustable parameter \\
\hline$n$ & Polynomial order \\
\hline$\alpha, c, m, b_{k}$ & Constants \\
\hline
\end{tabular}

\subsubsection{Linear and Polynomial Regressions}

Linear Regression: The linear relationship between variables is mapped with a line equation with a certain slope using the linear relationship $Y=b_{0}+b_{1} x_{1}$. Linear regression is typically used to predict uniform and linear data that does not allow for more complex forecasts.

Polynomial Regression: In regression analysis, polynomial regression intakes a predictive path of the $n$th order as given in the following representation

$$
Y=b_{0}+b_{1} x_{1}+b_{2} x_{2}^{2}+\cdots+b_{n} x_{n}^{n} .
$$

Similar to linear regression, polynomial regression maps energy readings with slightly additional complexity. 


\subsubsection{Support Vector Regression (SVR)}

Linear kernels: The linear kernel, defined in equation (2.4), is one of the simplest types of SVR kernels for prediction. It is defined as the dot product of two vectors in a higher dimensional feature space. Linear kernels are extremely useful when dealing with datasets that are linearly separable.

$$
Z(x, y)=x^{T} y+c .
$$

Polynomial kernels: Regression problems that are not linearly separable require the use of non-linear kernels. Polynomial Kernels used in SVRs are useful in this case by projecting the features into several higher dimensions, then imposing polynomial regression, and creating a hyperplane to separate the data. These kernels can be given as

$$
Z(x, y)=\left(a x^{T} y+c\right)^{d} .
$$

RBF or gaussian kernels: The RBF kernel, as defined in equation (2.6), takes the form of a radial basis or a Gaussian function.

$$
Z(x, y)=\exp \left(-\frac{\|x-y\|^{2}}{2 \sigma^{2}}\right) .
$$

While RBF kernels are widely implemented due to their easily calibrated parameters and reasonable point of reference in terms of comparison between energy consumption predictions in other SVR kernels.

Different kernels can be used for different scenarios and this is a significant step in the process of deciding on which algorithm to approach a specific dataset with. Each dataset is unique in terms of its features. If data is linearly separable then a linear kernel would be suitable, yet if it is not, the other functions such as the polynomial or 


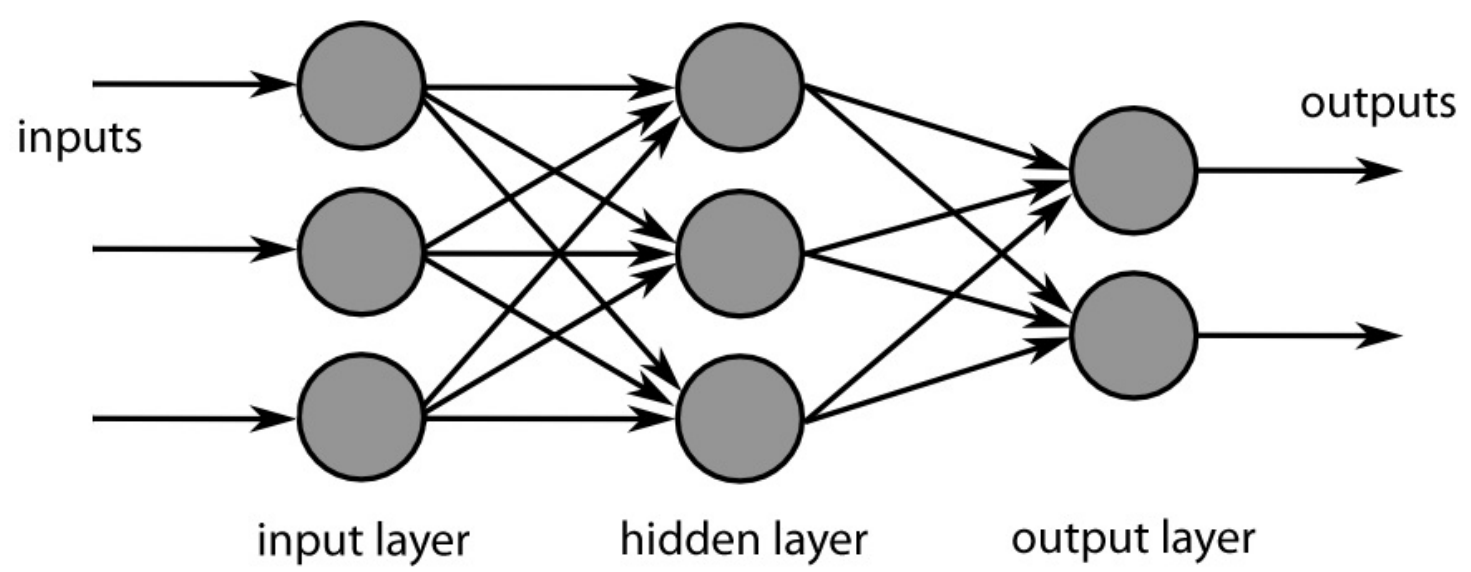

Figure 2.4: Feed-forward neural network.

RBF kernels can be introduced. Keeping in mind that support vector regression does not only operate in its base-dimensions, yet it can use higher dimensions to exploit the feature's positioning in a particular space and this is very useful when working with highly complex data.

\subsubsection{Feed-forward neural network}

Neural Networks are widely used for many classification and regression problems in machine learning. Feed-Forward Neural Networks (FFNNs) have been applied to time-series prediction for datasets that contain interval-based data. Applications of FFNNs with time-series range from stock prices to smart city energy consumption [40]. A basic feed-forward neural network is shown in Fig 2.4.

FFNN model is based on two input nodes; consuming normalized time and temperature. FFNN uses a sigmoid activation function $f(x)=1 /\left(1+e^{-x}\right)$ which is a real-valued differentiable function used also in artificial neural network to quantify non-linearity in a model. The cost function, $J=\sum 1 / 2(y-\hat{y})^{2}$, is then minimized 
using stochastic gradient descent which avoids unwanted higher-dimensional computational complexity.

\subsubsection{Recurrent neural network with LSTM neurons}

Recurrent neural networks with LSTM Cells are a specific type of artificial neural networks. RNNs are different from FFNN in terms of complexity and this due to the RNNs recurrent nature. In FFNN, data is generally only propagated forward, whereas in RNNs, data is processed recursively. The RNNs are designed in way that associates them with a "recurrent" element, whereas each block uses the output from the previous RNN block as an input. This allows for the induction of a memory gradient (i.e. retaining information through time) as shown in Fig 2.5 [41].

\section{Back-propagation through time (BPTT) and vanishing gradient problem:}

Error gradients with respect to weights are calculated using BBTT by unfolding the RNN in time and differentiating.

$$
\theta(x)=\frac{1}{1+e^{-x}} .
$$

Here $\theta$ is the activation function, $x_{t}$ is the input, $s_{t}$ is the local output (hidden layer) and $o_{t}$ is the output.

$$
s_{t+1}=\theta\left(W s_{t}+U x_{t+1}+b_{s}\right) .
$$

LSTM cell: A solution to the vanishing gradient problem is through using LSTM units that use different cells as explained below:

- Forget gate layer: The forget gate layer mainly looks at the $h_{t-1}$ and $x_{t}$ 


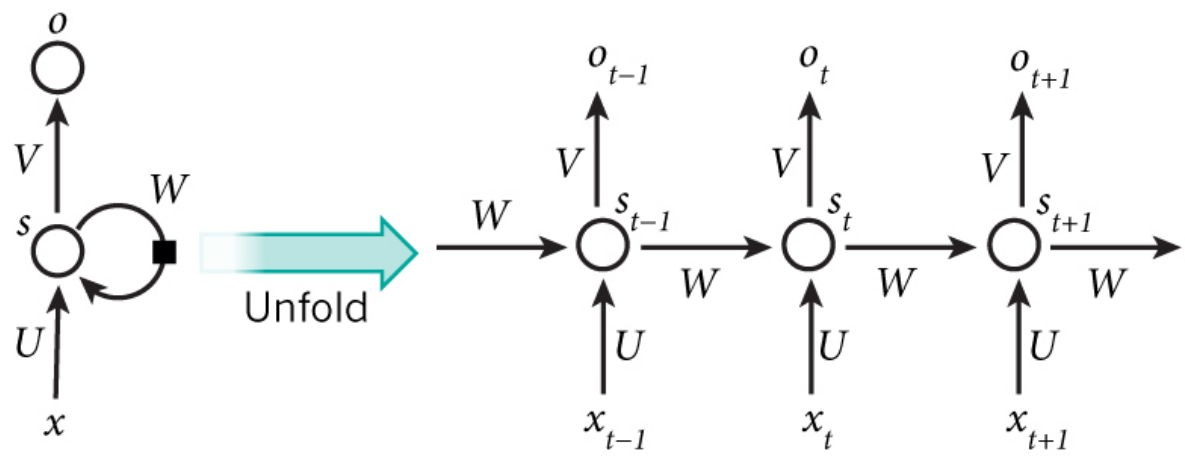

Figure 2.5: RNN being unrolled into a full network [41].

variables and returns a number between 0 and 1 using the sigmoid layer in order to decide on what information should be discarded from the cell.

$$
f_{t}=\sigma\left(W_{f} \cdot\left[h_{t-1}, x_{t}\right]+b_{f}\right)
$$

- Input gate layer: The next step is the input gate layer which decides on which information stays in the cells state. Computations are made at two different distinct steps; a) which values will be updated and b) creation of new candidate values through the tanh layer.

$$
\begin{gathered}
i_{t}=\sigma\left(W_{i} \cdot\left[h_{t-1}, x_{t}\right]+b_{i}\right) . \\
C_{t}=f_{t} * C_{t-1}+i_{t} * C_{t} .
\end{gathered}
$$

- Output layer: Finally, we output the updated version of the previous cell state as the final output based on the following equation.

$$
o_{t}=\sigma\left(W_{o} \cdot\left[h_{t-1}, x_{t}\right]+b_{o}\right) \text {. }
$$




$$
h_{t}=o_{t} * \tanh \left(C_{t}\right) .
$$

\subsubsection{Ensemble modelling}

In machine learning language, ensemble learning means strategically combining multiple models to achieve better prediction accuracy than any of the base models. These models can be different from each other for a variety of reasons, for example, entire hypothesis may be different for two models, or sometimes different variations of a model that mathematically looks the same are actually very different due to the difference in the selected tuning parameters. This gives rise to different errors for different models. The more the diversity in performance of the base models, the better the ensemble model will perform [42].

The error emerging from any model can be broken down into two main components mathematically. Bias error is useful to quantify how much on an average are the predicted values different from the actual value. A high bias error means we have an under-performing model which keeps on missing essential trends. Variance on the other side quantifies how are the prediction made on same observation different from each other. A high variance model will over-fit on your training population and perform poorly on any observation beyond training. Fig 2.6 gives more clarity, assuming that red spot is the real value and blue cross are predictions.

As we increase the complexity of your model, we will see a reduction in error due to lower bias in the model. However, this only happens till a particular point. As we continue to make our model more complex, we end up over-fitting your model and hence the model will start suffering from a high variance. A perfect model should maintain a balance between these two types of errors. Ensemble learning is one way to execute this balance by combining the advantage of various models [43]. 

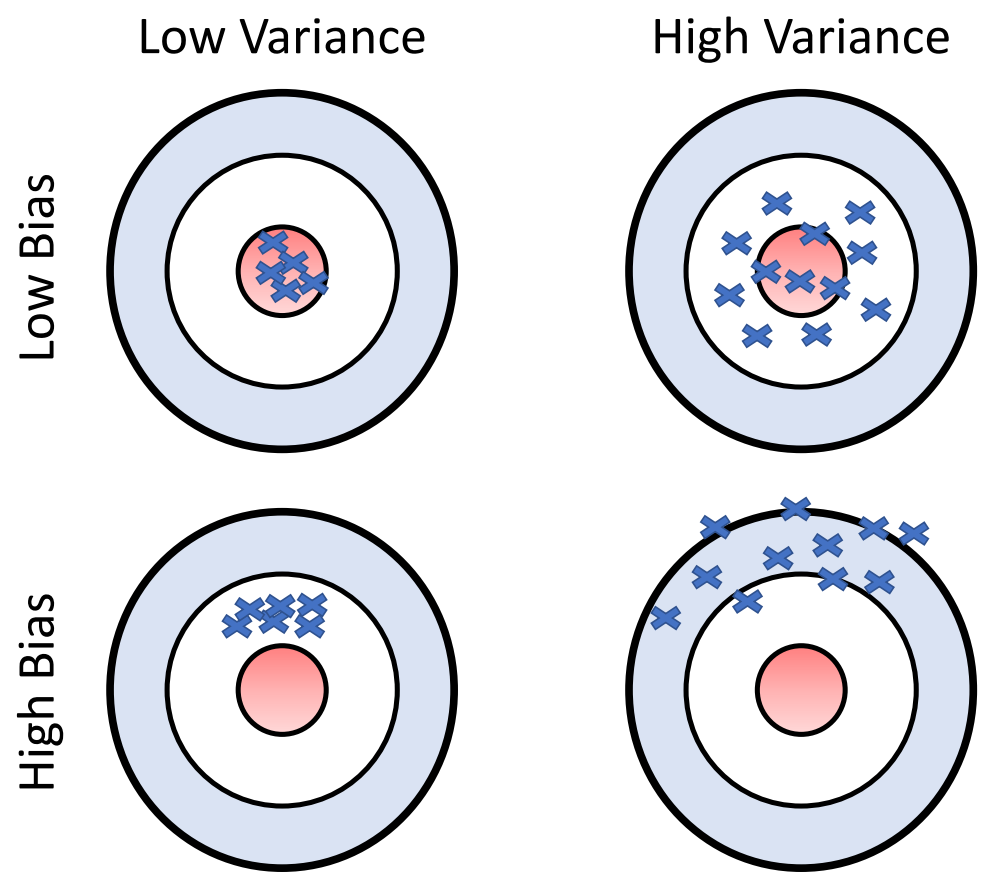

Figure 2.6: Graphical illustration of bias and variance.

There are different kinds of ensembling [44]. In what follows, we describe three main methods:

Simple averaging: This ensembling method simply takes the output average of the base models, and is one of the commonly used methods due to ease of use.

Bagging: It stands for Bootstrap Aggregation. Here we choose a random subset of training samples with replacement, which means that the subset has the same size as the original training set but some samples may appear more than once while others may not appear at all. Many such subsets are created and a regressor model is built for each subset. Finally, the results of these multiple regressors are averaged. In case of classification, we do majority voting. All the models run in parallel and do not interact with each other, and equal weights are assigned to each model as well as 
each sample. Bagging decreases error by decreasing the overall variance. The reason is that if we take the variance of the average of outputs, it will be lower than the average of the individual variances of the outputs by a factor of $1 / N$. Random forest is a famous bagging model which uses variations of multiple trees. It performs better compared to individual trees model by classifying a new object where each tree gives votes for that class and the forest chooses the classification having the most votes (over all the trees in the forest). In case of regression, it takes the average of outputs of different trees [45].

Boosting: It involves sequential learning of the models. The first model is trained on the whole dataset with all the samples. In the following iterations, the same model is trained on the subsets of training set (without replacement) based on the performance of the previous iteration [46]. The samples which are not well predicted are given higher weight in each successive round, and the weights are decreased for those who are well predicted. This means that our next model will do well for the areas where the previous model lacks performance. In Bagging, we just averaged the results of all models which run independently in parallel. Here we try to learn from the errors made in each model and incorporate this into our next model, which means we are boosting our new model. Being an iterative process, it continues to add models until a limit is reached in the number of models or accuracy. Boosting generally gives better results by reducing bias and variance, but sometimes it tends to over-fit the training data as well.

Stacking Stacking is an ensemble learning technique that combines multiple regression models via a meta-regressor. The base models are trained based on a complete training set, then the meta-model (also known as an aggregator) is trained on the outputs of the base models as features. The base level often consists of different 
learning algorithms and therefore stacking ensembles are often heterogeneous. The meta-model assigns different weights to the outputs of different base models. For a classification problems, meta-model is usually logistic regression. However, for a regression problem, various models can be used including the basic linear regression or a complex neural network [47]. This meta-model is used to combine their predictions strategically, with the aim of reducing the generalization error. This method combines the power of both, boosting and bagging, as it does not only involve different base models trained on the whole dataset, but it also assigns optimal weights to different models instead of simply averaging their outputs [48]. This is why we have chosen stacking method for ensemble learning in this work.

\subsubsection{Evaluation metrics}

To measure the accuracy of the models discussed above, we use three error metrics: the normalized mean absolute percentage error (NMAPE), the normalized root mean square error (NRMSE), and $R^{2}$ also known as the coefficient of determination (COD).

\subsubsection{NMAE}

This metric measures the accuracy by comparison between actual and predicted datasets using the absolute values of their differences as given in (2.14). In this equation, $\hat{y}_{i}$ is the predicted energy consumption and $y_{i}$ is the actual energy consumption for a given time at $i$ th sample. The lower the NMAE, the better the prediction model.

$$
\mathrm{NMAPE}=\frac{1}{N} \sum_{i=1}^{N}\left|\frac{\hat{y}_{i}-y_{i}}{y_{i}}\right| .
$$




\subsubsection{NRMSE}

The NRMSE metric is the square root of the sample standard deviation of the difference between predicted and observed values (2.15). Here, $y_{i}$ is the predicted value of the $i$ th sample and $y_{i}$ is the actual energy consumption value. This is the most commonly used error metric used in literature for time series regression problems such as electricity forecasting [49-51].

$$
\text { NRMSE }=\sqrt{\frac{1}{N} \sum_{i=1}^{N}\left(\hat{y}_{i}-y_{i}\right)^{2} .}
$$

\subsubsection{3 $\quad R^{2}$ (coefficient of determination)}

$R^{2}$ is a measure of how close the data is to the fitted regression line. If $\hat{y}_{i}$ is the difference of predicted energy consumption of the $i$ th sample from the actual energy consumption of the $i$ th sample, and $y_{i}$ is the difference of the mean energy consumption of all true values from the actual energy consumption of the $i$ th sample, then the COD is defined as $\mathrm{R}^{2}=\sum \hat{y}_{i}{ }^{2} / \sum y_{i}{ }^{2}$.

\subsubsection{Related Work on Energy Forecasting}

In recent years, a plethora of works has appeared in the literature, addressing the forecasting of energy consumption [3,52-54]. We can classify the work related to data analytics in smart grid systems into three categories (i) the first one aims at gaining a deep understanding of the intrinsic properties of energy data including the visualization and filtering (for example, trends based on temporal consumption or weather) (ii) the second category focuses on forecasting the electricity consumption for a given area and period based on previous readings (iii) and the third set looked at giving recommendations to users based on the available supply of electricity and 
its usage in the locality by considering the user's consumption behaviour.

Several authors have studied the problem of customer segmentation in the DR context. For instance, a clustering technique using k-means aiming at determining natural segmentation of customers and identifying their temporal and spatial consumption patterns has been considered in [55]. Similarly, [56] has examined the use of big data analytics to propose a scheme for the selection of right customers for a given level of enrolment in DR program. A cloud-based big data analytics framework in smart grid systems has been considered in [37] by focusing more on data visualization. The accuracy of SVR models coupled with different clustering methods including k-means, k-medoids-mean, and k-medoids-min on the existing dataset collected over a period of two years at three different locations in Japan has been investigated in [57]. Dong et al. have combined convolutional neural network with k-means clustering for short-term load forecasting and have verified their results with different error parameters including NRMSE and NMAE [58]. Furthermore, [59] proposes a pattern forecasting ensemble model with iterative prediction procedure and compares the obtained results with five forecasting models using different clustering techniques. The work proposed in [60] discusses the big data management and implementation in smart grids and focuses on the required and available hardware and software tools. In [61], authors use neural network to compare commercial and residential buildings' data. In [62], Apache Spark has been presented as a unified cluster computing platform for storing and performing big data analytics on smart grids.

Recently, artificial neural networks have been considered in [63] as a popular method for predicting electricity loads thanks to the non-linear and adaptive nature of the prediction model. Temporal patterns arising in electricity consumption timeseries data using a real-world, large-scale dataset have been explored in [64], in which 
authors proposed a novel algorithm for time series clustering based on Hausdorff distance. The proposed method scales to large datasets, and does not have to be confined to electricity consumption data. Similarly, authors in [56] proposed method scales to large datasets and is composed of a linear response modelling and a fast heuristic selection algorithm. Another similar and interesting conclusions were found in [65] where a linear programming (LP) algorithm to be integrated into the energy management system (EMS) of a household or a small business is provided. Such algorithm maximizes the consumer utility or minimizes its energy cost via bidirectional communication with the electricity supplier. The interaction takes place on an hourly basis using a rolling window algorithm to consider the energy consumption throughout the twenty four hours of the day. Bidirectional communication is a vital component of a smart grid and as such, is used to design the proposed procedure. A case study demonstrates the usefulness of the proposed algorithm to maximize the utility (or to reduce the electricity bill) of a consumer that integrates the proposed procedure in its EMS. An practical methodology that can be used to drive improvements in peak load forecasting for a power system zone was proposed in [55]. The proposed approach could inform load forecasting about individual households. Such forecasting is vital for the design of microgrids and intelligent distribution systems. The methodology suggests that different consumer classes might require different forecasting approaches. In [66], potential key enablers for the proposed collaborative edge-cloud computing framework have been identified and the associated key challenges have been presented to foster future research activities in this domain. 


\subsection{Summary}

In this Chapter, we discussed the background of IoT and how it is changing the landscape of smart grids. We then explained TGDR project and our role in it. Finally, we have explained the importance of energy analytics along with related work done in this domain. In the next two chapters, we provide our contributions using the above background to implement smart grid data communication and perform data analytics. 


\section{Chapter 3}

\section{Implementation of the Smart Grid Communication System}

\subsection{Introduction}

In this chapter, we give the details behind the deployment of our IEEE 2030.5-based communication system in real environments and present our testing results. This chapter is organized as follows. Section 3.2 details the proposed communication system architecture including the message format, topology, and security requirements. Section 3.3 provides detailed results and discussions on the deployment and testing of this system in three real-world sceneries (i) Carleton University, (ii) Sandcherry, and (iii) Viewmount. Section 3.4 concludes this Chapter.

\subsection{System Model}

The considered system model is presented in Fig 3.1. The collected data is sent every 15 minutes from homes to the utility. Then, recommendations are communicated back from the utility to homes via the mesh network. 


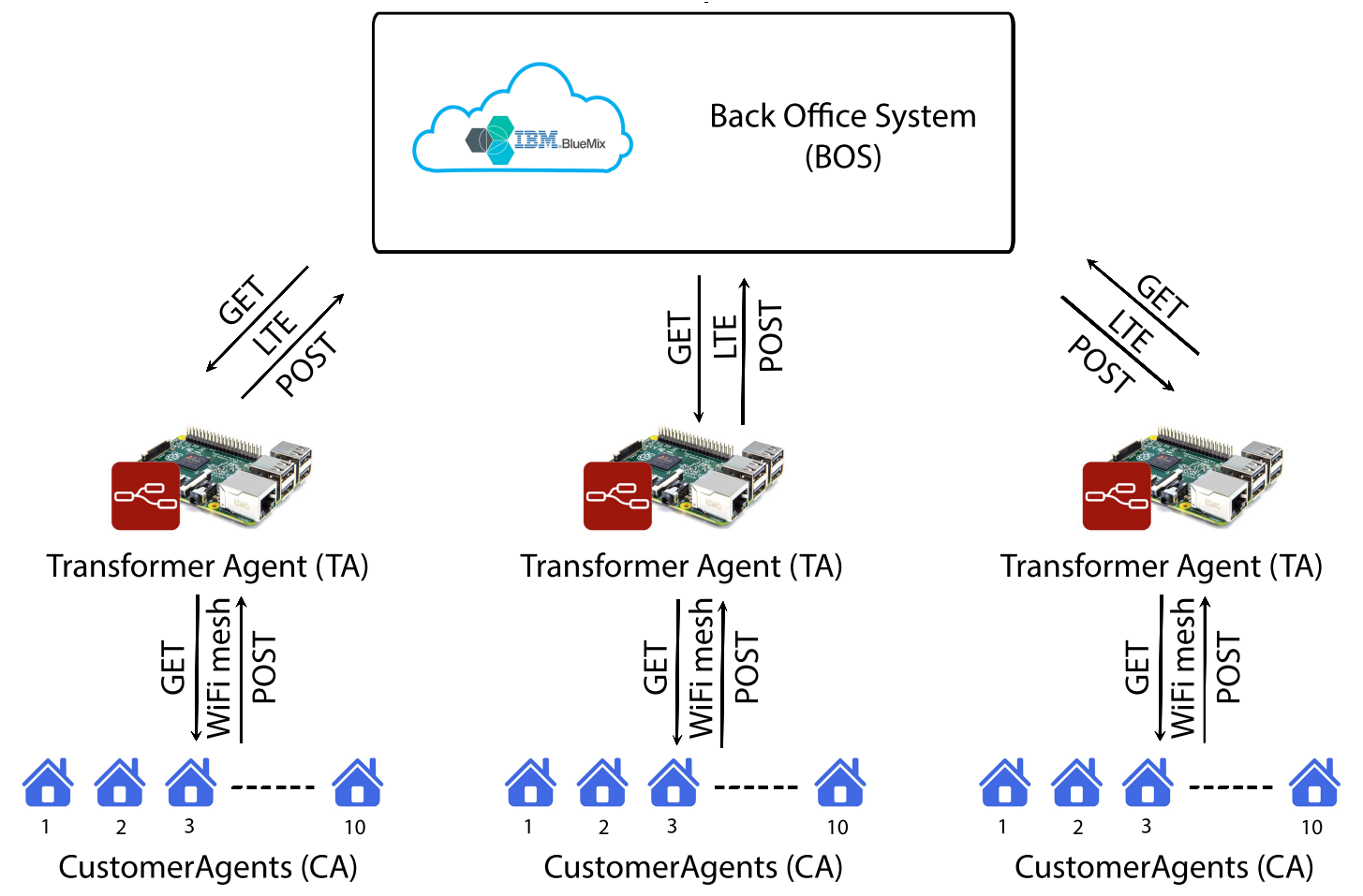

Figure 3.1: System model for smart grid communication system.

The main system components including the BOS, the TA, the CAs, and the HEMS controller are defined in what follows:

1. Back office system: is a cloud-based software system that provides system visibility and control to the utility. The BOS manage the fleet of TGDR elements, exchanges transactional demand response messaging and commands, monitors and records transactional demand response activities.

2. Transformer agent: is physically located adjacent to the neighbourhood distribution transformer. It is located in an exposed location and therefore must handle environmental extremes. The TA monitors the local Grid at the lowvoltage side of the transformer and communicates with the CA element to coordinate DR actions. The TA is also in communication with the utility through the BOS for command, control and monitoring of the system. 
3. Customer agent: is physically located at the customer's premise near the service entrance, meter, and breaker panel. It is a trusted piece of distributed system operator (DSO) equipment they administer. The CA is capable of performing local power measurements for validating TDR action. The CA communicates with the TA to coordinate transactional demand response actions, and exposes a secure, open architecture interface to the respective prosumer's HEMS so that prosumer and DSO privacy plus cybersecurity are preserved.

4. HEMS controller (HEMSC): The HEMSC is physically located at the customer's premise and is responsible for integrating intelligent monitoring and control of a wide variety of customer-premise Behind-the-Meter technologies, or devices (appliances, generators, load controllers, thermostats, ... ). It hides the diversity of controlled devices plus the complexity of the device activity from the Grid i.e., the CA, TA, and BOS. It is also implemented using an open and standards-compliant interface to the DSO through the CA, TA, and BOS. The HEMSC translates the information from the CA into protocol formats and commands that the particular technology-specific device controller requires meeting the power demand quota which is sent from TA to each CA.

\subsubsection{Communication between CAs and TA}

In this system, a reliable mesh network is established between the homes with an aggregating node as the electric transformer in the neighbourhood. It has a TA attached to it, whereas houses in the neighbourhood have CAs attached to them. The TA and CAs perform data processing and transmission for the nodes in the network. The messages are communicated via multi-hops in XML format in compliance with IEEE 2030.5 profile [14] and other requirements detailed in Section 3.2.3. The Wi-Fi 
based mesh network is used between CAs leveraging the OLSR routing protocol while LTE is used between TAs and the main utility for secure wide-range communication. The data communicated between the homes and utility is coming from HEMS. This data is obfuscated data to maintain customer privacy.

\subsubsection{Communication between TAs and Cloud}

In addition to the TA negotiations with various CAs, there is another level of negotiations as well. These are performed among the TAs by the utility. Therefore, we send all the data collected at the TA in real time to the BOS (cloud) for storage and processing. LTE/cellular networks are used here for this communication because the TA is located at the pole along with transformer where there is no Wi-Fi. We use 4G/LTE Base Shield by Sixfab with Quectel EC25 4G/LTE Module for ultra-fast cellular Internet communication. Their specifications are detailed in Section 3.2.4. Once data arrives at the cloud, we store it on IBM Cloud's Cloudant NoSQL database in JSON format. Additionally, the data communicated between CAs, TA, and the BOS is secured by TLS 1.2 and is pre-processed and aggregated for privacy, better scalability, and to reduce the traffic. We then perform descriptive and predictive analysis of the data using various prediction techniques as presented in Chapter 4.

\subsubsection{System Compliance with IEEE 2030.5 Standard}

\subsubsection{Topology and routing protocol}

One of the main challenges facing the proposed system architecture is the covered range. In case of star topology, the access point (the TA) would have required a powerful antenna to cover the whole neighbourhood zone. Therefore, a Mesh network architecture was chosen to implement the smart grid communications solution $[67,68]$. 
This decentralized type of wireless networks does not rely on any pre-existing infrastructure to coordinate the communication between the clients (CAs) and gateway (TA), rather it establishes peer-to-peer connections [69]. Each CA in the network is connected to adjacent $\mathrm{CA}$ and participates in routing by forwarding data to neighbouring CAs as shown in Fig 3.2. The determination of which nodes forward data is made dynamically based on network connectivity strength between them and is governed by OLSR routing protocol explained later in this section. Moreover, this decentralized communication model allows for a smoother self-healing network process. In case one node drops for whatever reason, communication is re-routed through a different node.

In this work, we use OLSR as a routing protocol. Despite that our system does not have mobility for CAs, we still need the fast convergence offered by OLSR. By installing OLSR protocol on all CAs and TAs, and by setting them up in ad-hoc mode, we were able to connect them through Wi-Fi in a mesh network.

\subsubsection{Restful communication}

The Smart Energy Profile (SEP) 2.0 is designed to implement a REST architecture. It is built around the core actions of GET, HEAD, PUT, POST, and DELETE. Any application protocol that can implement a RESTful command set could likely be used with SEP 2.0, but HTTP is a required baseline for interoperable implementations [14]. Moreover, it is one of the most popular protocols and is very familiar to consumers and developers. Therefore, we have used RESTful communication between TAs and CAs, and CAs and HEMS to get and post messages. Any node can be a server and a client at the same time for each other because it is a two-way communication to exchange the function sets. For example, while pushing data from home to cloud, CA 


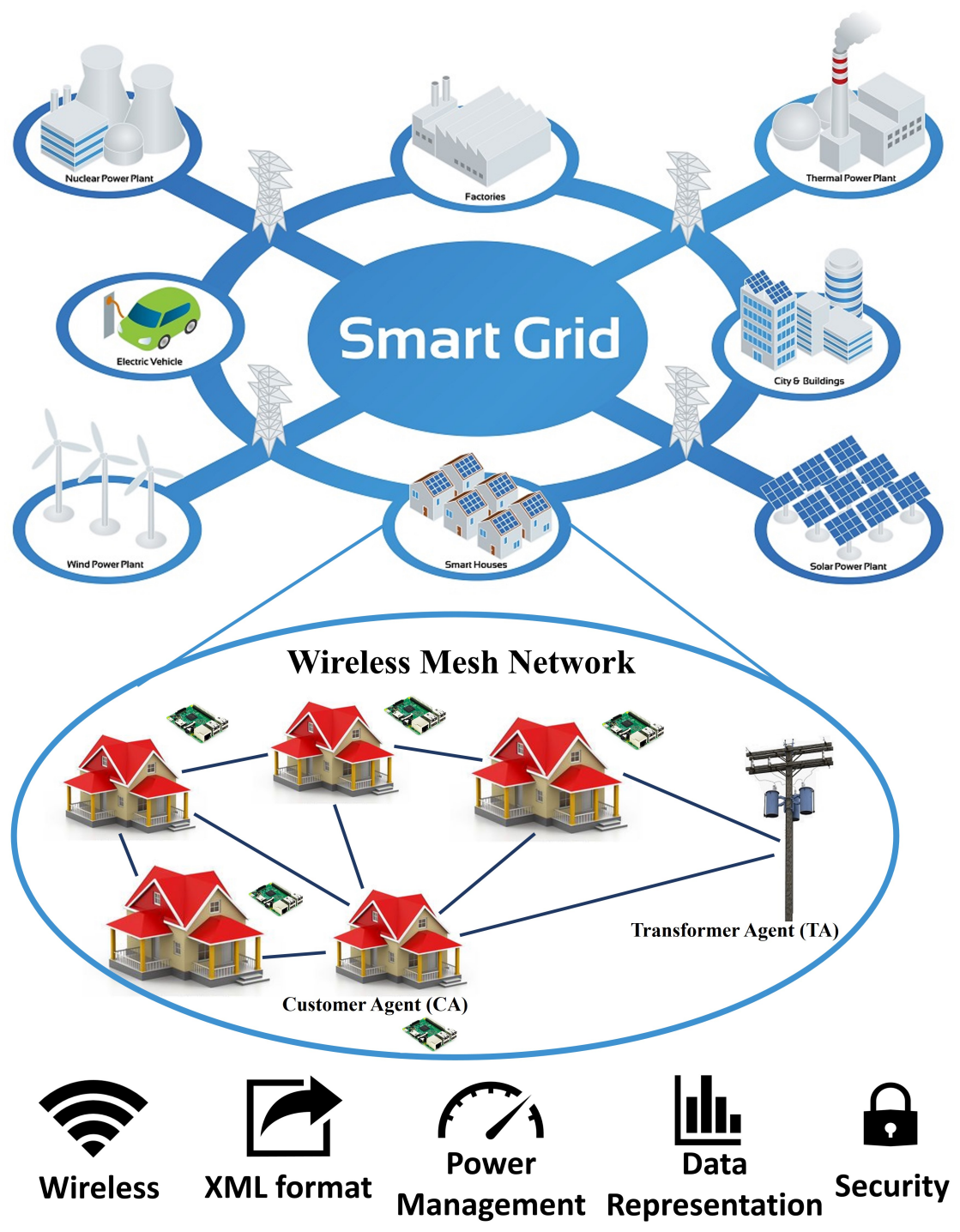

Figure 3.2: Multi-hop mesh network between the homes and the local transformer.

acts as a server for the TA, but while pushing information back from cloud to CA, the CA acts as a client for the server TA. In summary, we can think of TA and CA devices as very light-weight little web servers and clients interfacing with each other in a RESTful manner. Although there is HTTP 2.0 introduced but is not yet a part of IEEE 2030.5 standard, so we use HTTP 1.1. 


\subsubsection{Message format and function sets}

As per the IEEE 2030.5 standard, the format of the communicated files is XML and EXI. As we mention earlier, TAs and CAs are servers and clients for each other to develop a RESTful communication. Servers have to use both the formats while clients can use either of them. This is used to communicate energy data and action requests. The XML version used is 1.0 and UTF-8 encoding for XML payloads. The advantage of the XML format is that it is both human and machine-readable and is widely used in IoT applications, therefore we do not run into interoperability issues. The W3C Efficient XML Interchange (EXI) is data storage and transfer format that has the mechanics of XML, but it is more compact, faster to exchange, and faster to process [70]. The exchanged messages have different function sets as part of SEP 2.0 schema, some of which are mentioned below:

- Demand Response and Load Control

- Load Shed availability

- Energy Usage Information

- Predicted Consumption

- Green Energy Generated

- Consumption Reduction Negotiation

- Price Communication

Here the negotiation parameter is controlled by the consumers and they decide on how much in terms of percentage they want to negotiate with the utility at certain times of the day. Each function set (for example, demand response load control and metering/energy usage) is independent and may or may not be implemented by 
the utility. For example, metering/energy usage function sets do not require to be communicated in real-time as per the SEP 2.0 standard [14]. Details of the algorithms using and optimizing these function sets are beyond the scope of this research.

\subsubsection{Security measures}

Security is very critical to systems like smart grid. The 2015 Global State of Information Security Survey reported that power companies and utilities around the world expressed a six-fold increase in the number of detected cyber incidents over the previous year. Survey further states that 79 incidents were reported to Industrial Control Systems Cyber Emergency Response Team (ICS-CERT) in 2014, and 46 incidents in 2015 [71]. Therefore, instead of adding security as a non-functional feature, it was important to start with security in mind as a vital part of this work.

Network authentication: Furthermore, to protect the communication network, certain configurations were set, such as assigning unique SSIDs, static IP addresses and password. Mesh network is created after all devices boot up. The Raspberry Pi's constantly look for matched Mesh network with their static IP addresses. The TA device establishes a mesh network under a certain SSID and omits non-authorized IP addresses and closes all non-random ports to ensure that only certain CAs are authorized to join the Mesh network and can communicate with other nodes in the network - this prevents unauthorized entities from joining the network.

\section{Encryption using Ron Rivest, Adi Shamir and Leonard Adleman (RSA)}

keys: A remote access feature is added to allow secure remote access using the Secure Shell protocol (SSH). While it is important to secure the end-points, in this case the CAs and TA's, it is extremely important to secure the data flowing on the network, too. This is done by applying encryption. We created a customized 
version of an open source code that uses asymmetric encryption and changed it from using symmetric CAST5 with 64-bits block and 40-128 key size to use the strong asymmetric RSA encryption with a key size of 2048. The RSA keys used in this project are 2048 bits and contain a user-name and pass-phrase. They were generated at the nodes and then the public keys of CAs were manually inserted into the TA, while the TA public key was manually inserted into all CAs. We encrypt all the XML files using that keys before communicating happens. Larger key size generally means more secure encryption. However, there is a trade-off between key size and encryption/decryption time. The larger the key size the longer the decryption time as shown in Fig 3.3. Although the time depends on a number of factors including the machine's specifications, but generally, every doubling of the RSA key length decreases the decryption speed by 5.9 times and encryption speed by 3.2 times [72]. we can reduce this overhead by using symmetric keys along with asymmetric keys as a part of future work.

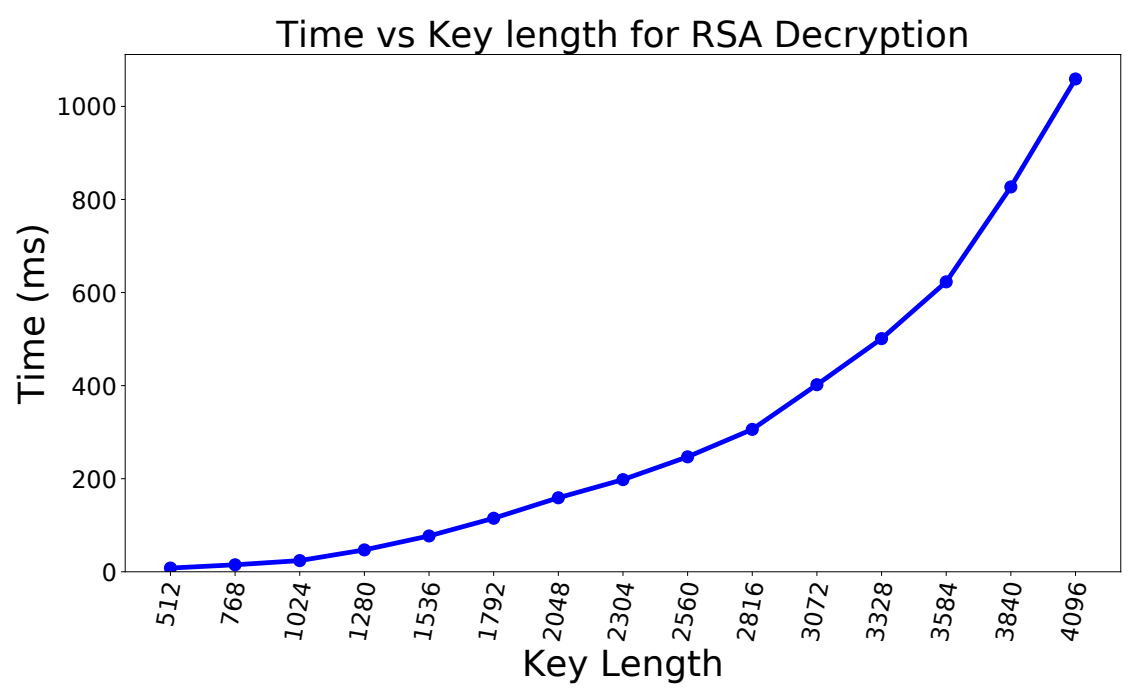

Figure 3.3: Decryption time vs key length. 
Encryption using TLS 1.2: The communication of encrypted files between clients and servers is enhanced using HTTP over TLS [73] (also known as HTTPS) using TLS version 1.2 [74]. This is the same high-level security used in Internet banking and is well understood and studied by security experts. The TLS handshake mechanism provides mutual authentication based on secure certificates installed at the Raspberry Pi. Communication between TA and utility BOS is also secured using HTTPS to ensure end-to-end security. Another reason to use HTTPS is that it meets US National Institute of Standards and Technology (NIST) standards (Suite B) [75] and IEEE 2030.5 requirements.

\subsubsection{Safety and physical protection}

Devices and users' safety is given paramount importance since the initiative of the TGDR project. To protect our nodes from extreme weather conditions $\left(-40^{\circ} \mathrm{C}\right.$ to $+85^{\circ} \mathrm{C}$ ) and thefts, we used NEMA Type $4 \mathrm{X}$ enclosure [76] along with fan and heater according to the IEEE 2030.5 standard. The TA is mounted on the pole with a solid mechanical attachment designed by CIMA Inc. All devices connecting at or within the customer premise are meeting the Ontario Electrical Safety Code rules.

\subsubsection{Selected Hardware and Implementation Tools}

TA selection: In our system, CAs and TAs are Raspberry Pi 3 modules. Initially we used a modified version of the open source project code Byzantium [77] which was responsible for creating and maintaining OSLR based routing mesh network between all the CAs connected. An Intel-based board was required at the TA to run Byzantium, so we used a laptop as TA initially. Intel Edison board was later

considered due to portability issues but we soon ran into compatibility issues with the Linux versions and decided to opt for Raspberry Pi as TA. We installed OLSR routing 
protocol on R-Pi and created a mesh networking. For this purpose, we improved and customized the code of open source available High Speed Multimedia Mesh for Raspberry Pi (HSMM). We then needed strong TA for edge analytics so we considered opting for tinker board which is more powerful in terms of processing. It has same operating system and baseboard as RPi but different specs detailed later in this section. But soon we started experiencing various issues with its kernel, for example, no connectivity support for external USB WiFi dongle, and forcing connectivity to first connected SSID even though it is not in range. We tried changing the kernel but due to limited time and online community support (as its a new product), we had to revert back to Raspberry Pi.

Most of our system is programmed in Python and we used Node-Red (JavaScript) to program the communication between TA and Cloud. Hardware Specifications of some of the components used in our project are described below:

LTE shield: This acts as an interface bridge between Mini PCIe 3G/4G/LTE modules and Raspberry Pi. It supports Micro SIM Card socket and is powered from an external $5 \mathrm{~V}$ source. Its working temperature range: $-40^{\circ} \mathrm{C}$ to $80^{\circ} \mathrm{C}$. It is mainly used for video streaming or high-speed data transferring. The connection types are: USB and UART [78].

LTE module: Quectel EC25 Mini PCIe [79] is especially for M2M and IoT applications and delivers $150 \mathrm{Mbps}$ downlink and 50Mbps uplink data rates. The module is backward-compatible with existing EDGE and GSM/GPRS networks, ensuring that it can be connected even in remote areas devoid of $4 \mathrm{G}$ or $3 \mathrm{G}$ coverage. Following are its key features:

- Bandwidth: 1.4/3/5/10/15/20MHz 
- Temperature Range: $-40^{\circ} \mathrm{C}$ to $+80^{\circ} \mathrm{C}$.

- Supply Voltage: 3.3V.

- Data Speeds:

LTE FDD: Max 150Mbps (DL)/Max 50Mbps (UL).

LTE TDD: Max 130Mbps (DL)/Max 35Mbps (UL).

- Output Power: Class $3(23 \mathrm{dBm} \pm 2 \mathrm{~dB})$.

- Power Consumption: $3.6 \mathrm{~mA}$ at sleep mode and $35 \mathrm{~mA}$ at idle mode.

LTE antenna: The EC25 module includes a main antenna interface, an Rxdiversity antenna interface which is used to resist the fall of signals caused by highspeed movement and multipath effect, and a GNSS antenna interface. The impedance of the antenna port is $50 \Omega$. However, we due to outdoor deployment and severe weather conditions, we decided to use the Proxicast 4G/LTE CrossPolarized 7-10 dBi Fixed-Mount Panel Antenna [80]. It works for frequencies 698-960 MHz and 1710-2170 MHz. We have $2100 \mathrm{MHz}$ LTE band at our deployment sites as well as Carleton University so this weatherproof antenna served all our requirements.

\subsection{Site Testing Results and Discussions}

We generated XML files following the requirements detailed in Section 3.2.3 in order to simulate a real life scenario where several CAs send their information to the TA. Data collected at multiple TAs is finally sent through LTE to IBM Cloud for storage and processing. Several tests were conducted to prove the functionality and scalability of our solution. We deployed it across three different real-world environments during testing. Three different environments are chosen because each has different specifics, 
for example, buildings have different architecture and map, they have different material of walls, and different surroundings for interferences. Initially, we performed several tests at Carleton University in different configurations to make sure our hardware and software are working perfectly according to the requirements mentioned in Section 3.2.3. Next, we deploy it at two sites chosen by Hydro Ottawa Limited for TGDR project. Residents of those homes have participated in TGDR project. The two sites are located in Sandcherry and Viewmount locations of Ottawa city. All the necessary approvals were taken by Hydro Ottawa Limited and City of Ottawa to enter the premises. All the ethics, protocols and standard operating procedures at the sites were fully observed by us during this research. In the following subsections, we explain the results of communication for all these sites.

\subsubsection{Carleton University}

Initial testing was conducted at Carleton University campus for verification of our communication system including codes and nodes. These included various tests including scalability and reliability. XML files were communicated in a RESTfull manner with all security features enabled, and according to the requirements as detailed in Section 3.2.3. A part of these findings has been published in [81].

We divide our testing process into multiple steps, which are summarized as follows:

1. Check the basic connectivity between two nodes using PING command.

2. Create XML files based on message format as described in Section 3.2.3.3 and communicate them using SSH and secure copy. The file is shown in Fig 3.4(a).

3. Encrypt the files using RSA keys and communicate them between two nodes to make sure that our RSA based encryption is working as described in Section 


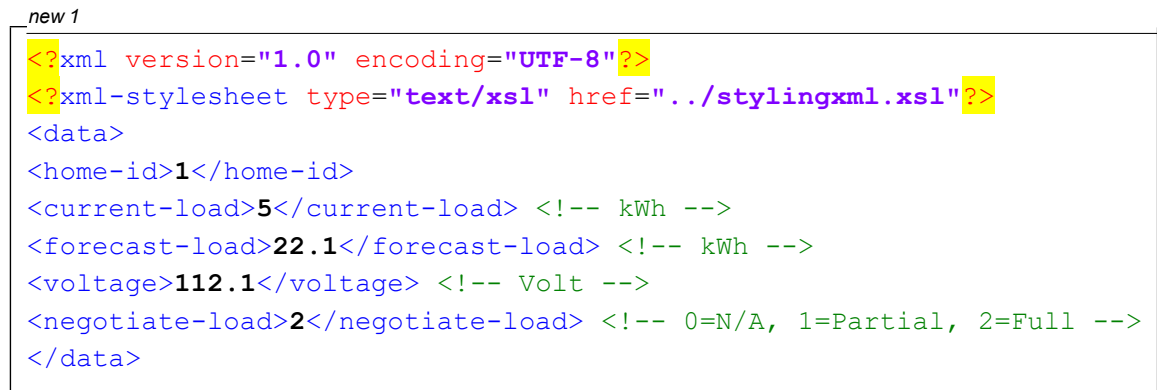

November 28, 2018 2:4

(a) Original XML file.

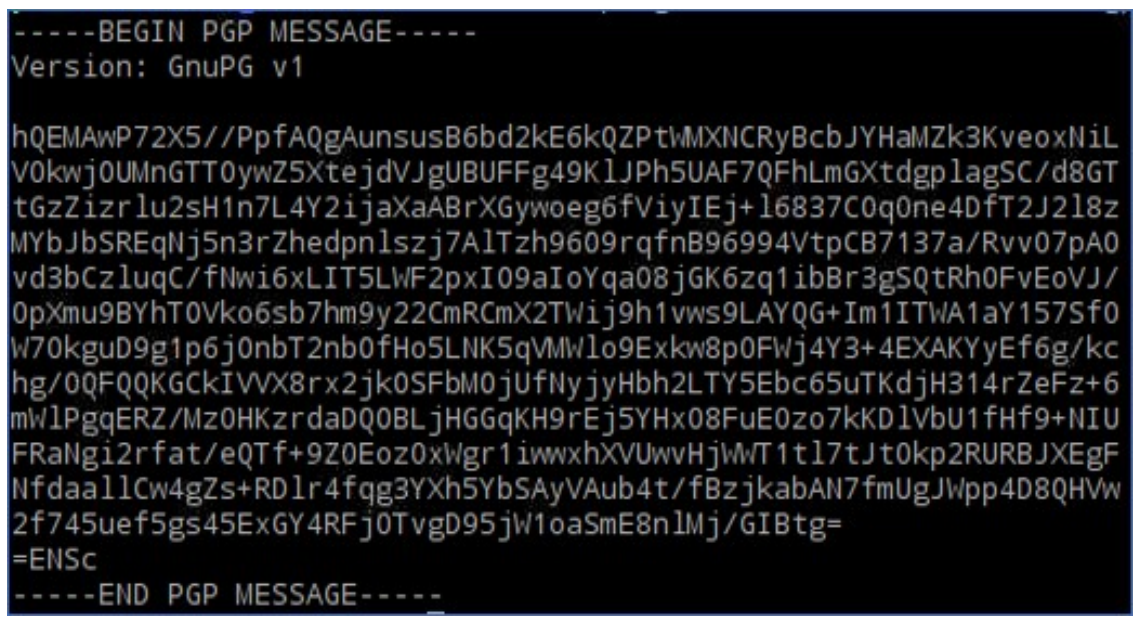

(b) Encrypted XML File.

Figure 3.4: Original and encrypted XML files.

\subsubsection{This is shown in Fig 3.4(b).}

4. Create our nodes as RESTful web servers and clients by installing, and test the GET and POST queries using CURL commands as described in Section 3.2.3.2.

5. Wrap our HTTP communication with TLS 1.2 security layer and test our communication that is now encrypted using HTTPS as shown in Fig 3.5.

6. Add multiple nodes to our network and perform tests for scalability, distances, latency, multi-hopping, reliability and simulating a node dropping under different deployment scenarios. $-1-$ 


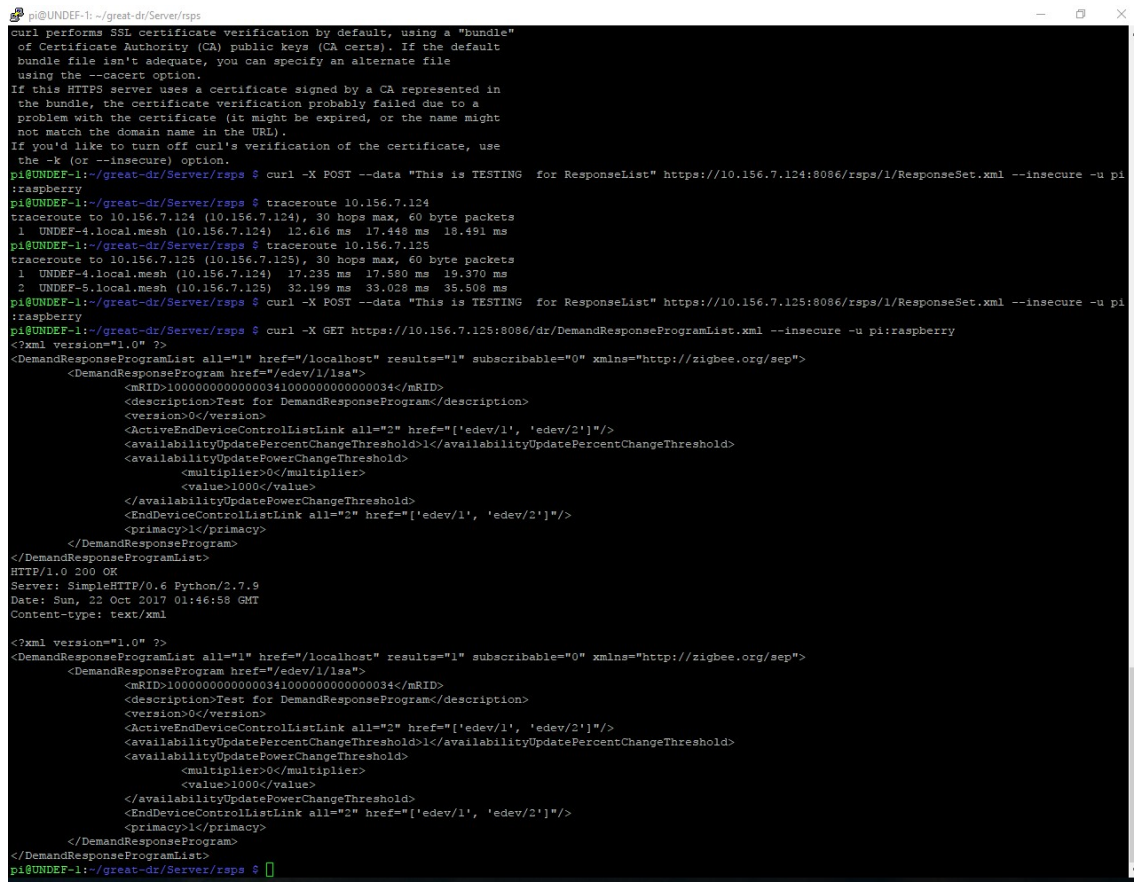

Figure 3.5: HTTPS communication between two nodes.

7. After successful communication between the CAs and TA, we test our TA to BOS communications.

\subsubsection{Test-1: Initial setup of mesh network within the lab}

The first test was performed within the IoT Lab at Carleton University with three nodes placed at distant points in the same room with a line of sight. Ad-hoc mode was set up on all the nodes and OLSR protocol was installed. All the nodes were connected to the same channel $(2.412 \mathrm{GHz})$ and were successfully able to ping each other at default parameters without packet loss. Two of them were set up as an internal node of the mesh network (CAs), while the third node (TA) was set up as a gateway node. We then exchanged XML files through SCP at the rate of $413 \mathrm{kbps}$. Each file size was $2 \mathrm{~kb}$, so it took about $5 \mathrm{~ms}$ for a file to be transferred. We then exchanged the encrypted files using 2048-bit RSA encryption, which added an additional $159 \mathrm{~ms}$ for decryption and $9 \mathrm{~ms}$ for encryption. This shows that decryption is approximately 


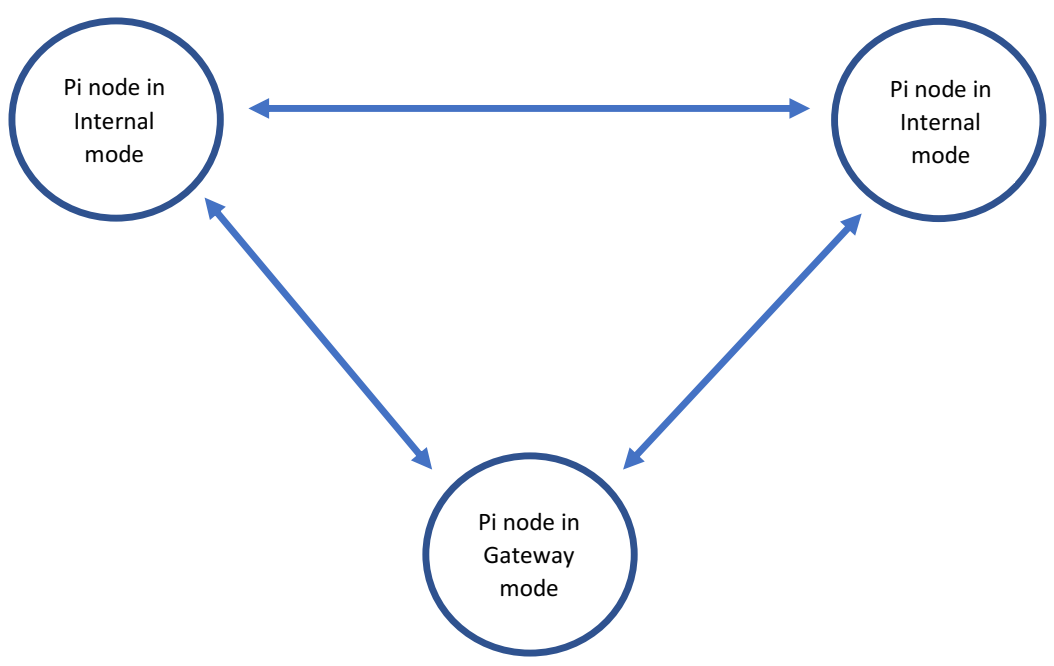

Figure 3.6: Initial setup for Test-1.

18 times slower than encryption. Further, we installed a RESTful server on all the nodes, and set the CAs as clients and TA as a server. We successfully queried GET and POST commands for all four nodes using the same XML file. Finally, we added a TLS 1.2 security layer, and installed certificates and successfully 'posted' data from TA to all the CAs individually, and 'got' from all the CAs to TA [4]. The one way trip time was less than one second for each query.

This test successfully completed steps 1 to 5 of the testing process as described in the previous subsection. The remaining steps will be explained in the following subsections. The overall setup for this initial testing is shown in Fig 3.6. This was the basic testing with all the nodes laying in ideal conditions i.e. line of sight and in close proximity to each other without any disturbance, for example, node going off etc. After successfully testing all the IEEE 2030.5 requirements in basic network configuration, we then test the network performance in case of complex scenarios including obstacles. 


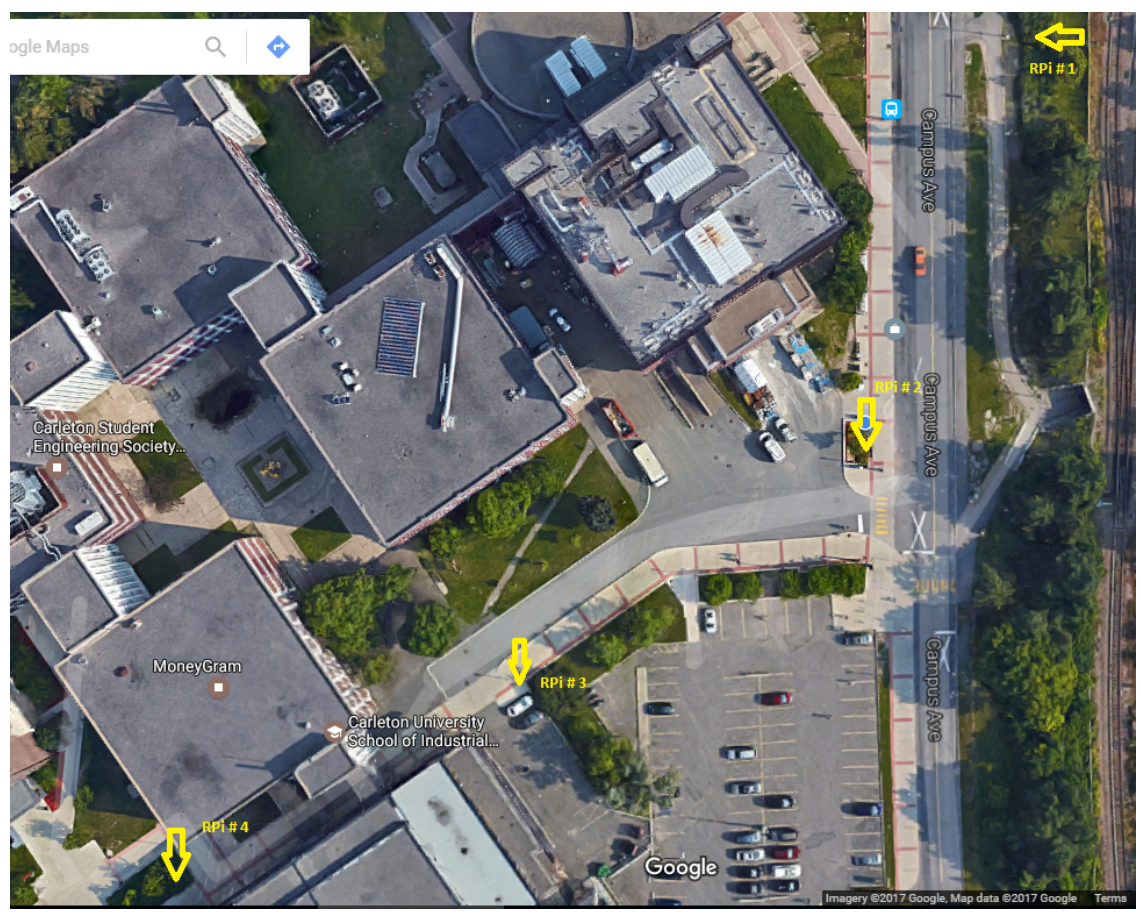

Figure 3.7: Placement of nodes for Test-2.

\subsubsection{Test-2: Maximum distance for line of sight}

In this test, we bring the nodes to open area in Carleton University. The objective is to test the maximum distance for which one node is able to ping the other in Line of Sight. Refer to Fig 3.7 for the position of the nodes. We found this distance to be $65 \mathrm{~m}$ between RPi-1 and RPi-2. We then placed two more RPis, each within the line of sight of the previous RPi such as to extend the range of our communication system. With this configuration, we were able to successfully ping from RPi-1to RPi4, covering a total distance of around $240 \mathrm{~m}$. This also confirms our mesh network successfully running because without the presence of RPi-2 and RPi-3, RPi-1 couldn't reach $\mathrm{RPi}-4$. We used default ping parameters for this test. 


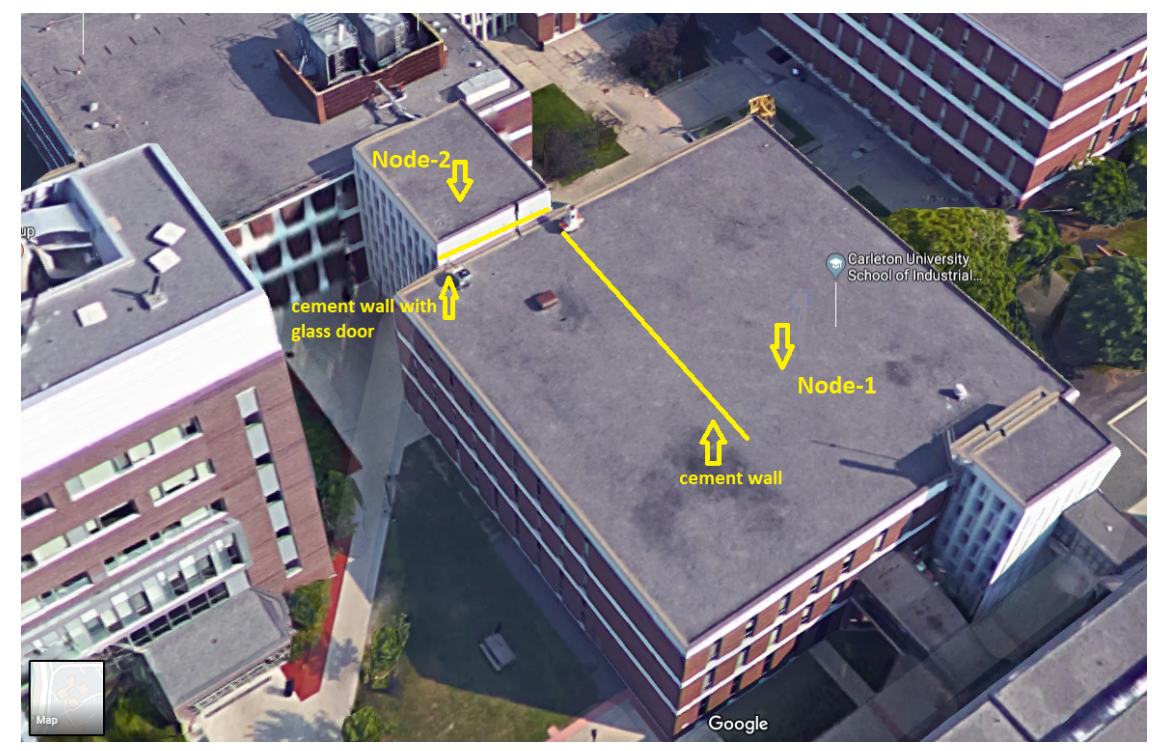

Figure 3.8: Placement of nodes for Test-3.

\subsubsection{Test-3: Nodes with obstacles}

So far we tested our nodes only in the line of sight, but in a real world scenario, there can be many obstacles including walls, doors, trees, metallic objects, electric cables etc. Therefore, we positioned our nodes with obstacles as shown in Fig 3.8 with obstacles such as the cement wall and glass door. We observe that the maximum range has been dropped considerably from $62 \mathrm{~m}$ in line of sight to just $27.5 \mathrm{~m}$ in this case. The possible solution to increase the range covered was to use external Wi-Fi dongle or antennas instead of relying on Internal antennas - this is explained in the following subsections.

\subsubsection{Test-4: Antenna}

The USB Wi-Fi dongle was initially tested but it was not so useful as it only increased the range by a $17 \mathrm{~m}$ in the line of sight. Therefore, we decided to go for external antennas. The main challenge was that only a few antennae in the market were found 
to be compatible with Raspberry-Pi kernel such that they can be set up in ad-hoc mode. We decided to test omnidirectional antenna with specifications described in Section 3.2.4. Moreover, harsh weather conditions of Ottawa was another challenge for us, due to which we considered the use of an enclosure for the Antenna. This is explained in the next paragraph.

Enclosure for antenna: Initially, we decided to use NEMA Type-6 enclosure [82]. Our testing with metal enclosures concluded that each enclosure around an antenna attenuates the signal by $90 \%$, or $10 \mathrm{dBm}$, which is considered a very high loss. Therefore, we decided to switch to non-metal NEMA Type-4 enclosures.

Antenna Orientation: Our antenna is omnidirectional which means that it radiates equal power in all directions perpendicular to an axis (azimuthal directions), with power varying with angle to the axis (elevation angle), declining to zero on the axis [83]. Its donut pattern is shown in Fig 3.9 with red showing the most power and green showing the least power. We tested our node to node communication for different antenna orientations by placing two nodes in line of sight. We first tested with their antenna parallel to each other and then repeated the test by placing their antennas perpendicular to each other, keeping all the external conditions same. We observed that there was a loss of $12 \mathrm{dBm}$ when we changed the direction of antenna.

\subsubsection{Test-5: Network performance - with vs without antenna}

After deciding on the antenna and successfully testing our basic configurations, we will now study the network performance in detail for antenna vs without antenna, with particular reference to Packet Loss, Latency and Signal Strength. The parameters used for this test are described in Table 3.1, and the placement of nodes is shown 


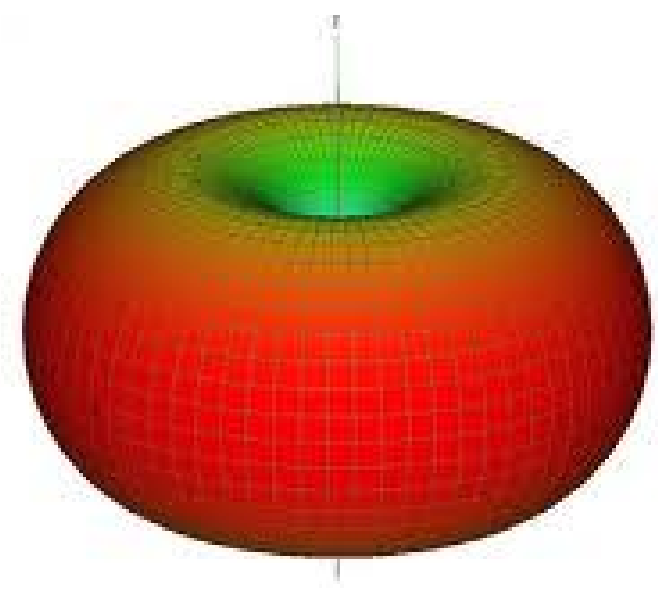

Figure 3.9: Omni-directional antenna pattern [84].

in Fig. 3.10. This area is located between University Center and Canal Building of Carleton University. All the nodes were in connected in a mesh network, and were located at $50 \mathrm{~cm}$ above the ground to simulate the real conditions. We chose the packet size to be $5 \mathrm{~kb}$, which is much larger than the actual XML file we are communicating. This is done to test the worst case scenario, for example, in case multiple CAs are sending data at the same time to the TA through the same CA in a mesh network. Number of Packets was chosen to be 100, which means we calculate the network performance 100 times and then take their average value. This helps to remove noise, if any.

Table 3.1: Parameters for Test-5.

\begin{tabular}{|c|c|}
\hline Parameter & Value \\
\hline Packet size & 5000 bytes packet +8 bytes header \\
\hline Packet sending interval & $0.5 \mathrm{sec}$ \\
\hline Number of Packets & 100 \\
\hline
\end{tabular}




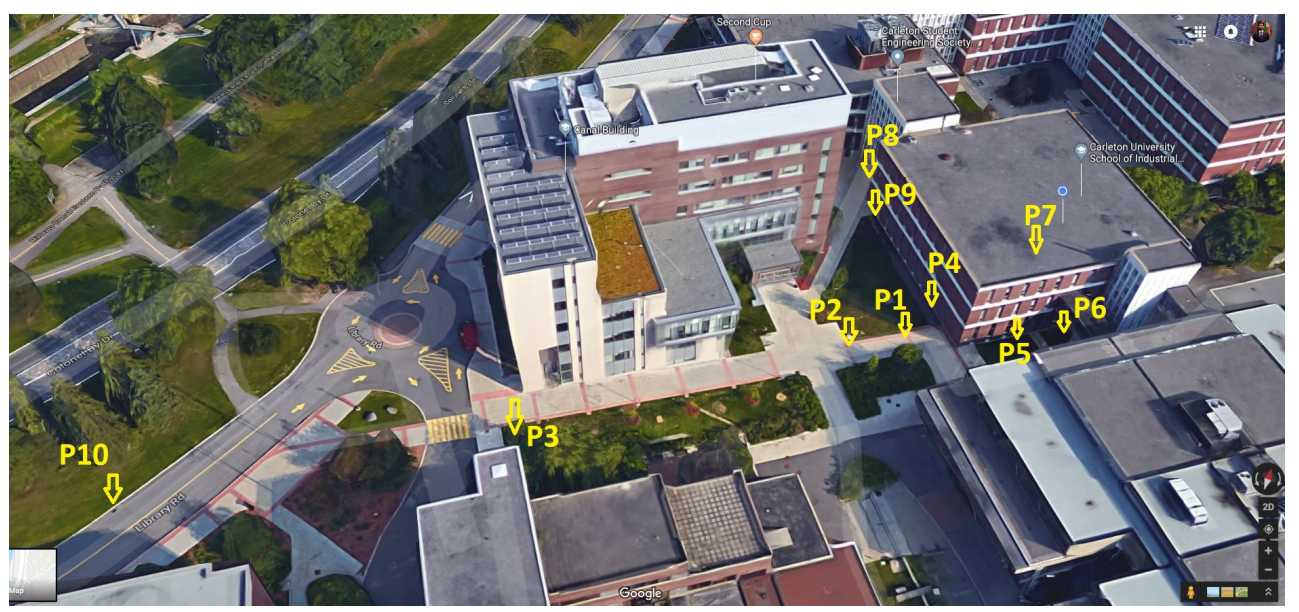

Figure 3.10: Placement of nodes for Test-5 and Test-6.

Line of Sight: In this test, we activate nodes at positions $P 1, P 3$ and $P 10$ (two at a time to avoid interference), while others are deactivated. All these nodes are in line of sight. Table 3.2 shows the network performance variations with distance for direct line of sight. The blank cells in the table mean the communication has failed. We have set a benchmark of $-60 \mathrm{dBm}$ as sufficient signal strength for stable communication in this thesis.

Table 3.2: Network performance for Test-5: Line of sight.

\begin{tabular}{|c|c|c|c|c|c|c|}
\hline \multirow{2}{*}{ Distance (m) } & \multicolumn{2}{|c|}{ Signal Strength (dBm) } & \multicolumn{2}{c|}{ Round Trip Time (ms) } & \multicolumn{2}{c|}{ Packet Loss (\%) } \\
\cline { 2 - 7 } & With Antenna & Without Antenna & With Antenna & Without Antenna & With Antenna & Without Antenna \\
\hline 9.6 & -26 & -56 & 16 & 50 & 0 & 3 \\
\hline 61.3 & -50 & -72 & 18 & 7055 & 1 & 34 \\
\hline 141.8 & -60 & - & 176 & - & 12 & - \\
\hline
\end{tabular}

We observe significant performance improvements in terms of latency, packet loss, and signal strength when using an external antenna. For example, signal strength at a distance of $61.3 \mathrm{~m}$ is 128 times better with antenna than without antenna. Moreover, at a distance of $141.8 \mathrm{~m}$, there is no signal if we do not use external antenna, however, we get a good and reliable strength of $-60 \mathrm{dBm}$ with antenna. In fact, the reason for choosing $141.8 \mathrm{~m}$ value was that it was the maximum distance where we received 
sufficient signal strength using antenna. From Test-2, we remember that $65 \mathrm{~m}$ was the maximum distance with internal antenna for the weakest signal.

Table 3.3: Cases for Test-5: No line of sight.

\begin{tabular}{|c|c|c|}
\hline Case & Placement & Distance $(\mathbf{m})$ \\
\hline A & $P 1$ and $P 5$ separated by two concrete walls, where $P 1$ has an open area beside it & 17 \\
\hline B & $P 4$ and $P 5$ separated by two concrete walls, where $P 4$ is attached beside the wall & 15 \\
\hline C & $P 1$ and $P 7$ separated by two concrete walls, including a mechanical lab & 21 \\
\hline
\end{tabular}

No Line of Sight: After testing for the line of sight, we studied our network performance with by separating the nodes with concrete obstacles as in the real world. In this test, we use nodes at Position $P 1, P 4, P 5, P 7$ and $P 8$, such that only two of them are activated at a time to avoid any interference. This test is divided into four different cases as described in Table 3.3, based on the strength of separation between the nodes. Table. 3.4 shows the network performance for each of these cases. Here the blank cells mean communication is failed. We see from the results

Table 3.4: Network performance for Test-5: No line of sight.

\begin{tabular}{|c|c|c|c|c|c|c|}
\hline \multirow{2}{*}{ Case } & \multicolumn{2}{|c|}{ Signal Strength (dBm) } & \multicolumn{2}{c|}{ Round Trip Time (ms) } & \multicolumn{2}{c|}{ Packet Loss (\%) } \\
\cline { 2 - 7 } & With Antenna & Without Antenna & With Antenna & Without Antenna & With Antenna & Without Antenna \\
\hline A & -52 & -77 & 35 & 14302 & 0 & 97 \\
\hline B & -62 & -79 & 236 & - & 18 & - \\
\hline C & - & - & - & - & - & - \\
\hline
\end{tabular}

that there is no communication happening between nodes at $P 1$ and $P 7$ in Case- $C$. This can be explained by the presence of three concrete walls. Moreover, the heavy metallic equipment also contributed to the lack of communication. For the Case- $A$, we see a very good communication when external antenna is used, i.e., there is no packet lost, however, $97 \%$ of packets are lost if we rely only on internal antenna. This means almost no communication is happening. This further strengths the need 
Table 3.5: Network performance for Test-6.

\begin{tabular}{|c|c|c|c|c|}
\hline Node Positions & Distance $(\mathbf{m})$ & Line of Sight & Signal Strength $(\mathbf{d B m})$ & Packet Loss (\%) \\
\hline P3-P2 & 51 & Yes & -50 & 1 \\
\hline P3-P1 & 61 & Yes & -52 & 1 \\
\hline P3-P9 & 74 & No - 2 buildings as obstacles & - & 3 \\
\hline P2-P1 & 9 & Yes & -28 & 0 \\
\hline P2-P9 & 28 & Yes & -41 & 0 \\
\hline P1-P9 & 24 & Yes & -36 & 1 \\
\hline
\end{tabular}

for using antenna in our system. In Case- $B$, we move the node from position $P 1$ to $P 4$, which means that now it is attached to the concrete wall. As we previously mentioned that the pattern of our antenna is omnidirectional, this means that most of the signal is directly absorbed by the concrete walls around it. This explains the loss of signal strength from $-52 \mathrm{dBm}$ in Case- $A$ to $-62 \mathrm{dBm}$ in Case- $B$. Packet loss is also significantly increased.

\subsubsection{Test-6: Self-healing and reliability test.}

In this test, we placed our nodes at positions $P 1, P 3, P 4$ and $P 9$ as shown in Figure 3.10 and sent encrypted and secure XML files from $P 3$ to $P 9$. Results for this test are presented in Table 3.5. We used external antennas for signal strength and default PING parameters for this test. We observe from the signal strengths that nodes at position $P 1$ and $P 4$ and directly communicate with the node at $P 9$, but node at P3 cannot do the same because it has two buildings as obstacles which are strong enough to stop the signals. However, communication between $P 3$ and $P 9$ happens through $P 1$ or $P 4$ via mesh network. In order to simulate a node dropping scenario, we turn off $P 1$. We observed that the OLSR protocol automatically re-routed the communication through $P 4$ in the absence of node at $P 1$. This shows the self-healing characteristic of our system which means that even if one of the nodes goes down due 
to some reason, our communication system is not compromised and is fully reliable.

\subsubsection{Test-7: Interference effect}

Each channel on the $2.4 \mathrm{GHz} \mathrm{Wi}-\mathrm{Fi}$ spectrum is $20 \mathrm{MHz}$ wide. The channel centers are separated by $5 \mathrm{MHz}$, and the entire spectrum is only $100 \mathrm{MHz}$ wide. This means the 11 channels have to squeeze into the $100 \mathrm{MHz}$ available, and in the end, overlap. This causes interference. In our scenario, we are dealing with Co-Channel interference because all our nodes are on the same channel. In a co-channel interference environment, stations follow CSMA/CA and will check if the medium is busy. If the medium is idle, they will attempt to transmit data. If it is not idle, they will defer transmission until it is. Co-channel interference tnegatively impacts wireless networks when there is an increase in contention, for example a high-density area. As stations attempt to use the medium, they may need to wait for others to complete their transmissions. This explains the reason why we have different signal strengths between two nodes

placed at the same position ( $P 1$ and $P 3)$ in Test-5 and Test-6, as shown in Table 3.5 and 3.2. In Test-5, we observed $-50 \mathrm{dBm}$, however, it dropped down to $-52 \mathrm{dBm}$ in Test-6, because in Test-5, we only had two nodes activated, but in Test-6, we had four nodes communicating on the same channel in the same vicinity.

\subsubsection{Test-8: LTE connection between the TA and the BOS}

We connected the LTE shield with module and the antenna to the TA, and used Rogers network to connect to the BOS using LTE. Our BOS is set up at IBM cloud, so we configured the TAs and the NoSQL database connection using the IBM account credentials. A Node-Red application was set-up at both ends as shown in Fig 3.11 and secure connection based on TLS 1.2 was established. We confirmed the bi-directional 


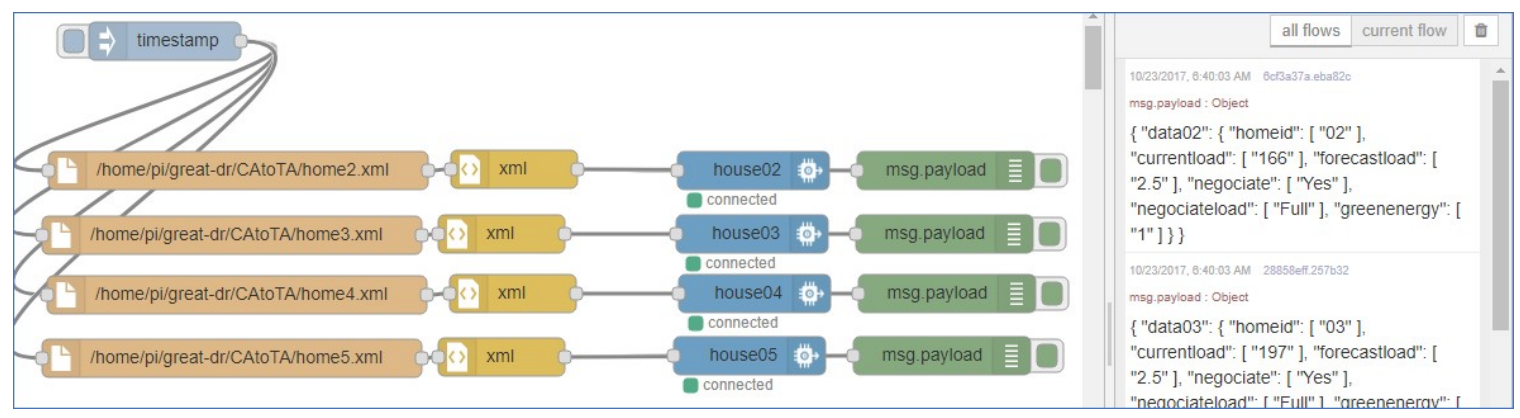

Figure 3.11: Node-Red application to connect TA and the cloud.

communication between the CAs and the cloud using the above mentioned set-up by sending the files generated by CAs to the cloud through the TA, storing them in the cloud database, and sending the modified version back to the CA through the TA. We further tested the stability of the LTE network by pinging google.com for more than 22 hours. Total 8023 packets were transmitted with 1\% packet loss and $160 \mathrm{~ms}$ as average round trip time.

\subsubsection{Sandcherry Site}

After extensive testing at Carleton University, we tested our system by deploying it in the real-homes because real-world scenarios are more complex and intense as compared to labs. Sandcherry site was carefully chosen by HOL for the implementation of TGDR system.

Layout: The top view of the site is shown in Fig 3.14. Layout from South to North is: House 24, House 22, House 20, House 18, maintenance room, laundry room, House 16, House 14, House 12, House 10. Four houses $\left(H_{10}, H_{16}, H_{18}\right.$ and $\left.H_{20}\right)$ have subscribed to the TGDR project so those were our testing and deployment targets. All these houses are located on the first floor. Distances between the key locations 


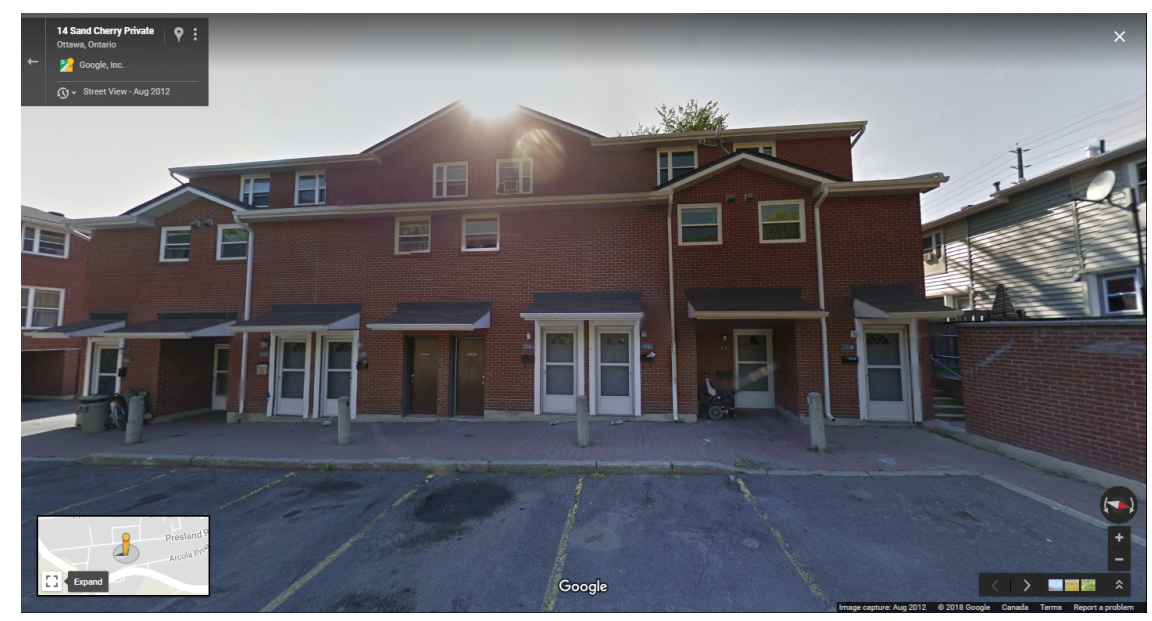

Figure 3.12: Front view of Sandcherry site.

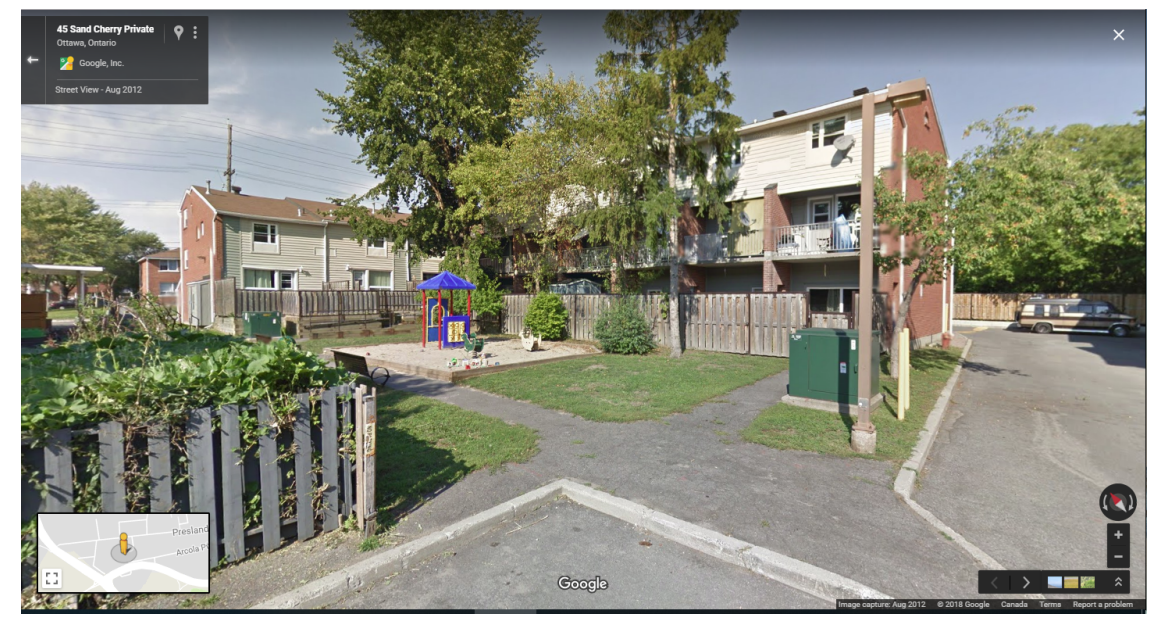

Figure 3.13: Back view of Sandcherry site.

are shown in Table 3.6.

Initial locations of the TA and CAs: All the TGDR-related equipment to any home (including $\mathrm{CA}$ ) was placed inside the storage room of that house, that is located at the front side of the home (towards the East end as shown in Fig 3.14). The TA is located in the transformer at the back of the houses towards the West end, whereas its antenna is mounted outside the transformer and connected to the TA using a low 


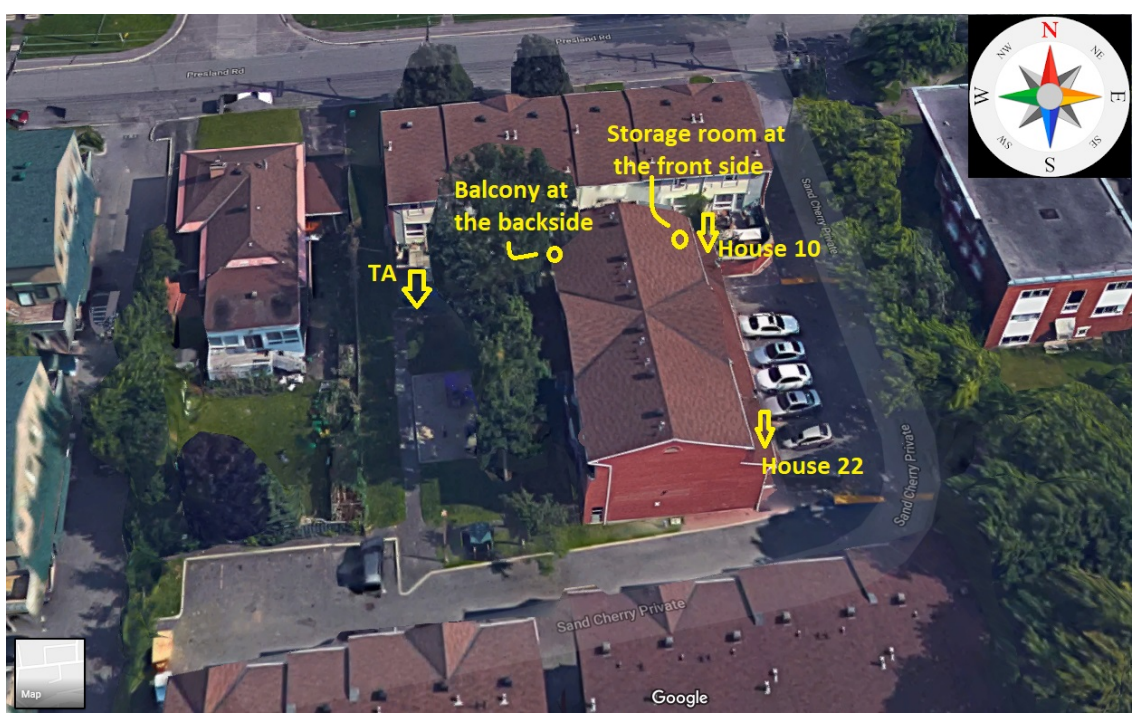

Figure 3.14: Top view of Sandcherry site

loss cable.

Table 3.6: Distance table for Sandcherry site (meters).

\begin{tabular}{|l|c|c|c|c|c|c|c|c|}
\hline From $\backslash$ To & $H_{10}$ balcony & $H_{10}$ storage & $H_{16}$ balcony & $H_{16}$ storage & $H_{18}$ balcony & $H_{18}$ storage & $H_{20}$ balcony & $H_{20}$ storage \\
\hline TA & 13.2 & 25.5 & 17.1 & 28.7 & 19.5 & 30.9 & 23.3 & 32.8 \\
\hline$H_{10}$ balcony & 0 & 13.2 & 8 & 15 & 12.6 & 17.3 & 16.4 & 22 \\
\hline$H_{10}$ storage & & 0 & 15 & 8 & 17.3 & 12.6 & 22 & 16.4 \\
\hline$H_{16}$ balcony & & & 0 & 13.2 & 4.6 & 14.2 & 8.5 & 16 \\
\hline$H_{16}$ storage & & & & 0 & 14.2 & 4.6 & 16 & 8.5 \\
\hline$H_{18}$ balcony & & & & & 0 & 13.2 & 4 & 14.2 \\
\hline$H_{18}$ storage & & & & & & & & \\
\hline$H_{20}$ balcony
\end{tabular}

The details of our tests and propagation survey are explained in the next subsections. The parameters for these tests are described in Table 3.7. We selected WiFi 
Table 3.7: Parameters for Sandcherry site testing.

\begin{tabular}{|c|c|}
\hline Parameter & Value \\
\hline Packet size & 5000 bytes payload +8 bytes header \\
\hline Packet sending interval & $0.5 \mathrm{sec}$ \\
\hline Number of Packets & 100 \\
\hline Frequency & $2.412 \mathrm{GHz}($ Channel 1$)$ \\
\hline
\end{tabular}

channel-1 after analyzing the network traffic on all the channels through network analyzer tool. We found around 35 SSIDs working in that area, and channel-1 had the least number of users.

\subsubsection{Test-1: Without external antenna}

Initially, we tested the communication between TA located beside the transformer and $\mathrm{CA}$ located in the storage room of $H_{10}$. The communication was very weak because of very low signal strength. The reason was two concrete walls (external wall of the balcony and internal wall of the storage room). Moreover, the TA was at ground level and the homes are on the first floor, which means there is a significant signal drop due to concrete flooring as well. We then moved the CA to the balcony from the storage room and observed a significant improvement in the overall network performance as shown in Table 3.8 .

Conclusions: Based on this test, we concluded that either we need to use external antenna for more power, or we need to add another node at the balcony to act as a relay between $\mathrm{TA}$ and $\mathrm{CA}$ in the storage room. We explore the performance of external antenna in the next test. 
Table 3.8: Communication from TA to $H_{10}$ without external antenna

\begin{tabular}{|c|c|c|c|}
\hline CA location & Signal Strength $\mathbf{( d B m )}$ & Packet Loss (\%) & Latency (ms) \\
\hline Storage Room & -79 & 76 & 7588 \\
\hline Balcony & -69 & 28 & 220 \\
\hline
\end{tabular}

\subsubsection{Test-2: With external antenna}

Based on Test-1 results, we decided to use external antenna as they were proven to improve the performance significantly during the tests at Carleton University (refer to Test-4 in Section 3.3.1.5). This was one of our most extensive test in which we placed a relay at the balcony of $H_{10}$ and CAs in the storage rooms of $H_{10}, H_{16}, H_{18}$ and $H_{20}$ to perform end to end testing by using various packet sizes. The signal strengths we observed are described in Table 3.9 and packet loss and latency results are described in Table 3.10. We draw the following observations from these tables:

- There is a significant improvement in the signals as compared to Test-1 in which we were not using external antenna.

- The communication between TA and relay is very stable but if the relay is removed, then we still have a weak signal strength $(-67 \mathrm{dBm})$ between TA and $H_{10}$ storage room despite using external antennas.

- In this scenario, all generated traffic by the CAs need to go through the relay in order to reach the TA. Therefore, this transforms the Mesh architecture into a single-path multi-hop relaying.

Conclusions: Based on the above observations, we conclude that we must need a relay at the balcony to ensure the communication between all the houses, else we need to move the TA closer to the storage room. Next test will explain further details. 
Table 3.9: Signal strength (in $\mathrm{dBm}$ ) between the nodes for Test- 2 .

\begin{tabular}{|c|c|c|c|c|c|}
\hline From/To & Relay & $H_{10}$ & $H_{16}$ & $H_{18}$ & $H_{20}$ \\
\hline TA & -53 & -69 & -74 & -75 & -78 \\
\hline Relay & & -40 & -66 & -69 & -71 \\
\hline$H_{10}$ & & & -52 & -55 & -62 \\
\hline$H_{16}$ & & & & -49 & -57 \\
\hline$H_{18}$ & & & & & -54 \\
\hline
\end{tabular}

Table 3.10: Network performance from TA to various destinations for Test-2.

\begin{tabular}{|c|c|c|}
\hline Destination & Packets Lost (\%) & Latency (ms) \\
\hline Relay & 0 & 23 \\
\hline$H_{10}$ & 2 & 65 \\
\hline$H_{16}$ & 0 & 66 \\
\hline$H_{18}$ & 4 & 67 \\
\hline$H_{20}$ & 6 & 91 \\
\hline
\end{tabular}

\subsubsection{Test-3: Solutions for relay}

From the previous test, we established the need for adding a relay at the balcony of $H_{10}$ to ensure smooth communications which required powering arrangements.

Powering the relay: We have different options to power the relay:

- Power it using the household power supply from the balcony. This was not possible as it was against the contract with the residents to use multiple power outlets. The only permission we had was to use the storage room power supply.

- Run a power cable from the storage room to the balcony. This was considered as an expensive option as it involved the cost of hardware for relay along with the cost of power cable and the circuit. 
Considering the installation of the relay as a non-viable option, we proposed to change the position of the TA, which means to move it towards the East to make it closer to the balcony, or change its height.

Moving the TA: Several tests were performed to determine the viability of moving the TA. Initially we increased the height of the antenna of the TA above the transformer using a pole. Although there was a slight improvement in the signal strength, but it didn't help much. This may be due in part to the amount of metal on the west balcony. There is a metal fence and railing, and a bicycle, and a large metal BBQ, all up to half the open height of the balcony. Even on a pole, signal strength between the TA and $\mathrm{CA}$ in storage room was around $-67 \mathrm{dBm}$ and packet losses about $15 \%$ which was not sufficient for good links.

Further we probed just outside the $H_{10}$ patio, with a pole up to about middle height of the balcony. We observed the signal strength improved significantly to -55 $\mathrm{dBm}$ and packet loss was around $1 \%$. This location is about $6 \mathrm{~m}$ closer than the transformer, and eliminates about half the tree greenery. This seems to be a good signal strength but it needs trenching from the transformer pedestal over to the patio fence, to extend the RF and power cables. However, trenching is not so cheap so this option was avoided. Moreover, the RF cable (RG-58) had a loss of $4.2 \mathrm{~dB}$ for 16.4 feet which is a considerable amount. Results for this test are summarized in table 3.11 and in Fig 3.15 .

Conclusions: Running the power cable east to west of the house to power the relay was not a cheap option, so was the trenching to move the TA. Therefore, we decided to consider other options as explained in the next section. 
Table 3.11: Results for Test-3 at Sandcherry (refer to Fig 3.15 for TA positions).

\begin{tabular}{|c|c|c|}
\hline TA position & Signal Strength $(\mathbf{d B m})$ & Packet Loss (\%) \\
\hline A and B & -65 to -70 & 15 \\
\hline C & -63 to -67 & 8 \\
\hline D & -57 to -61 & 6 \\
\hline E & -55 & 1 \\
\hline
\end{tabular}

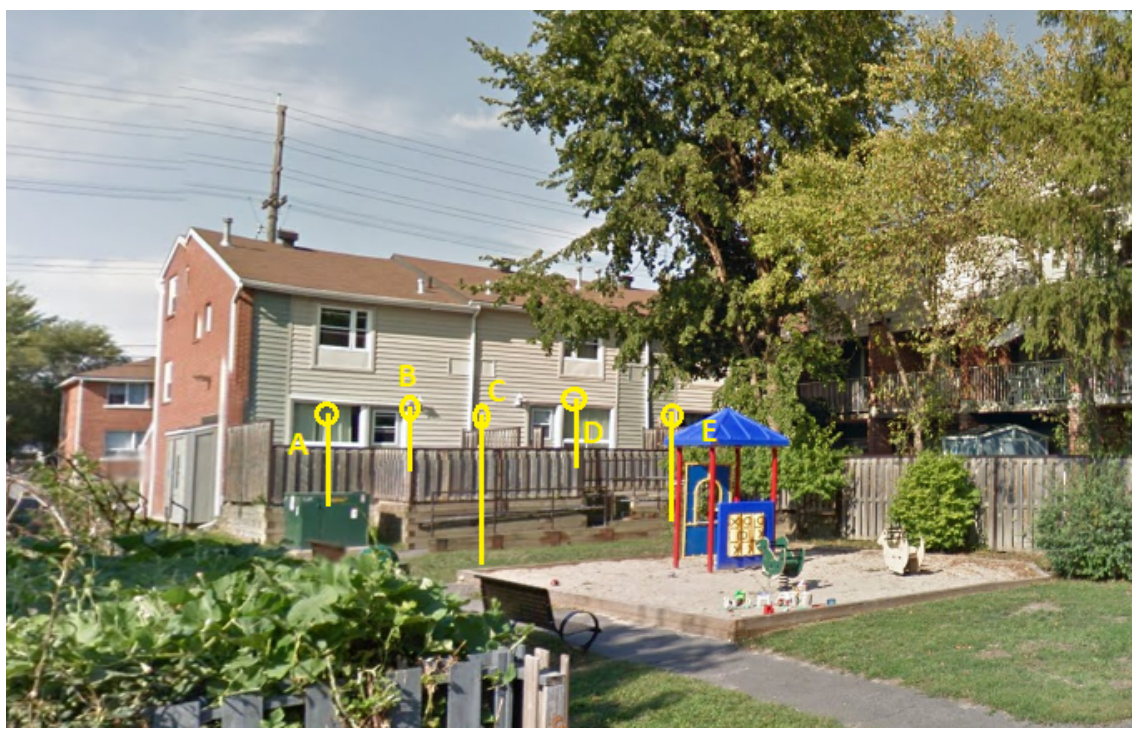

Figure 3.15: Various TA positions for Test-3 at Sandcherry site.

\subsubsection{Test-4: Low Loss RF Cable solution}

After disregarding the above options to solve the relay problem, we decided to test an RF cable running from the East to West end of each home (from the storage room to the balcony). This means that our CA remains in the storage room, connected with HEMSC, however, the antenna for the CA is placed at the balcony and is connected to the $\mathrm{CA}$ via $\mathrm{RF}$ cable. In this test, we studied the signal strength between different nodes with and without the placement of neighbouring nodes to note the effect of interference. Co-channel interference is a well-known phenomenon in wireless networks and has proved to degrade the communication links as proven in 
CHAPTER 3. IMPLEMENTATION OF THE SMART GRID COMM. SYS.

Table 3.12: Signal strength (in $\mathrm{dBm}$ ) from TA to various destinations for Test-4.

\begin{tabular}{|c|c|c|c|}
\hline \multirow{2}{*}{ Destination } & \multicolumn{3}{|c|}{ Signal Strength $(\mathrm{dBm})$} \\
\cline { 2 - 4 } & Neighbours: None & Neighbours: $H_{10}, H_{18}$ & Neighbours: $H_{10}, H_{16}, H_{18}$ \\
\hline$H_{10}$ & -53 & -57 & -60 \\
\hline$H_{18}$ & & -55 & -58 \\
\hline$H_{16}$ & & & -59 \\
\hline
\end{tabular}

Table 3.13: Network performance from TA to various destinations for Test-4.

\begin{tabular}{|c|c|c|c|}
\hline Destination & Packet Size (kbytes) & Packets Lost (\%) & Latency (ms) \\
\hline$H_{10}$ & {$[5,4,3,2,1]$} & {$[0,0,0,0,0]$} & {$[35,33,45,13,8]$} \\
\hline$H_{16}$ & {$[5,4,3,2,1]$} & {$[7,7,13,3,4]$} & {$[172,138,107,158,42]$} \\
\hline$H_{18}$ & {$[5,4,3,2,1]$} & {$[6,4,4,5,3]$} & {$[58,56,48,36,28]$} \\
\hline
\end{tabular}

Section 3.3.1.7. Moreover, the RF cable has its own loss which was initially calculated to be $1.1 \mathrm{~dB}$ per meter because we were using a normal RF cable (RG-58). This was a significant loss, therefore, we decided to move with Low loss Siretta LLC200A RF cable, which has a loss of only $0.55 \mathrm{~dB}$ per meter [85].

In Table 3.12, we present the signal strengths' results. Furthermore, we study the packet loss and the latency for various packet sizes. These results are described in table 3.13 .

Conclusions: The final testing of running Low Loss RF cable from East to West proved to provide the best communication links at the cheapest cost, offering a reliable mesh network so that each node is connected through multiple routes to the TA. We also concluded that changing the height of TA along the pole by approx one foot significantly changes the signal strength (it was increased from $-62 \mathrm{dBm}$ from -57 $\mathrm{dBm})$. This can be attributed to the reflections from metal railings nearby or the metal objects placed in the balcony of the House-10. Therefore, we propose a sliding 
pole to adjust the height of the TA to get the best signals during final installation of TGDR equipment.

\subsubsection{Viewmount Site}

Viewmount is the second site where we deployed our communication system.

Layout: The East and North-West view of the Viewmount site is shown in Fig. 3.16(a) and 3.16(b) respectively. Layout from South to North for the ground floor on the East side is: House 102, House 104, House 106, House 108, House 110. The West side has odd number of houses. Same layout is replicated for the first floor. Five houses $\left(H_{107}, H_{207}, H_{206}, H_{208}\right.$ and $\left.H_{210}\right)$ have subscribed to the TGDR project from this site. They are distributed on ground floor and first floor as indicated by the first digit of the house number. The location of CAs was pre-determined based on the placements of the thermostats in the houses, which made this site relatively less complex than Sandcherry. We also placed a CA in the electrical room for PV panels.

Results: We use our propagation survey results from Carleton University and Sandcherry site here at Viewmount as well, so all the tests are not described again, for example, use of external antenna etc. This site is still under deployment but so far the tests have been very successful. Results of the signal strengths are shown in Table 3.14, and packet loss and latency are described in Table 3.15.

Conclusions: Based on the results, we observe that the communication is working well at Viewmount and satisfies all the requirements of TGDR, however, we propose to add a relay between the node in the electrical room and the node in $H_{107}$. This 


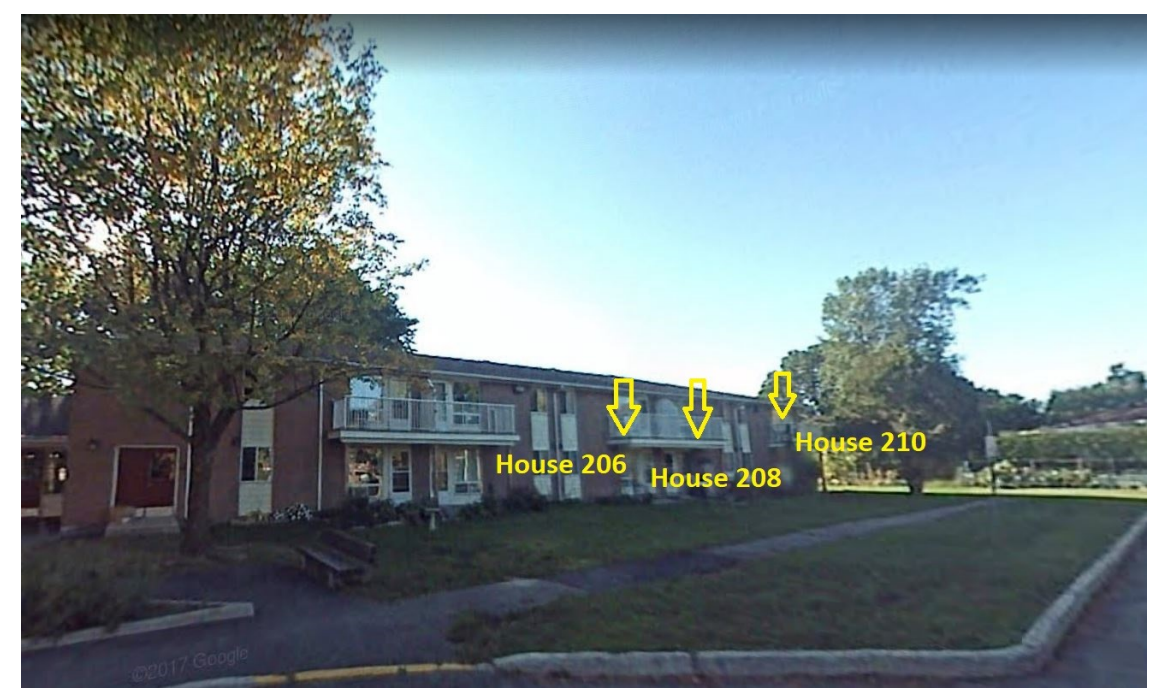

(a) Eastern side of the Viewmount site.

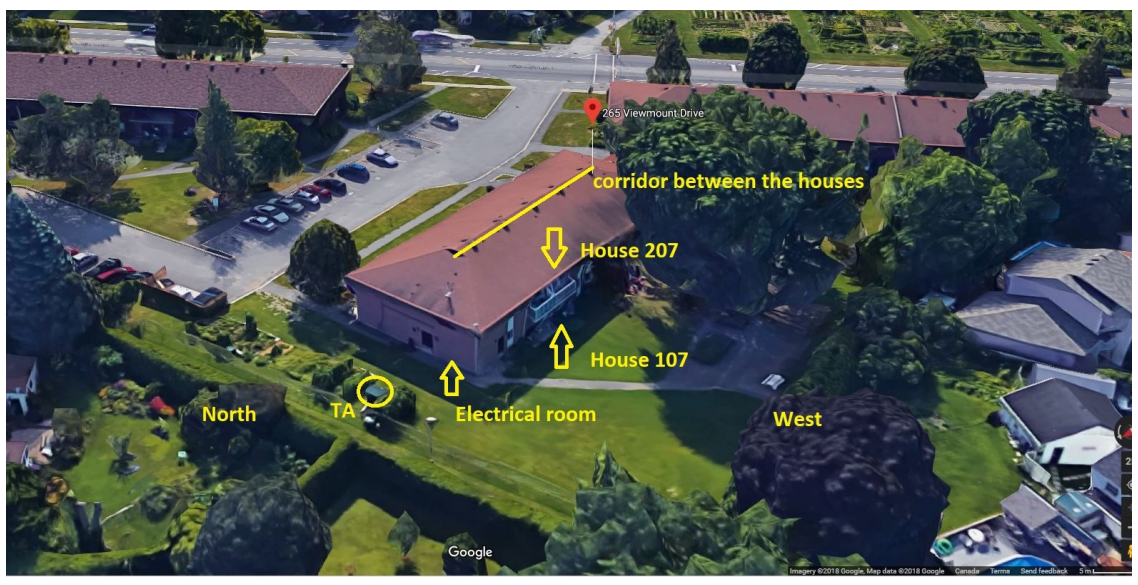

(b) North and West view of Viewmount site.

Figure 3.16: Layout of Viewmount site.

Table 3.14: Signal strength (in dBm) for Viewmount site.

\begin{tabular}{|c|c|c|c|c|c|}
\hline From/To & Electrical room & $H_{210}$ & $H_{107}$ & $H_{208}$ & $H_{207}$ \\
\hline TA & -45 & -61 & -65 & -71 & -73 \\
\hline Electrical room & & -59 & -64 & -70 & -72 \\
\hline$H_{210}$ & & & -62 & -55 & -61 \\
\hline$H_{107}$ & & & & -63 & -59 \\
\hline$H_{208}$ & & & & & -58 \\
\hline
\end{tabular}


Table 3.15: Network Performance from TA to various destinations: Viewmount site.

\begin{tabular}{|c|c|c|c|}
\hline Destination & Packet Size (kbytes) & Packets Lost (\%) & Latency (ms) \\
\hline Electrical room & {$[5,4,3,2,1]$} & {$[0,0,0,0,0]$} & {$[14,17,12,7,91]$} \\
\hline$H_{107}$ & {$[5,4,3,2,1]$} & {$[1,0,0,0,0]$} & {$[25,28,17,12,6]$} \\
\hline$H_{210}$ & {$[5,4,3,2,1]$} & {$[4,0,4,6,0]$} & {$[100,76,59,64,22]$} \\
\hline$H_{208}$ & {$[5,4,3,2,1]$} & {$[2,4,8,0,0]$} & {$[204,131,95,49,36]$} \\
\hline$H_{207}$ & {$[5,4,3,2,1]$} & {$[12,9,10,10,8]$} & {$[990,657,322,101,73]$} \\
\hline
\end{tabular}

will further enhance the stability and reliability of the system.

\subsection{Summary}

In this chapter, we explain the deployment of our communication mechanism based on IEEE 2030.5 in real-world environment and present our testing results. We proved that our communication system is reliable and satisfies all the requirements mentioned in Section 3.2.3. Moreover, it is scalable and multiple CAs can be added as needed in the mesh network, with obvious constrains of congestion and interference. After the successful communication of energy data between CAs, the TA and the cloud, it needs to be processed and analyzed to get the most use of it. Therefore, in the next chapter, we explain our work on data analytics and forecasting. 


\section{Chapter 4}

\section{Smart Grid Data Analytics}

\subsection{Introduction}

In this chapter, we evaluate and discuss energy forecasting using different models for short and long-term predictions. Then, we present our proposed approach based on ensemble models. We show that our proposed approach has better performance than existing models. This chapter is organized into two main sections. Section 4.2 starts with explaining the system model and our methodology, followed by data sources that are used in this thesis. Next, we analyze data and show important visualizations. We then explain the pre-processing steps required before applying the forecasting models. Finally, we explain two different prediction scenarios to be used for energy forecasting. Section 4.3 presents the performance and evaluation of our forecasting models. It starts with results for scenario-1, where we evaluate and discuss the predictions obtained using various models. Next, we present the results for scenario-2 which also includes the results obtained using our proposed approach based on ensemble modelling. Section 4.4 concludes this chapter. 


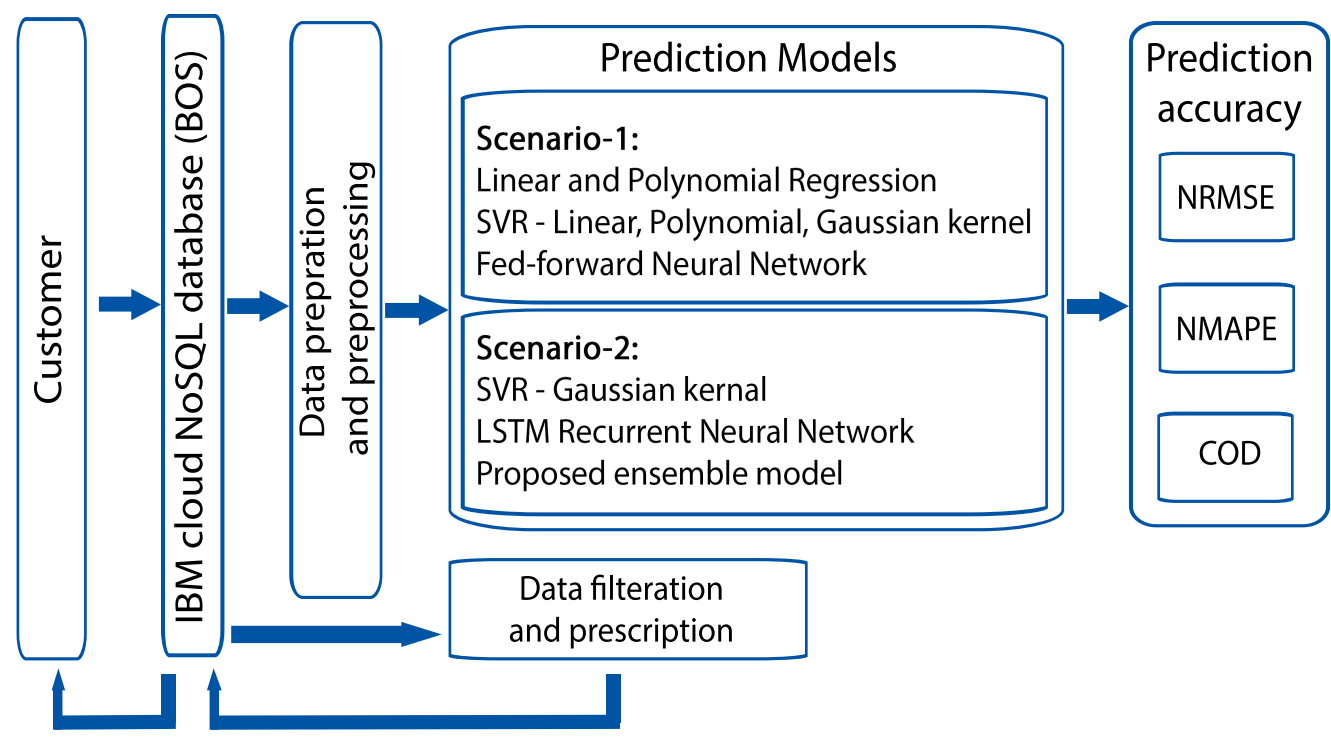

Figure 4.1: System model for data processing and analytics.

\subsection{Prediction Engine}

In order to analyze the electricity data and test our forecasting models explained in Section 2.3.2, we use the dataset from MIT as detailed in Section 4.2.1 because the data from our communication system is not yet mature. Different parts of the analytics are shown in the system model diagram in Fig. 4.1 which mainly include visualization, recommendations, preprocessing, modelling and forecasting. All these modules are explained in details in the following subsections.

\subsubsection{Dataset}

The dataset used is obtained from UMASS laboratory of MIT [86]. We extract a collection of energy consumption values (measured every 15 minutes in kilowatthour (kWh) of 25 single resident apartments collected from January 1 to December 31, 2015. This dataset also provides us with weather data including temperature, 


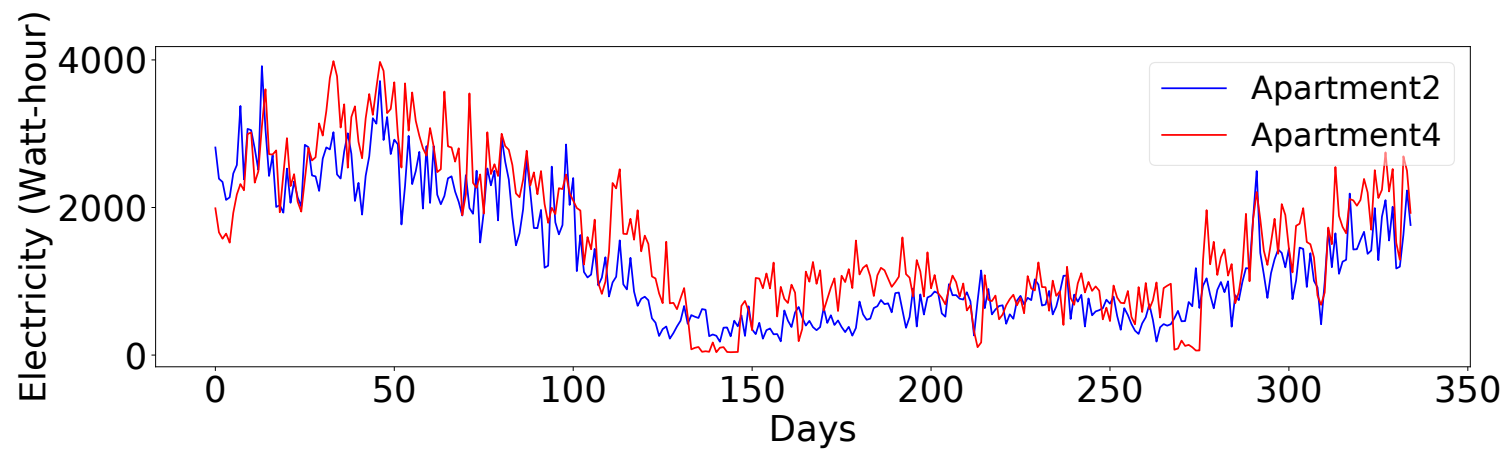

(a) Time's effect on consumption.

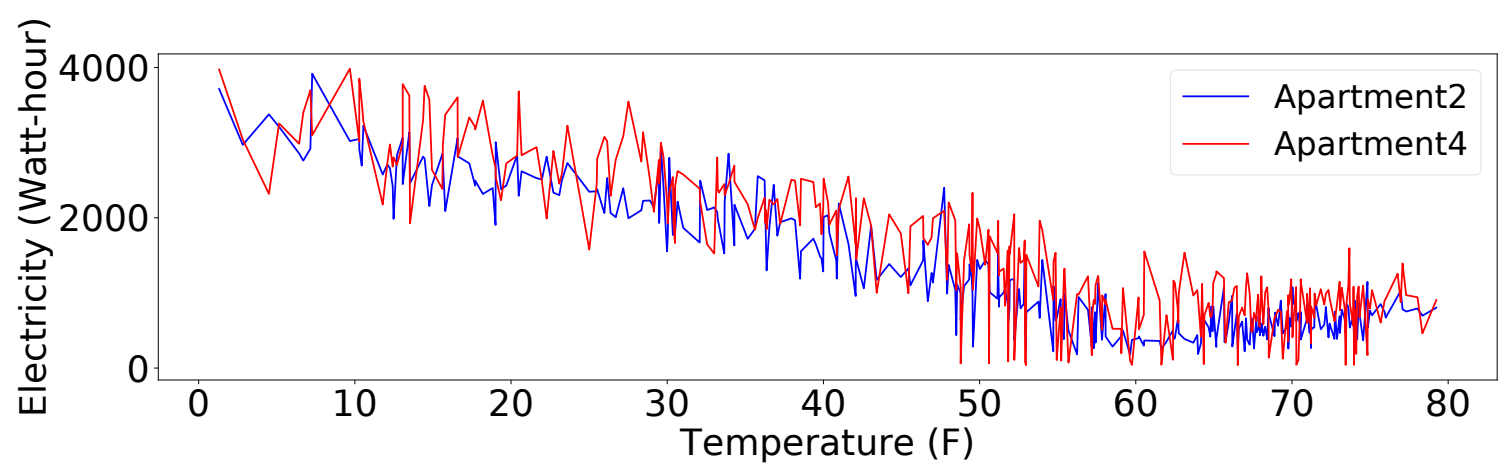

(b) Temperature's effect on consumption.

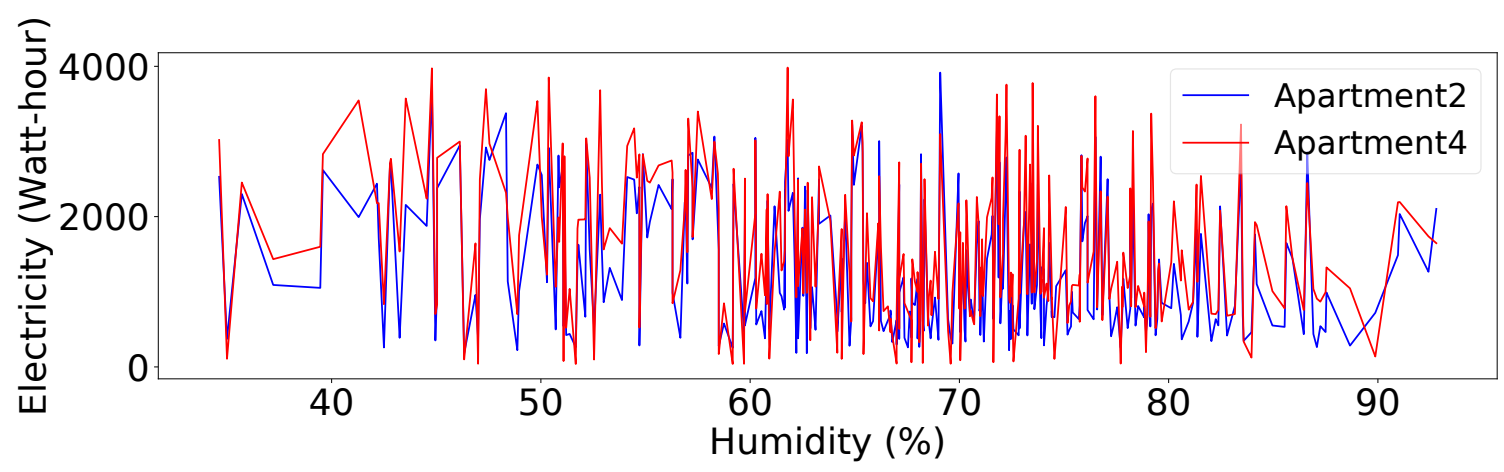

(c) Humidity's effect on consumption.

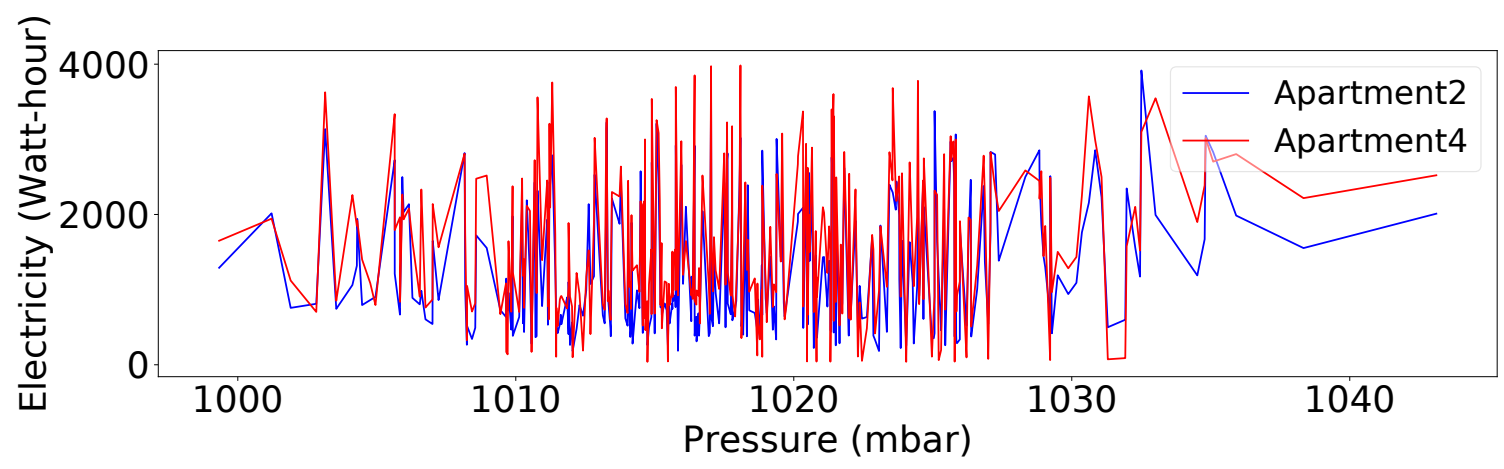

(d) Pressure's effect on consumption.

Figure 4.2: Energy consumption as a function of different parameters. 
pressure, humidity, and wind speed for the year 2015 where data has been sampled every hour. We store and process this data in IBM cloud services.

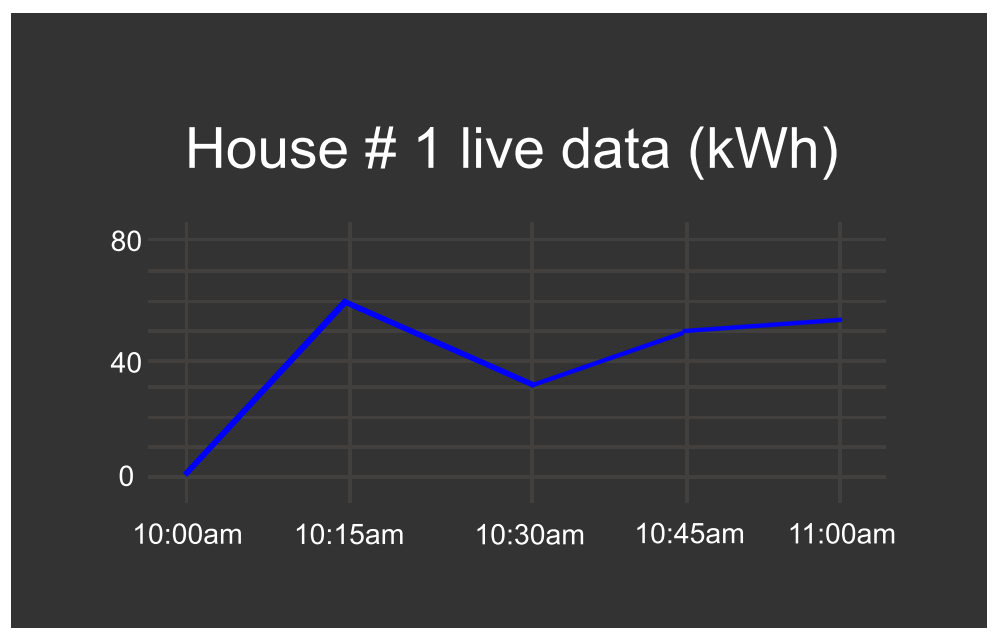

Figure 4.3: Real-time power consumption.

\subsubsection{Data Visualization}

The stored data in the cloud is graphically visualized and analyzed by the utility company. Fig. 4.2 shows how the energy consumption for two houses is being affected by change in time, temperature and other factors. Fig. 4.3 shows the real-time changing electric consumption of a particular house (the consumption of house- 1 in $\mathrm{kWh}$ is presented here). It refreshes every 15 minutes.

Fig. 4.4 shows the collective display of four homes in a neighbourhood . Fig. 4.5 tells the utility which house is consuming the most and the least energy. Moreover, a warning will be raised if any house has not opted for negotiation with the utility, and still exceeds the recommended limit of energy consumption. Negotiation parameter is one of the attributes that customers can set as explained in Section 3.2.3.3, which 

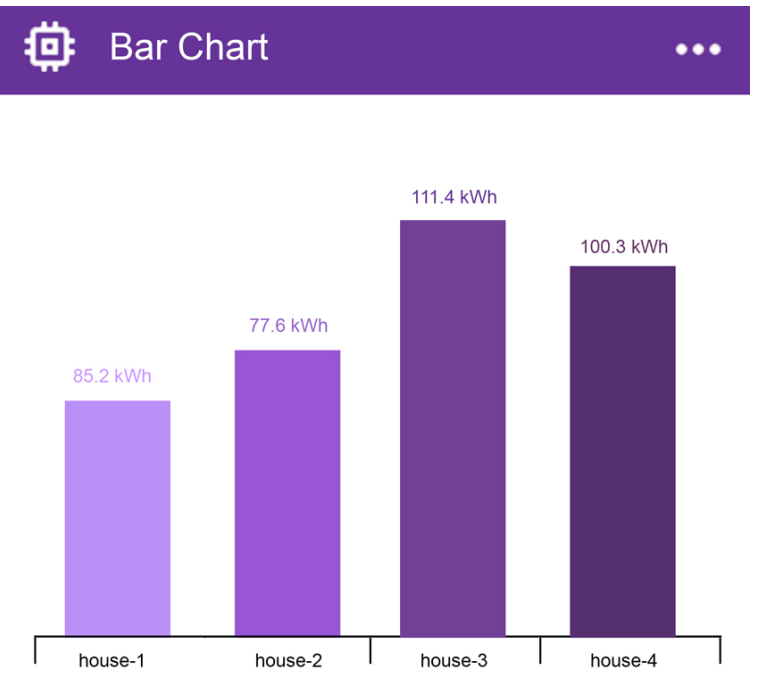

Figure 4.4: Comparison of current power consumption of four neighbouring houses.

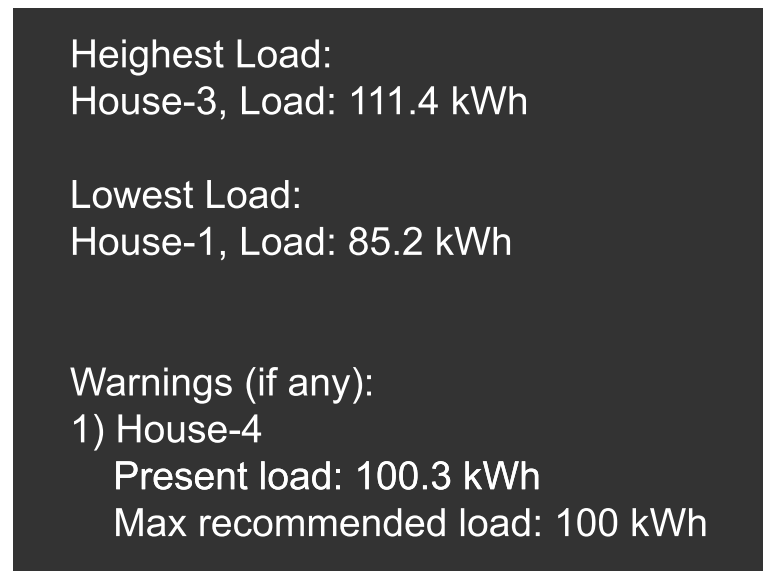

Figure 4.5: Highest and lowest loads in the neighbourhood, along with warnings from the utility to the houses.

shows whether or not they have opted to negotiate. In this case, the recommended energy consumption limit set by the utility is $100 \mathrm{kWh}$, but house- 3 and house- 4 are exceeding the limit. House-3, however, has opted for negotiation so utility is able to negotiate with that anytime, but house- 4 has opted out of negotiation so the utility will alert it with a warning sign. This negotiation process can be elaborated more through the implementation of demand response algorithms offering real-time pricing 
to provide an advantage to users opting in. All of this will serve to reduce the overall load on the grid while serving customers' benefits.

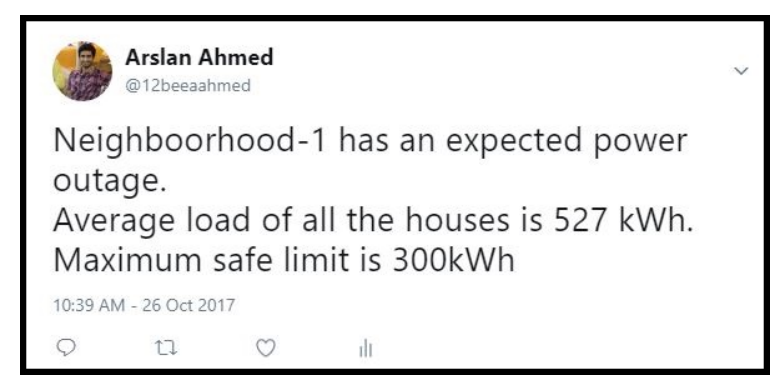

Figure 4.6: Tweet by the utility if outage is predicted in a given neighbourhood.

Furthermore, we have shown in Fig. 4.6 that the utility can timely warn the consumers via tweets if a neighbourhood is collectively consuming more energy than the recommended limit, and is expected to exceed the amount that the grid can handle, resulting in a power outage. This will enable the users to make any necessary preparations beforehand. As a further display of the data, we present the energy consumption as line or bar graphs showing the daily, weekly, and monthly trends in power consumption for a single house or an entire neighbourhood as shown in Fig. 4.7. Such visual analytics provide an insight into the power consumptions patterns

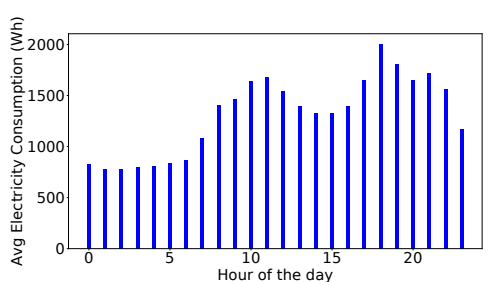

(a) Daily

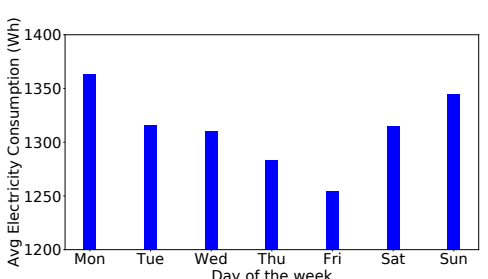

(b) Weekly

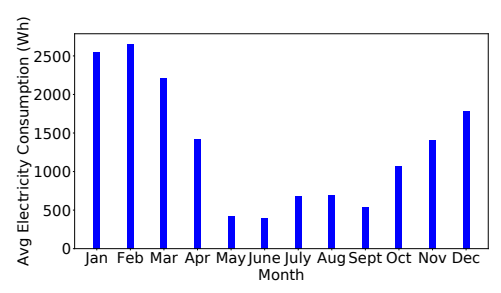

(c) Monthly

Figure 4.7: Power consumption displayed for different time scales.

including peak hours, days, and seasons. Based on the hourly analysis of the data, we observed that users tend to consume more energy at night as compared to the day. 
The reason behind this can be attributed to the living style of the residents, who tend to spend most of their daytime at work. We have also filtered the apartments based on high and low consumption, considering their monthly average. This can help to classify rich and poor residents and change the electricity rates accordingly.

\subsubsection{Data Preparation and Pre-processing}

Data pre-processing is an important step in the before applying any prediction model. In fact, it takes about $80 \%$ of the efforts to pre-process the data and make it ready for the model, rest of $20 \%$ effort is to choose the correct model and apply it properly. The phrase "garbage in, garbage out" is particularly applicable to data mining and machine learning projects [87]. Therefore, analyzing data that has not been carefully screened for such problems can produce misleading results.

The high importance of data preprocessing can be attributed to the following reasons: Data-gathering methods are often loosely controlled, resulting in out-ofrange values (for example negative income), impossible data combinations, noise or missing values, or sometimes inconsistent values. Most of the times we have data from different sources and we need to aggregate them together based on common values before applying our models.

The dataset obtained at the cloud from CAs was not fit for applying the models directly, so we pre-processed the data by performing various operations on the data as detailed below.

Re-sampling: Generally, we have to resample our data if there are multiple sources and they do not have the same resolution in case of time series data. We may upsample or down-sample our data. In case of down-sampling, popular methods include taking the mean or the sum. In case of up-sampling, popular methods include linear 
interpolation, forward fill (take the next known value), and backward fill (take the last known value).

In our dataset, energy readings are recorded based on 15 min intervals and weather readings are recorded based on hourly intervals, so we first up-sample the weather readings and down-sample to energy readings to half-hourly resolution because we need same resolution of variables. While resampling, we use the most commonly used linear interpolation technique to determine the unknown temperature value, and mean value for down-sampling the energy data.

Data aggregation: This step is performed to integrate data from different sources into one file. This makes it easier to process the data as a whole, for example finding correlations between to columns etc. Since our dataset was obtained from two files (energy and weather), we aggregate them together based on time as an index.

Outliers removal: An outlier is an observation that appears to deviate significantly from other observations in the sample. It may indicate bad or noisy data, for example, the sensor may not be running correctly at that point of time [88]. Such data should be dealt appropriately otherwise it can skew the results. We detected many outliers in our dataset, for example, very high values of energy consumption. This was done by plotting a histogram of the dependent variables. Fig 4.8(a) shows the histogram of energy consumption data. we apply univariate method which looks for data points with extreme values on each variable, and drop the rows that have outliers. To select which rows to drop, we calculated $99^{\text {th }}$ percentile of the energy data and removed all points that lie above it. Using this method, we made sure that our dataset still represents $99 \%$ of the original dataset but the histogram has significantly changed as shown in Fig 4.8(b). In some critical scenarios, these outliers may have a strong 


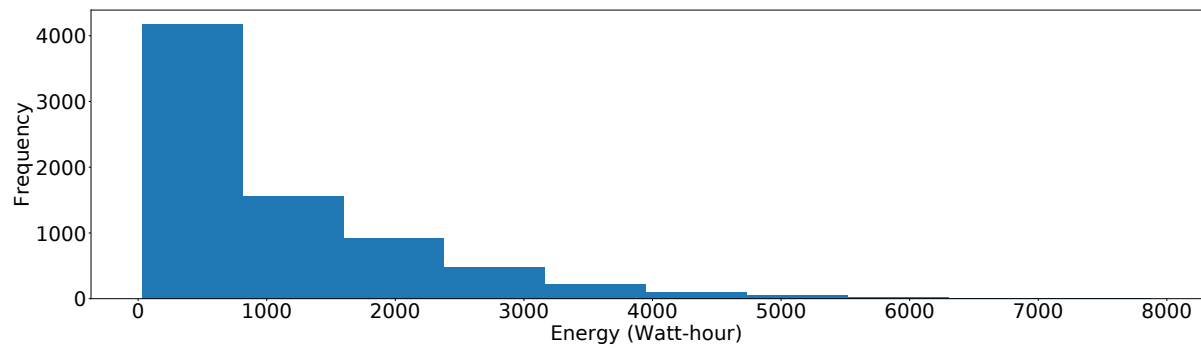

(a) Before removing outliers.

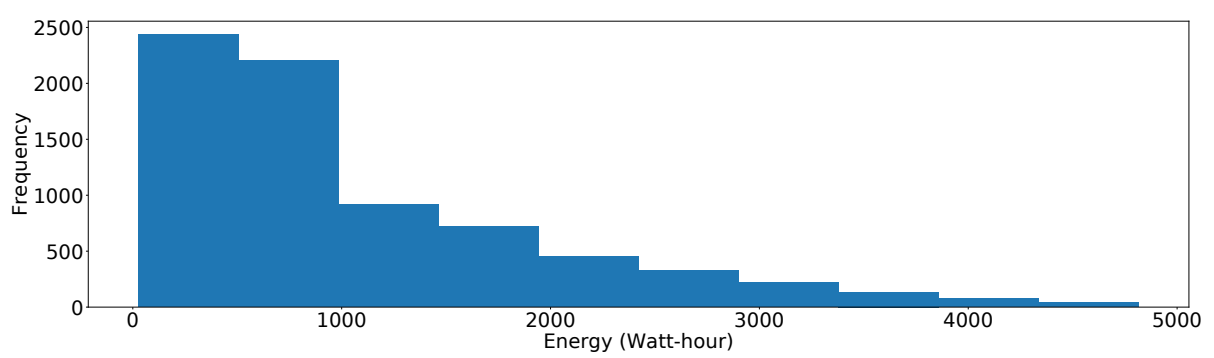

(b) After removing outliers.

Figure 4.8: Histogram of energy data before and removing outliers.

significance and should not be removed, however, those cases are out of our scope.

Handling missing values: For various reasons, a dataset can have some missing values, often encoded as blanks, not a number, or other placeholders. Such datasets are incompatible with many scikit-learn [89] estimators which assume that all values in an array are numerical, and that all have meaning. Our dataset also had some missing values, partly because of the sensor not working at that time, or the communication system got disrupted momentarily, or because of removing outliers. A simple strategy to use incomplete datasets was to discard entire rows and columns containing missing values. However, this comes at the price of losing valuable information. Therefore, we opted for a better strategy of imputing the missing values by inferring them from the known part of the data using linear interpolation method. 
Table 4.1: List of features.

\begin{tabular}{|c|c|}
\hline Feature & Nature \\
\hline Temperature & Continuous \\
Humidity & Continuous \\
Visibility & Continuous \\
Apparent Temperature & Continuous \\
Dew Point & Continuous \\
Pressure & Continuous \\
Wind Bearing & Continuous \\
\hline Precipitation Intensity & Continuous \\
Icon & Categorical \\
Cloud Cover & Categorical \\
Summary & Categorical \\
\hline
\end{tabular}

Remove correlated features: In statistics, multi-collinearity is a phenomenon in which one predictor variable in a multiple regression model can be linearly predicted from the others with a substantial degree of accuracy. In this situation, the coefficient estimates of the multiple regression may change erratically in response to small changes in the model or the data [90]. This is because most of the models tend to over-weigh them. For example, if you have length in inches and feet, they will be fully correlated and the model may give twice the weight to the length variable than it should have. Moreover, large number of features also make the model slower. Due to the curse of dimensionality, less features usually mean high improvement in terms of speed. Therefore, we first identify and then remove the correlated features from our dataset. Our original set of features is given in Table 4.1. We use Pearson correlation coefficient [91] to compute the correlation between numeric features and draw a heat map shown in Fig 4.9. As evident from heat-map, only the temperature feature has good correlation with our dependent variable, which is energy consumption in 
our case. Apparent temperature is also correlated with energy consumption but it is highly correlated with actual temperature so we decided to remove it. Similarly, other features including dew point are removed.

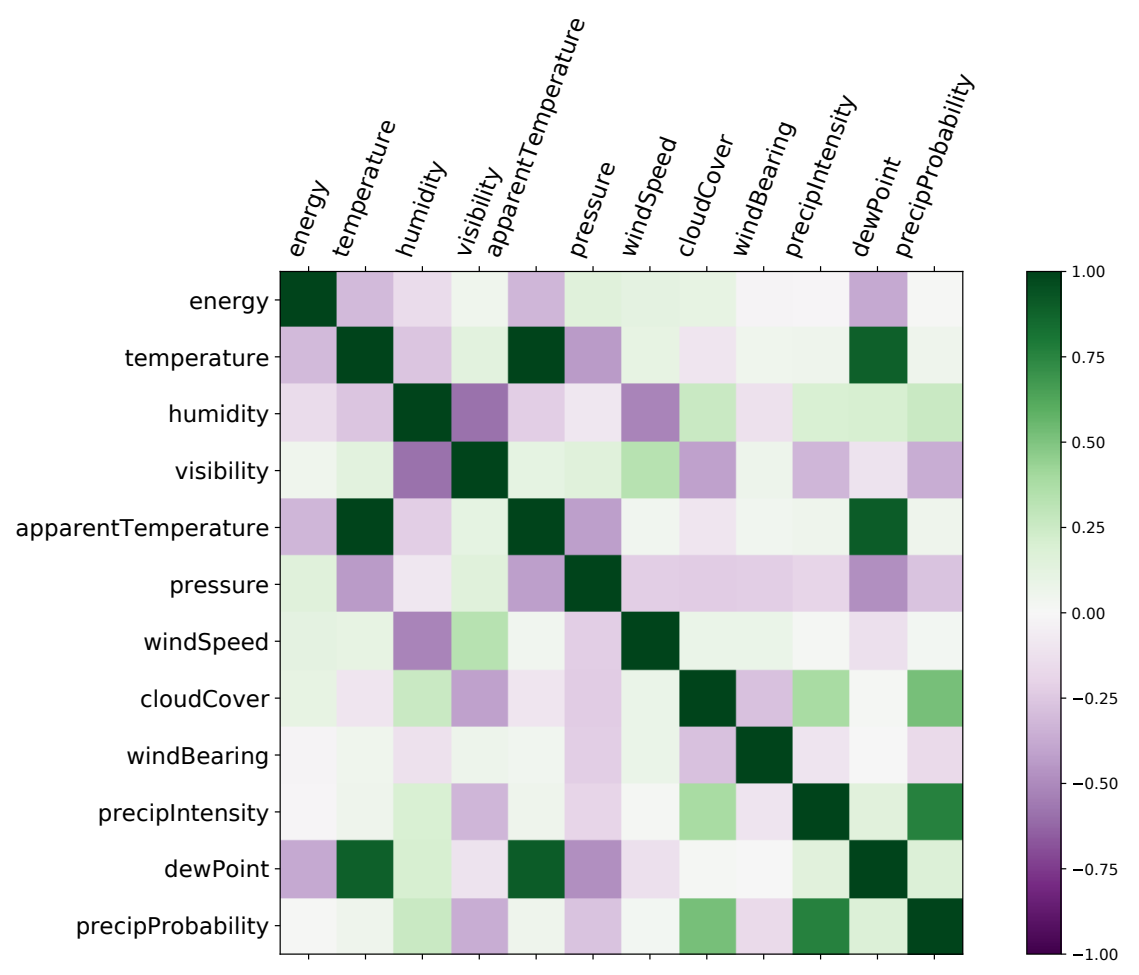

Figure 4.9: Heat-map of feature correlation.

Feature extracting: Extracting features from the given data is one of the core steps in data processing, specially when we are dealing with time series forecasting. In this case, we cannot use time as a direct feature because each time stamp is unique, and if we directly input it as a feature, our model will never see any time stamp again. This is against the concept of machine learning because our model is not learning anything from timestamp as none of it repeats itself. It is just the sequence and regularity that matters. Therefore, in order to process timestamps as a feature, we extract further features from it that repeat after regular intervals. This 
Table 4.2: One hot encoding of categorical features.

\begin{tabular}{|c|c|c|}
\hline Icon & Cloud Cover & Summary \\
\hline 1 & 0 & 0 \\
\hline 0 & 1 & 0 \\
\hline 0 & 0 & 1 \\
\hline
\end{tabular}

way, our model is able to learn patterns. For example, in our case we have extracted hour of the day, day of the week, date of the month, month of the year, and whether or not its a holiday. Moreover, we also need to re-frame our data to be treated as a supervised learning problem. This means that we create lag variables of a constant window size called history. We take the history of size $n(t, t-1, t-2, \ldots, t-n)$ as the feature vector and the next value $(t+1)$ as the dependent variable.

One hot encoding: Categorical data are variables that contain label values rather than numeric values, for example: A "colour" variable with the values: "red", "green" and "blue". Many machine learning algorithms cannot operate on label data directly. They require all input variables and output variables to be numeric. Sometimes, we may replace them with 1, 2 and 3 if they are ordinals (this is called Integer encoding). In our data, features such as weather icon and day of the week etc. are the categorical features where each value of these represents a different category without any ordinal relationship. Therefore, we applied one hot encoding technique before applying our regression models. It means that each categorical feature with $m$ possible values will be transformed into $m$ binary features, with only one active at a time, as shown in table 4.2 . This step is necessary in order to apply our forecasting models as they only expect continuous input and would interpret the categories as being ordered, which is not desired. 
Feature scaling: We scale all the features from 0 to 1 using MinMax scaler function [89] of python. This is to make sure that our models assign appropriate weights to all the features, and do not overweight one of them just because they have high variance. For example, temperature in centigrade varies a little as compared to energy in watthour. So if we go without scaling, change in the co-efficient of energy by one unit change will have much stronger effect on the overall objective function as compared to one unit change in temperature.

Feature selection: Generally, this term is confused with feature extraction. However, feature extraction for us means to derive or extract more features from existing feature-set; and feature selection means to select a subset of features that are the best and are sufficient for the model to train. It is also useful because it helps to reduce the computational complexity and the running time of the algorithm.

From the original set of features described in Table 4.1, we already removed some correlated features in previous steps. In this step, we remove more features based on variance threshold approach, which is the baseline approach for feature selection. It removes all features whose variance is very low, for example in our case, pressure is almost constant. Its changes are very small and very random, so it does add anything beneficial for our model to learn.

Furthermore, we used recursive feature elimination (RFE) to select features by recursively considering smaller and smaller sets of features. In our case, we set the step size 0.25 which means that after every recursion, we remove $25 \%$ of the features. First, we train random forest estimator with 100 trees on the initial set of features and the importance of each feature is calculated. Then, we prune the $25 \%$ of the total features that are least important features. That procedure is recursively repeated on the pruned set until we are left with $25 \%$ of the total features. 
We selected random forest estimator because of its high performance to model complex relationships between the independent and dependent variables. We could have used simple Pearson correlation value to determine which feature has the strongest correlation, but it only gives us the strength of linear relationship between the feature and the target.

\subsubsection{Prediction Scenarios}

We forecast the energy consumption of different apartments for various lengths. Assuming a real-time data, we set the current date to December 1st, 2015 to forecast future data with NRMSE as general accuracy metric, unless stated otherwise. We use two different data prediction scenarios as follows:

\subsubsection{Scenario-1 (Daily data)}

Here we re-sample the data to daily interval and forecast for the following three scales. This work has been published in [5].

\section{- Small-scale prediction}

Use the past two week's data (2015-11-16 to 2015-11-30) to train our models and then use them to forecast the energy consumption for next three days (2015-12-01 to 2015-12-03).

\section{- Medium-scale prediction}

Use past one month's data (2015-11-01 to 2015-11-30) to train our models and then use them to forecast the energy consumption for next week (2015-12-01 to 2015-12-07).

\section{- Large-scale prediction}

Use past four month's data (2015-08-01 to 2015-11-30) to train our models and 
then use them to forecast the energy consumption for the next month (201512-01 to 2015-12-31).

\subsubsection{Scenario-2 (Half-hourly data)}

In scenario-1, we used daily data to forecast energy consumption. Most of the times utilities want to forecast energy consumption at higher resolution to design demand response algorithms. Therefore, in scenario-2, we will consider short-term forecasting using half-hourly data so that the utilities or consumers can forecast data for shortterms, for example, two hours ahead or six hours ahead, etc. As we discuss in the end, both scenarios have their own advantages. We cannot use half-hourly data to get an accurate forecast for months or years ahead, so we need daily data for that purpose. However, it is more appropriate to use half-hourly data or hourly data to solve day-ahead optimization algorithms.

As the daily data means relatively much less amount of data than half-hourly data, so we use simpler models like linear regression, support vector regression for daily data. We also compare their accuracy with FFNN model. As we increase the amount of data by using half hourly interval, we tend to use more complex models like LSTM neural network which are able to model complex relationships in a large amount of data.

\subsection{Results and Discussions}

\subsubsection{Scenario-1 (Daily data)}

The results for small-scale, medium-scale and large-scale predictions are shown in Fig. 4.10, Fig. 4.11 and Fig. 4.12, respectively. For simplicity, we only represent the graphs for energy consumption of Apartment 2. All these results are gathered using 
time and temperature as predicting features, with all six forecasting models.

Fig. 4.10 shows the small-scale prediction results. We observe that SVR using linear kernel gives the highest accuracy (NRMSE: 0.129). All other models are also fairly accurate with NRMSE ranging between 0.15 and 0.20 . On the other hand, SVR with Gaussian kernel is a high NRMSE of 0.36 .

In Fig. 4.11, we see that polynomial regression of order two gives a good accuracy with NRMSE of 0.147 when used for medium-scale prediction, i.e., forecasting energy consumption for the next week, using past two weeks of data. All other models show the error between 0.2 to 0.3 . The most accurate model for this scenario is found to be FFNN which gives NRMSE of 0.1383 .

For the large-scale prediction, i.e. forecasting energy consumption for the next month using past four months of data, we see that the FFNN model again surpasses all other models. It gives an error of 0.0754 as shown in Fig. 4.12, while all other models have errors ranging from 0.2 to 0.3 .

The accuracy of the presented models is summarized in Table 4.3 for different scales. In what follows, we highlight the performance of each prediction model for

Table 4.3: Evaluation of prediction models for Scenario-1 (Daily data).

\begin{tabular}{|c|c|c|c|c|c|c|c|c|c|}
\hline \multirow{2}{*}{ Prediction Models } & \multicolumn{3}{|c|}{ NRMSE } & \multicolumn{3}{|c|}{ NMAE } & \multicolumn{3}{|c|}{$R^{2}(\mathrm{COD})$} \\
\hline & $\mathrm{S}^{1}$ scale & $\mathrm{M}^{2}$ scale & $\mathbf{L}^{3}$ scale & $\mathrm{S}^{1}$ scale & $\mathrm{M}^{2}$ scale & $\mathbf{L}^{3}$ scale & $\mathrm{S}^{1}$ scale & $\mathrm{M}^{2}$ scale & $\mathbf{L}^{3}$ scale \\
\hline Linear Regression & 0.1776 & 0.2123 & 0.23382 & 0.156 & 0.2015 & 0.146 & 0.14 & 0.29 & 0.45 \\
\hline Polynomial Regression & 0.1588 & 0.1664 & 0.2273 & 0.1429 & 0.147 & 0.2754 & 0.14 & 0.3 & 0.57 \\
\hline SVR linear kernel & 0.115 & 0.1651 & 0.2160 & 0.1269 & 0.2736 & 0.159 & 0.13 & 0.24 & 0.44 \\
\hline SVR Polynomial & 0.1642 & 0.3519 & 0.244 & 0.1532 & 0.331 & 0.2252 & 0.12 & 0.19 & 0.54 \\
\hline SVR Gaussian model & 0.09491 & 0.22479 & 0.141 & 0.3613 & 0.202 & 0.163 & 0.37 & 0.46 & 0.64 \\
\hline FFNN & 0.1961 & 0.1383 & 0.0753 & 0.1633 & 0.0843 & 0.1154 & 0.18 & 0.59 & 0.49 \\
\hline
\end{tabular}




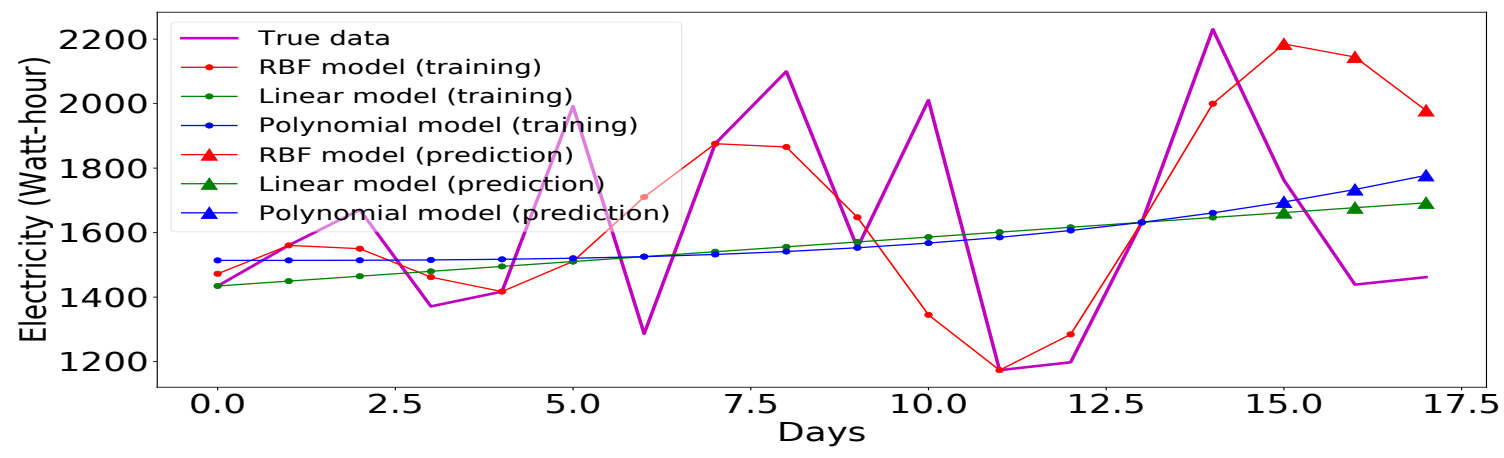

(a) Small-scale prediction using SVR.

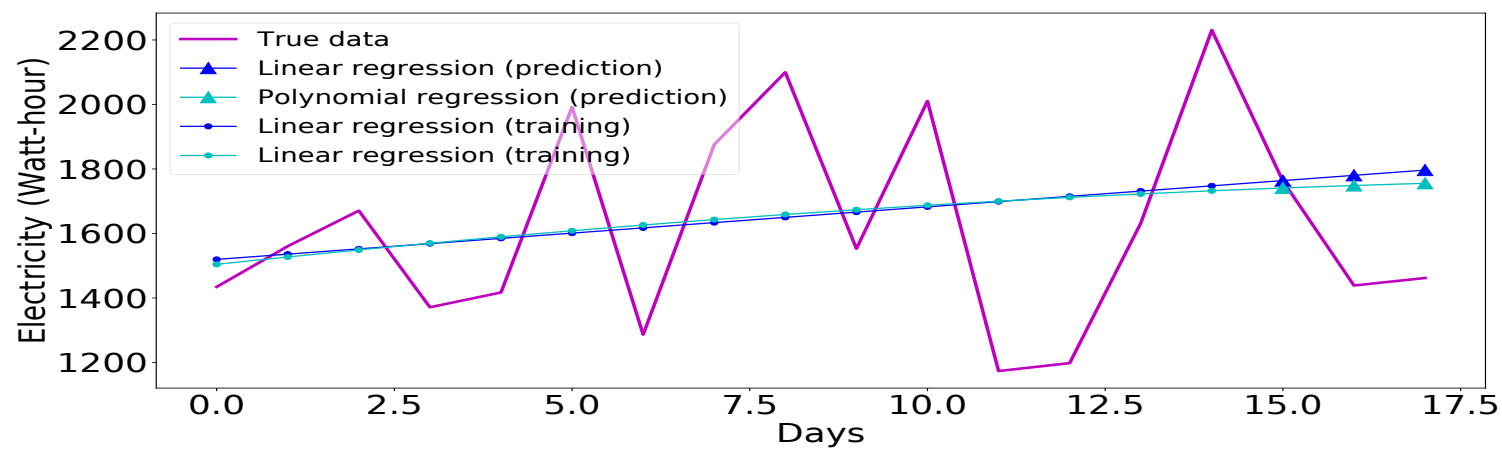

(b) Small-scale prediction using linear and polvnomial regression.

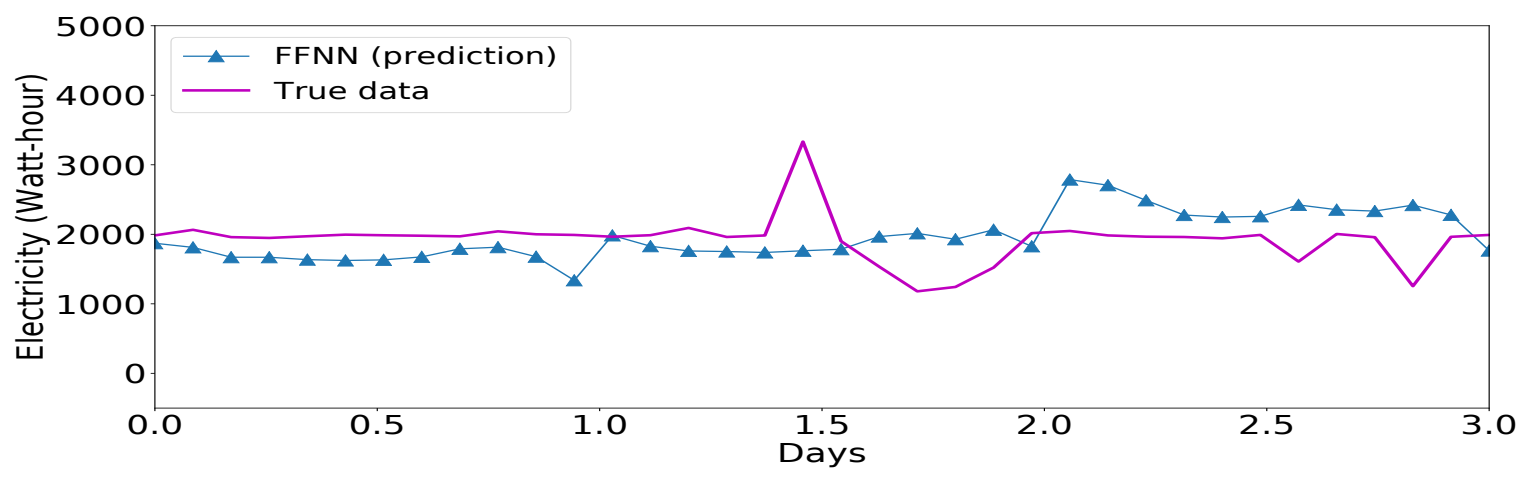

(c) Small-scale Prediction using FFNN.

Figure 4.10: Small-scale: predicting next three days based on past two weeks.

daily data.

Linear regression model gives the best results for small-scale prediction (NRMSE: 0.1777), i.e. when the energy consumption is forecasted based on short training period. As we increase the training period, this model gradually weakens and the 


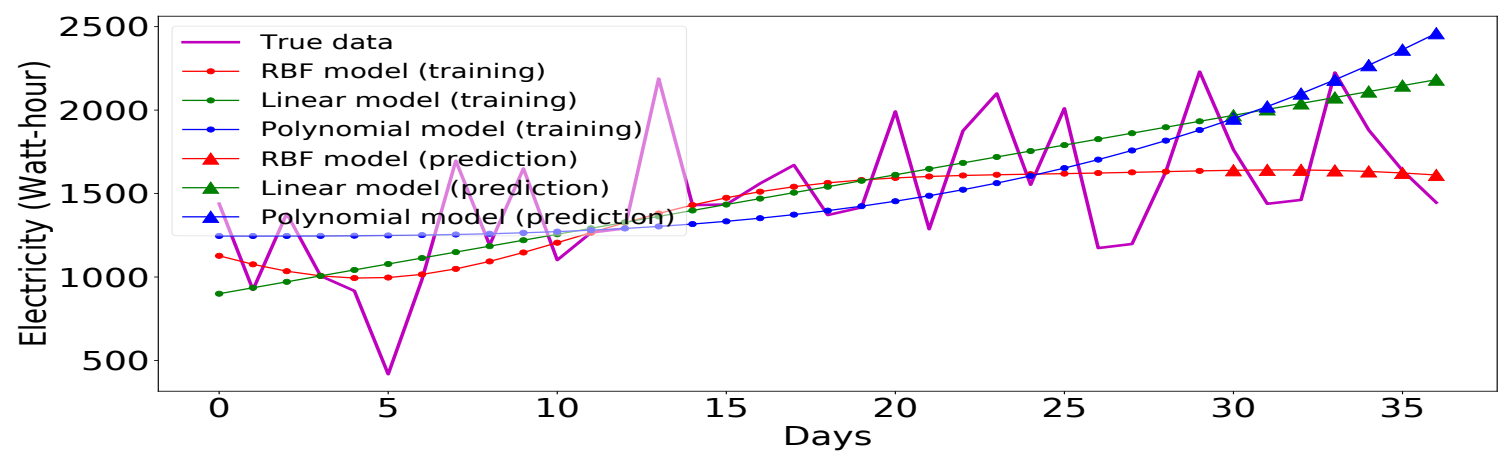

(a) Medium-scale prediction using SVR.

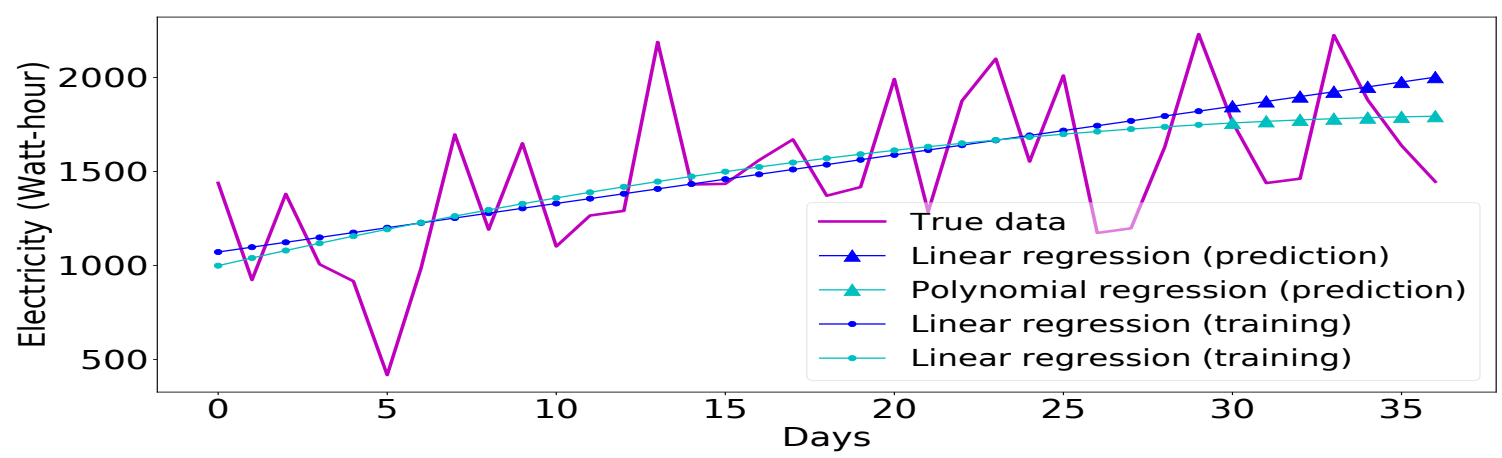

(b) Medium-scale prediction using linear and polvnomial reøression.

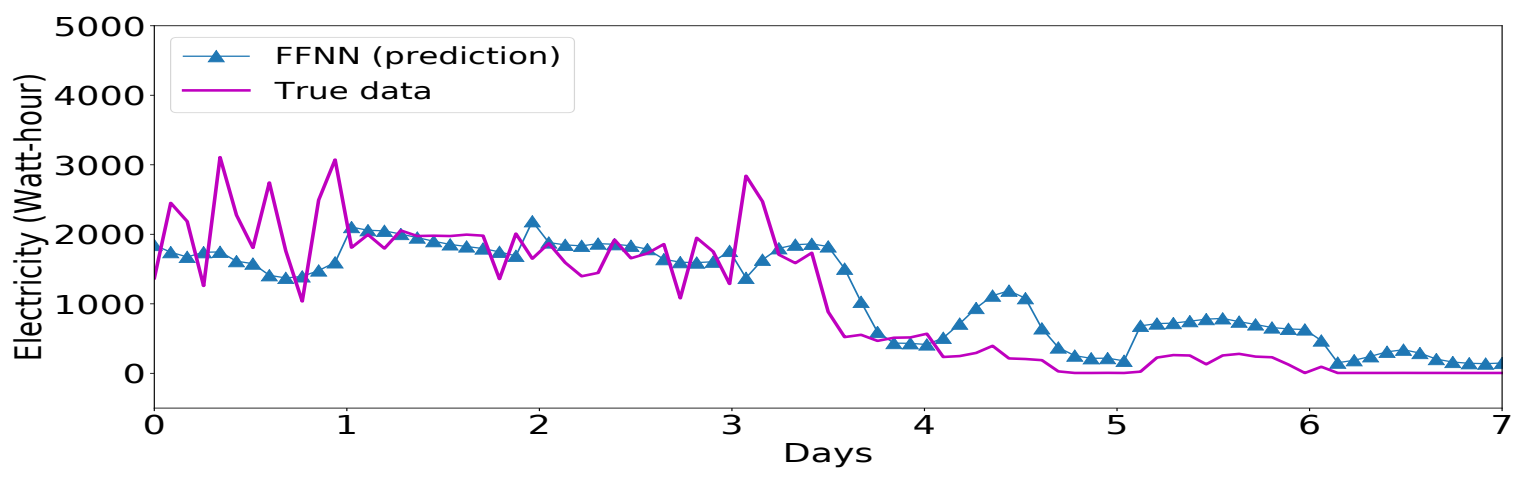

(c) Medium-scale Prediction using FFNN.

Figure 4.11: Medium-scale: predicting next week based on past month.

accuracy drops for large-scale prediction. The reason may be attributed to more fluctuations in large dataset which cannot be simply represented by a straight line.

For polynomial regression, the degree should increase with the data size to cater for more variations. For example, a polynomial of degree two fits well for medium-scale prediction, but we used degree three for large-scale. However, we can only increase 


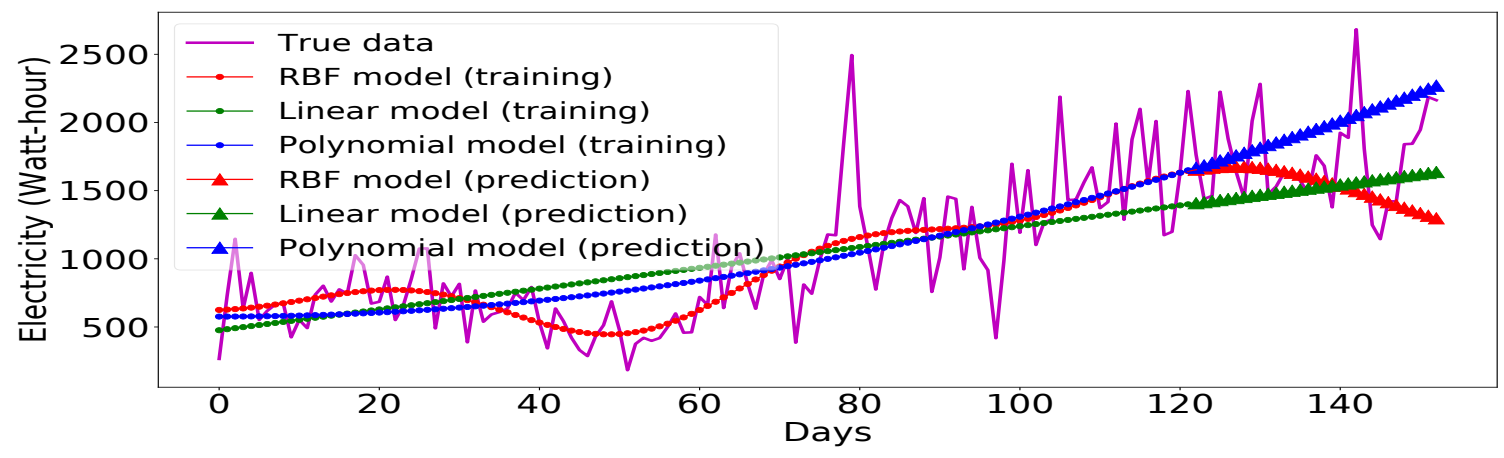

(a) Large-scale prediction using SVR.

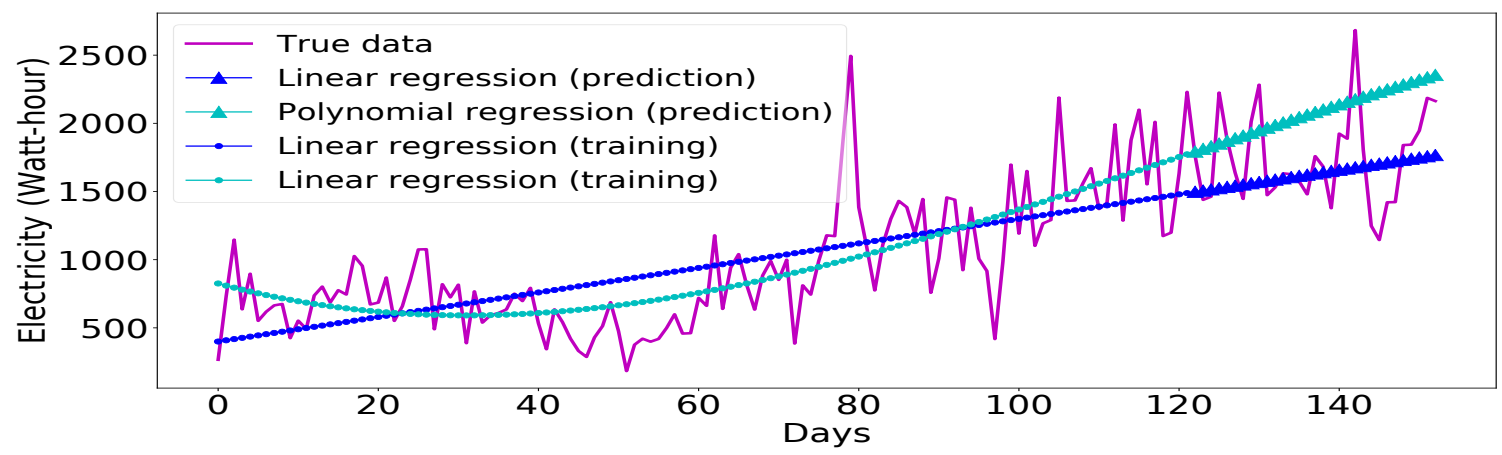

(b) Larce-scale prediction using linear and polvnomial regression.

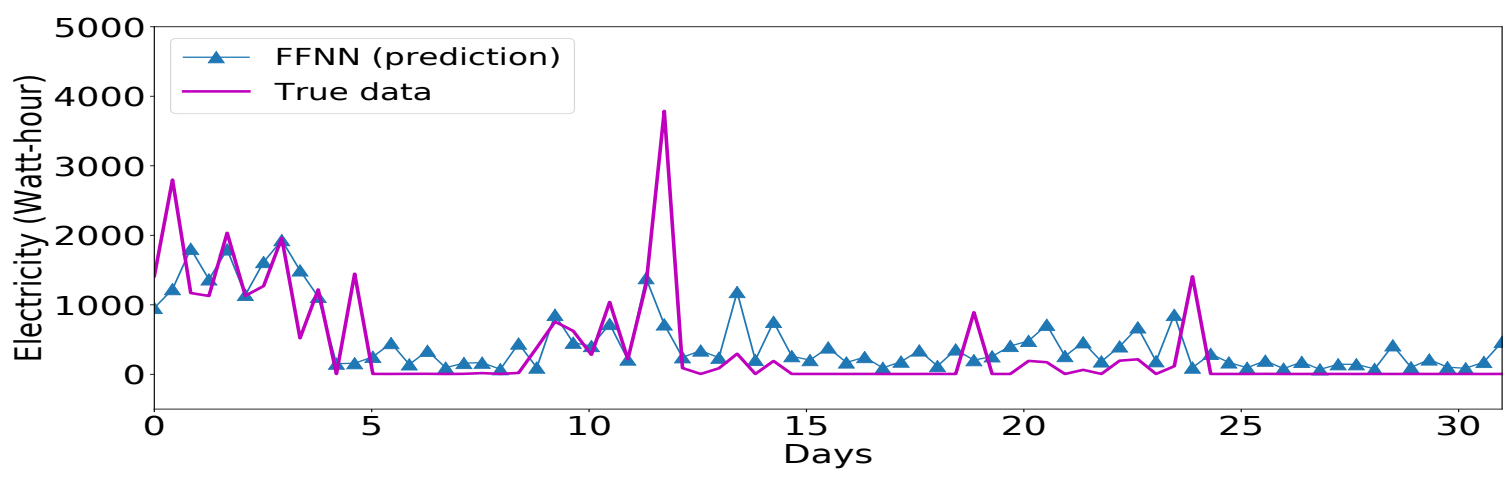

(c) Large-scale Prediction using FFNN.

Figure 4.12: Large-scale: predicting next month based on past four months

the degree up to a certain limit after which accuracy starts to drop, therefore we checked a range of values before selecting the optimum degree for each scenario.

SVR with linear kernel always achieves better accuracy than simple linear kernel. SVR with polynomial kernel with degree one is similar to the linear kernel. If we increase its degree to three, it becomes a better measure for forecasting than the linear 
kernel. If we go even higher, it becomes over-fit model which drops the accuracy and also makes the program too complex.

SVR with Gaussian kernel (SVR-G) is not a very accurate metric for small-scale prediction, however, its accuracy increases with the size of the dataset. The behaviour of this model is very sensitive to the tuning parameter $\gamma$. As the dataset increased in size, we used smaller $\gamma$ to achieve better accuracy. After checking for a wide range of possible values for gamma, we used 0.001 in large-scale, 0.01 in medium-scale, and 0.1 in small-scale for the best accuracy.

FFNN gives better accuracy in all scenarios except for small-scale prediction, where it has NRMSE of around 0.196. Its high accuracy can be attributed to its ability of mapping highly complex trends and patterns using the tanh activation function that quantifies any non-linearities in a model. As a result, we observe NRMSE of 0.139 for medium-scale prediction and 0.0754 for large-scale prediction scenarios.

\subsubsection{Scenario-2 (Half-hourly data)}

In scenario-2, we forecast short-term energy consumption using half hourly interval data. Initially, we forecast for next three days using history of three days and four months of data for training purpose at half-hourly interval. We then study the effect of changing different parameters of LSTM neural network and tune our model to get the best accuracy. Finally, we study the effect of changing history size (how many half-hours in past we are using as feature vectors) and forecasting size (how long we are forecasting). A part of this work has been published in [92]. In order to have real-time demand response, the resolution should be further increased, for example three seconds instead of half-hour, but such a high resolution is out of scope of this thesis.

Fig 4.13 shows the actual data from 1st Dec, 2015 till 3rd Dec, 2015 in cyan colour, 
and we compare it with the predictions obtained using LSTM model in magenta colour.

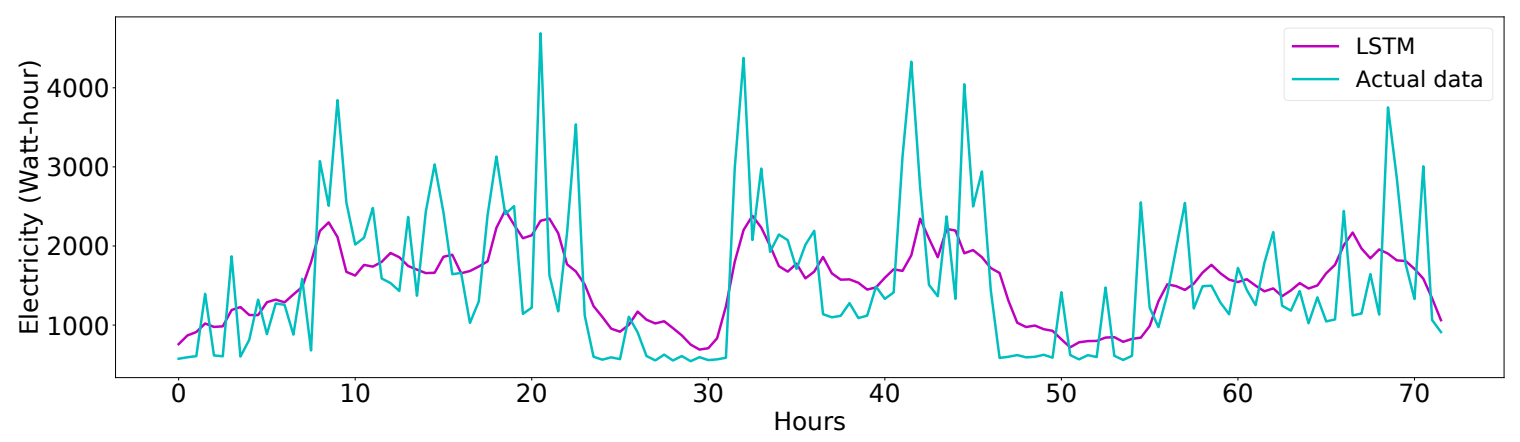

Figure 4.13: Short-term forecasting using LSTM.

\subsubsection{Tuning LSTM hyper-parameters}

Number of neurons and layers: We studied the effect of changing both these parameters and used grid-search method to find the optimal values in order to minimize the bias and the variance. Various combinations of neurons based on the input and output size are shown in Table 4.4. This list is nowhere exhaustive but gives an idea of various tested values. Our input size is the number of features and output size is the number of energy forecasting values which is always one, as we are predicting on rolling basis. In this particular case, we have input size of 720 . Please note that when we talk about the number of layers, we mean the number of hidden layers only, excluding the input and output layers.

Along with accuracy metrics, we also measured the execution time for each combination of neuron and layers. The results are shown in Fig 4.14. We notice that by adding more neurons, we tend to improve the accuracy in most cases, but up to a certain limit only, after which accuracy starts decreasing. In all three layer cases, the 


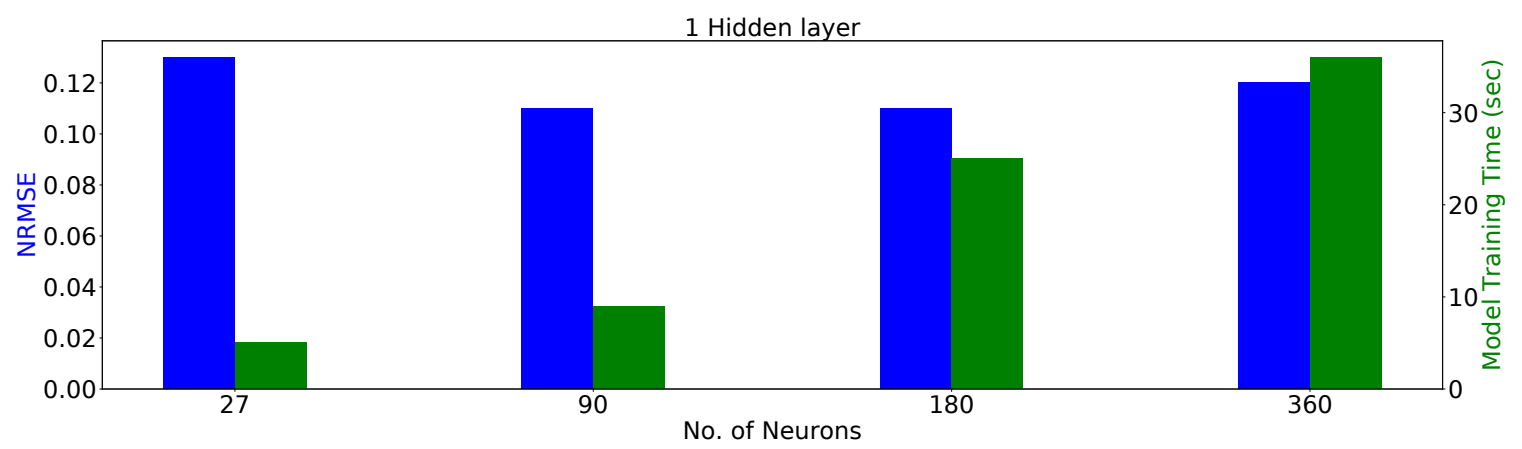

(a) Accuracy vs no. of neurons in one layer.

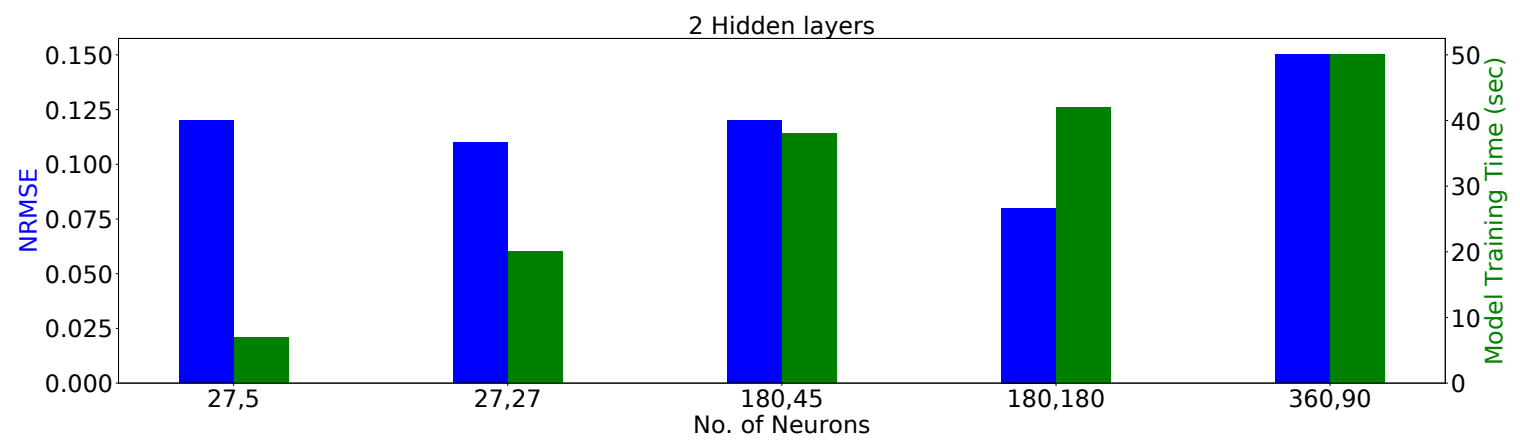

(b) Accuracy vs no. of neurons in two layers.

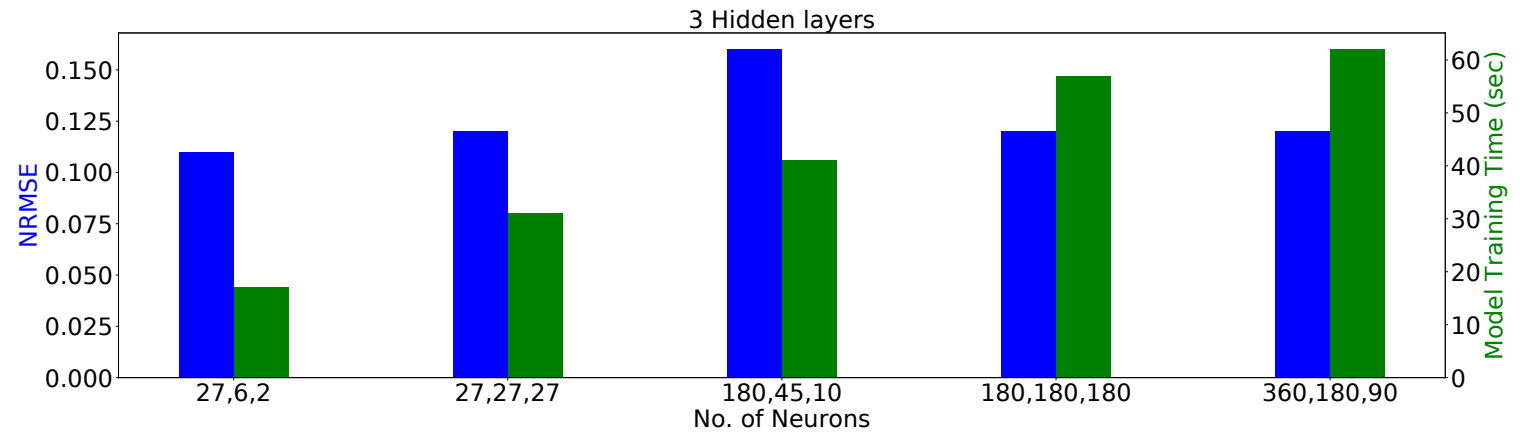

(c) Accuracy vs no. of neurons in three layers.

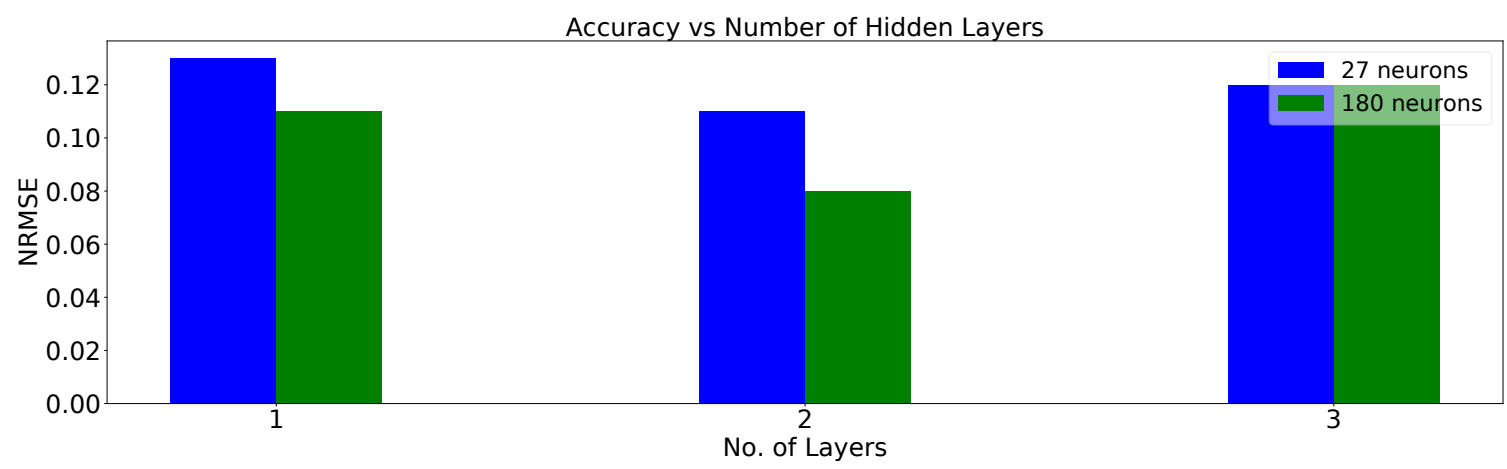

(d) Accuracy vs no. of layers for different number of neurons.

Figure 4.14: Effect of changing neurons and layers for LSTM. 
Table 4.4: Values considered for number of neurons and layers.

\begin{tabular}{|c|c|}
\hline Layers & No. of neurons \\
\hline One Layer & geometric mean (input size, output size) \\
arithmetic mean (input size, output size) \\
input size* $1 / 2$ \\
input size* $1 / 4$ \\
input size* $1 / 8$
\end{tabular}

best accuracy is obtained when we have the number of neurons equal to the quarter of the input size. We also notice from Fig 4.14 that accuracy improves by adding a second layer but then goes down when we add the third layer. By adding the fourth layer, our accuracy is the least and the model doesn't forecast anything, we just got a straight line for all the next half-hours. Based on this study, we conclude that we will use two layers, each having neurons equal to quarter of input size, as this gives us the best accuracy.

Optimization algorithm to minimize Loss: Optimization algorithms help us to minimize the loss function which in our case is mean square error. In neural network, 
weights and the bias values are its internal parameters which are used in computing the output values, and are learned and updated in the direction of optimal solution i.e minimizing the Loss by the network's training process. The gradient error is basically calculated using derivative of error with respect to the network weights.

Stochastic Gradient Descent (SGD) optimizer is the most popular technique used to do weights updates in a neural network model and solve its convex optimization problem. But it comes with challenges such as vanishing Learning rate, slow convergence or High variance in the parameter updates which leads to fluctuating Loss function. To overcome these challenges of gradient descent and its variants, we use Adam (Adaptive Moment Estimation) optimizer [93]. Classical stochastic gradient descent maintains a single learning rate for all weight updates and it does not change during training. Adam method computes individual adaptive learning rates for different parameters from estimates of first and second moments of the gradients. Moreover, SGD updates the weights after passing each sample which makes it slow. On the other hand, Adam updates the weights after each batch. Therefore, we used Adam optimizer in our work. Comparison between accuracy obtained from Adam vs SGD optimizer while keeping other parameters constant (except epochs) is shown in Fig 4.15.

Learning rate: Remember that Adam and SGD are the methods we used to update our weights. But there is another important parameter which determines how fast the weights are updated. Choosing a proper learning rate can be difficult. A learning rate that is too small leads to painfully slow convergence i.e will result in small baby steps towards finding optimal parameter values which minimize loss. On the other hand, a too large learning rate can hinder convergence and cause the loss function to fluctuate around the minimum or even to diverge. To finalize the optimum value for 


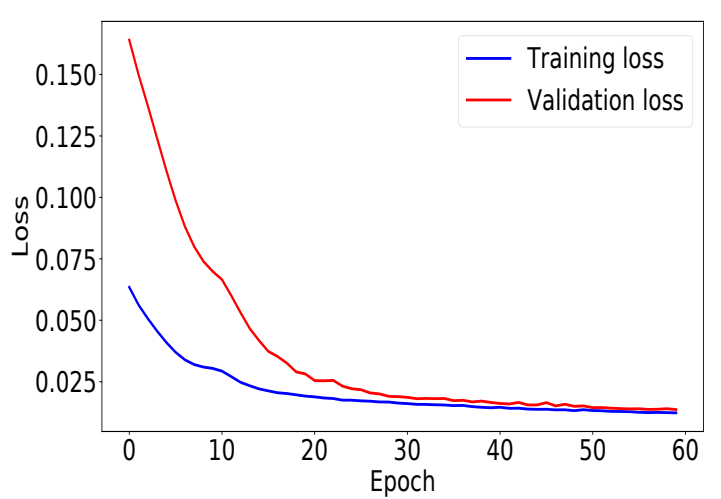

(a) Adam optimizer.

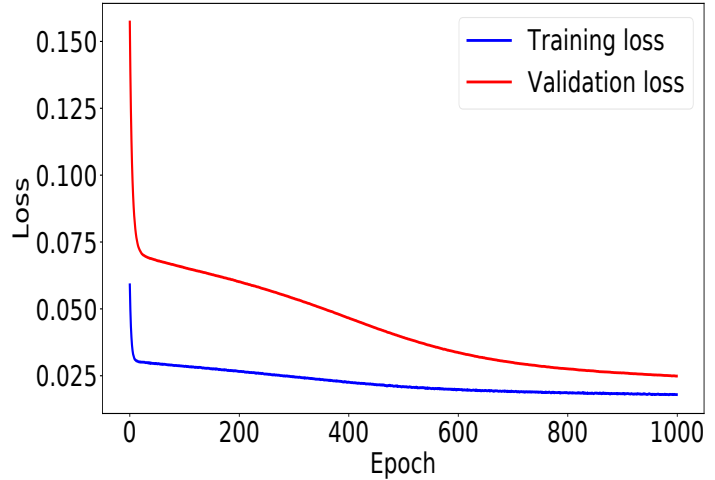

(b) SGD optimizer.

Figure 4.15: Comparison between Adam and SGD optimizer.

learning rate, we try its different values as shown in table 4.5. As the learning rate for Adam is adaptive, so when we specify the learning rate for Adam it is actually the learning rate at initial step, after which it adapts itself with weight updates.

We notice from the results that very slow learning rate takes a long time to process without accuracy improvement. High learning rate although converges quickly but gives poor accuracy metrics. The optimum value is 0.0001 where we get the least NRMSE. We also change the number of epochs for different learning rate to make sure the loss curve has converged. We see that the lower the learning rate, the more epochs it needs to converge the loss curve. We studied epochs between 15 and 1000 .

Table 4.5: Effect of learning rate.

\begin{tabular}{|c|c|c|}
\hline Learning Rate & NRMSE & No. of Epoch for which loss curve is converged \\
\hline $1 \mathrm{e}-1$ & 0.1763 & no convergence at any epoch because LR is too high \\
\hline $1 \mathrm{e}-2$ & 0.1233 & 15 \\
\hline $1 \mathrm{e}-3$ & 0.095 & 25 \\
\hline $1 \mathrm{e}-4$ & 0.096 & 60 \\
\hline $1 \mathrm{e}-5$ & 0.0984 & 200 \\
\hline $1 \mathrm{e}-6$ & 0.1354 & 1000 \\
\hline
\end{tabular}


Batch size and epoch: Batch size is the number of samples that our model will process in one forward/backward pass. The samples in a batch are processed independently, in parallel. For example, if we have 1000 training samples with a batch-size of 100, the algorithm takes the first 100 samples from the training dataset and trains the network. In parallel, it trains the network taking the next 100 samples. We keep doing this procedure until we have propagated through all samples of the network [94]. The higher the batch size, the more memory space is needed but faster is the training. If the batch size is equal to training data size, all the training data will be used once per epoch. If the batch size is one, every sample is processed one by one per epoch, which slows down the training but less memory of the machine will be used. In order to reach a compromise, we used the batch size equal to half of the number of lag variables used. This is to make sure that our model process half new and half old data in each forward/backward pass to update the weights after each batch.

Epoch is defined as one pass over the entire dataset. We train our model for multiple epochs in order to minimize the loss. Larger epoch means the model is trained for large number of times which decreases the loss, but after a certain limit of epoch, the reduction in the loss is negligible. In this work, we used epoch values from 5 to 1000 for different cases, for example, higher number of neurons required high epochs to converge the loss curve. Similarly, high learning rate only required five epochs to converge the loss curve. For every next pass, we shuffled our training batches, which means that the samples in each batch will be processed together, but the batch processed first in one epoch may be processed at last in the next epoch. This enables the model to learn various changing pattern in our time series data.

Dropout and regularization: LSTM models are a type of RNN that a capable of learning sequences of observations, but an issue with LSTMs is that they can easily 
overfit training data, reducing their predictive skill. Dropout [95] is a regularization method where input and recurrent connections to LSTM units are probabilistically excluded from activation and weight updates while training a network. This has the effect of reducing overfitting and improving model performance. Moreover, we do not use dropout for validation data, just for the training data. The reason is that during training we use drop out in order to add some noise for avoiding over-fitting. During cross-validation, we are in recall phase and use all the capabilities of the network.

We do not use the input dropout as we do not want to lose any inputs while training, however, in the subsequent layers, we use a dropout value of 0.2 which means for a given layer, $20 \%$ of the input features (randomly selected neurons) will be ignored/dropped out during training. This means that their contribution to the activation of downstream neurons is temporally removed on the forward pass and any weight updates are not applied to the neuron on the backward pass. This prevents any particular feature to overweigh and hence prevents overfitting. We also studied the effect of Lasso and Ridge Regularization but there was not any significant improvement in the accuracy. The range of dropout we used was from 0 to 0.7 , and we finally decided to only proceed with the dropout of 0.2 as it provided the best accuracy.

\subsubsection{History (window size of lags)}

After tuning our hyperparameters and deciding on the granularity, we study the effect of changing the history size. History is the number of lag variables we use as feature vectors in our model. This is very important as explained in detail in section 4.2.3. Based on the autocorrelations graphs shown in Fig 4.16, there is a very high correlation with two previous instances and the 24th lagged instance. In between them, the correlation decreases and then increases. The same pattern repeats if we 
increase the history where the correlation decreases after 24 lags and then increases and becomes high at 48 lags. This means that one-day history should be good enough for our model to predict the future. Adding further history means we are adding more correlated features which do not contribute positively to improve our accuracy. Figure 4.16 also shows that if we continue increasing the lag variables and plot autocorrelation, the value of maximum correlation decreases after each historical day. In

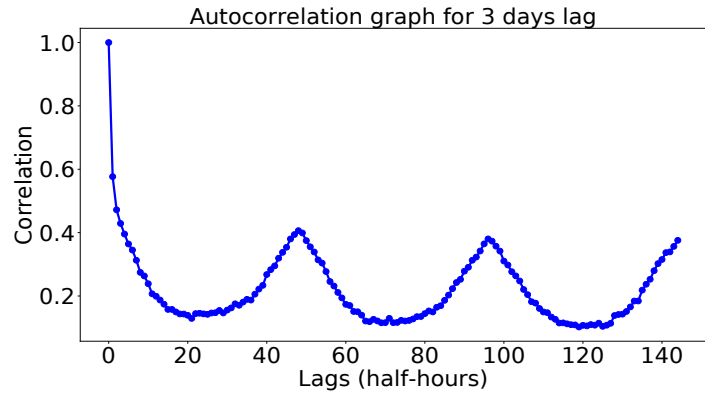

(a) Autocorrelation with 144 lags (3days).

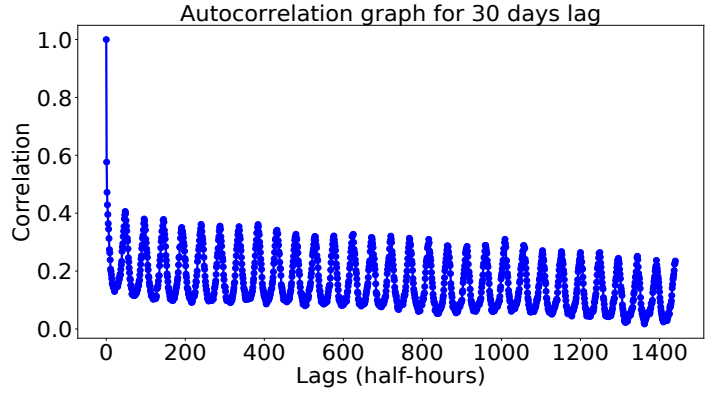

(b) Autocorrelation with 1440 lags (30days.)

Figure 4.16: Autocorrelation plot.

order to prove our hypothesis, we forecast for the following cases: (a) next 24 hours, (b) next 72 hours, (c) next 168 hours (7 days). In each of this case, we vary the history size from 1 hour to 168 hours with a step size of one hour.

As expected, we see that accuracy improves as we increase the history size for $24 \mathrm{hrs}$ forecast, but then the improvement becomes very slow or we do not see any improvement. The same trend is observed for $38 \mathrm{hrs}$ forecast. For the $72 \mathrm{hrs}$ forecast, we get some unexpected results. Accuracy improves up till next 24 hours as expected, but then continues to improve till 48 hours, after which we accuracy improves very slowly till 72 hours. The reason for this is that for $72 \mathrm{hrs}$ forecast, the model should have at least 72 hours of history as feature vectors so that while forecasting the $72^{\text {nd }}$ hour, it should know that was the value 72 hours ago. Although the addition of lagged variables after 24 hours reduce the accuracy, but based on the results we see that it 
is more important for the model to have at least the same amount of history as we want it to forecast. This is also proven when we forecast for seven days as shown in Fig 4.17. Based on our results, we conclude that we should use $X$ hours history to forecast next $X$ hours in order to get the best results.

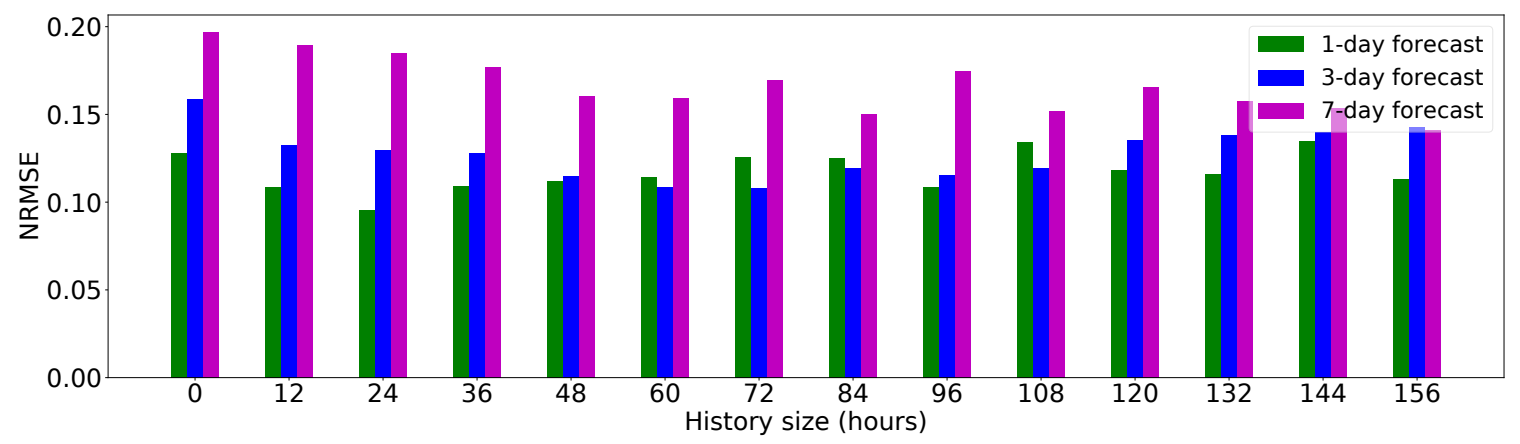

Figure 4.17: Accuracy vs history size for different forecasting sizes.

\subsubsection{Comparison with SVR}

From scenario-1, we observe that SVR-G has a very good performance after FFNN, and its accuracy improves as we increase the size of the dataset. Therefore, we compare our LSTM results with SVR-G as a baseline model in scenario-2. To tune our SVR-G model, we studied the effect of changing $\gamma$ and $c$ parameters and got the best results at $c=0.0001$, and $\gamma=10^{-5}$.

Fig 4.18 shows the actual data in cyan colour, and we compare it with the predictions obtained using LSTM and SVR-G models in magenta and blue colors respectively. Both models have been trained on past four months of data using history size of three days. We see that LSTM has slightly better overall performance than SVR-G model, specially for longer forecasts. 


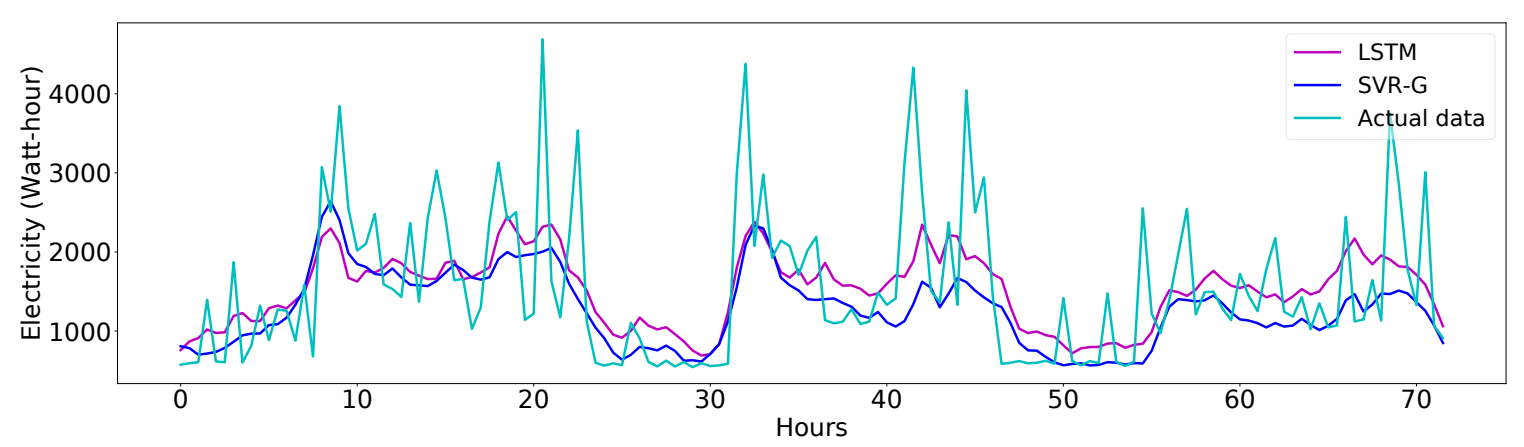

Figure 4.18: Short-term forecasting - LSTM vs SVR-G.

\subsubsection{Ensemble modelling}

As discussed in Section 2.3.2.5, ensemble modelling means combining the power of multiple models to achieve better results. After predicting the results through LSTM and SVR-G models, we propose our ensemble model for energy forecasting in which our base models are SVR-G and LSTM NN, and our meta-model is linear regression. Fig 4.19 shows the block diagram of our ensemble learning methodology. To the best

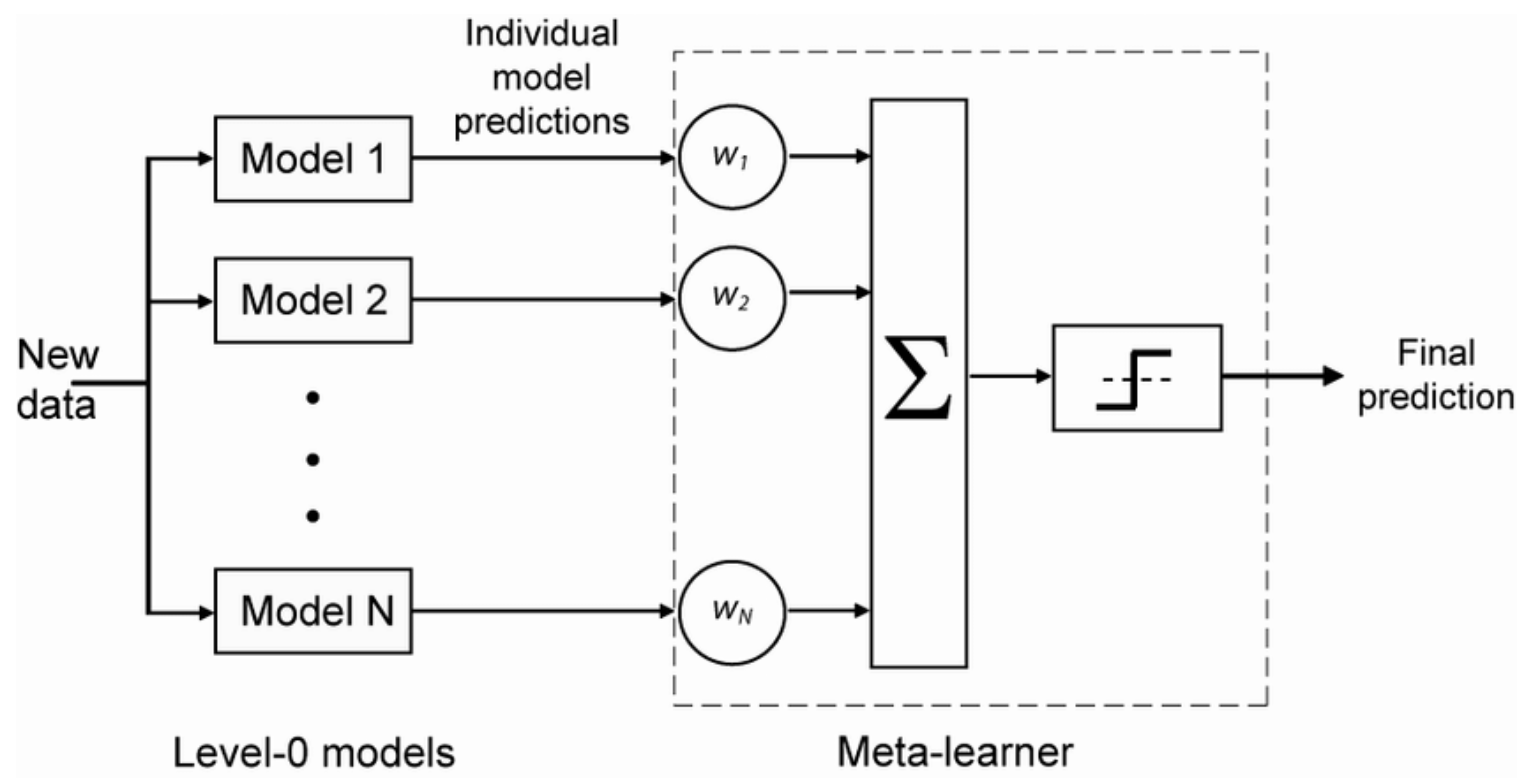

Figure 4.19: Stacking block diagram [96]. 
of our knowledge, this is the first time that an ensemble model based on LSTM and SVR-G is used for electricity forecasting with linear regression as s meta-model.

Comparison of results for the base models and ensemble model is shown in Fig 4.20 for 3-day forecast using 4-months of training data. It is evident that our proposed ensemble model is performing slightly better than the two base models. Table 4.6 provides details on how the accuracy changes for three comparative models for different lengths of forecasts. This is further elaborated in the following paragraph.

Table 4.6: Evaluation of prediction models for Scenario-2 (Half-hourly data).

\begin{tabular}{|c||c|c|c||c|c|c||c|c|c||}
\hline \hline \multicolumn{1}{||c||}{ Model } & \multicolumn{3}{c||}{ SVR-G } & \multicolumn{3}{c||}{ LSTM } & \multicolumn{3}{c||}{ Ensemble } \\
\hline Forecast Size & $\mathbf{R}^{2}$ & NMAE & NRMSE & $\mathbf{R}^{2}$ & NMAE & NRMSE & $\mathbf{R}^{2}$ & NMAE & NRMSE \\
\hline $2 \mathrm{~h}$ & -0.2369 & 0.0414 & 0.0435 & -0.0221 & 0.0299 & 0.039 & 0.4634 & 0.0252 & 0.0267 \\
\hline $6 \mathrm{~h}$ & 0.3235 & 0.0347 & 0.0411 & 0.2897 & 0.0308 & 0.0422 & 0.5793 & 0.0263 & 0.0312 \\
\hline $12 \mathrm{~h}$ & 0.6899 & 0.0672 & 0.0882 & 0.593 & 0.0722 & 0.1019 & 0.6881 & 0.0622 & 0.0885 \\
\hline $24 \mathrm{~h}$ & 0.4719 & 0.0907 & 0.1178 & 0.5203 & 0.0852 & 0.112 & 0.5754 & 0.0778 & 0.1051 \\
\hline $36 \mathrm{~h}$ & 0.6008 & 0.0832 & 0.112 & 0.6306 & 0.082 & 0.1075 & 0.7072 & 0.067 & 0.0951 \\
\hline $48 \mathrm{~h}$ & 0.4477 & 0.094 & 0.1345 & 0.5727 & 0.0904 & 0.1176 & 0.6359 & 0.0776 & 0.1081 \\
\hline $72 \mathrm{~h}$ & 0.408 & 0.0869 & 0.1269 & 0.5434 & 0.0858 & 0.1107 & 0.5927 & 0.0757 & 0.1042 \\
\hline \hline
\end{tabular}

Errors in rolling forecast: As we are using rolling forecast strategy to forecast energy consumption, we expect the errors to increase over each iteration. It is because the error in the first prediction accumulates with the error in the next prediction and so on. This is shown in Fig 4.21 in which we compare different types of error metrics for LSTM model, SVR-G model, and ensemble model. 


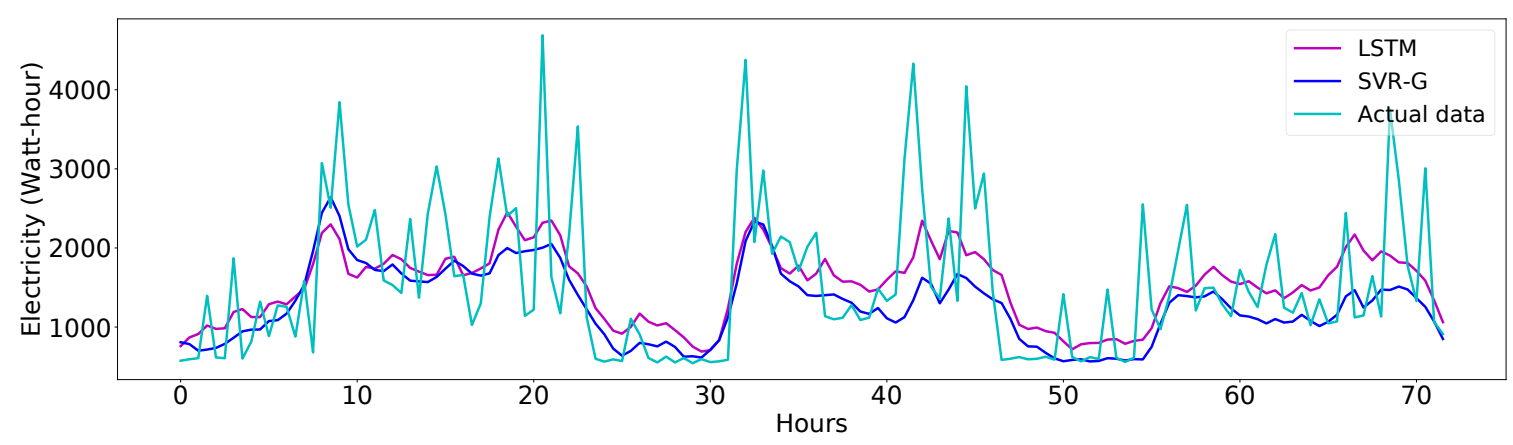

(a) Short-term forecasting - LSTM vs SVR-GLSTM vs SVR-G.

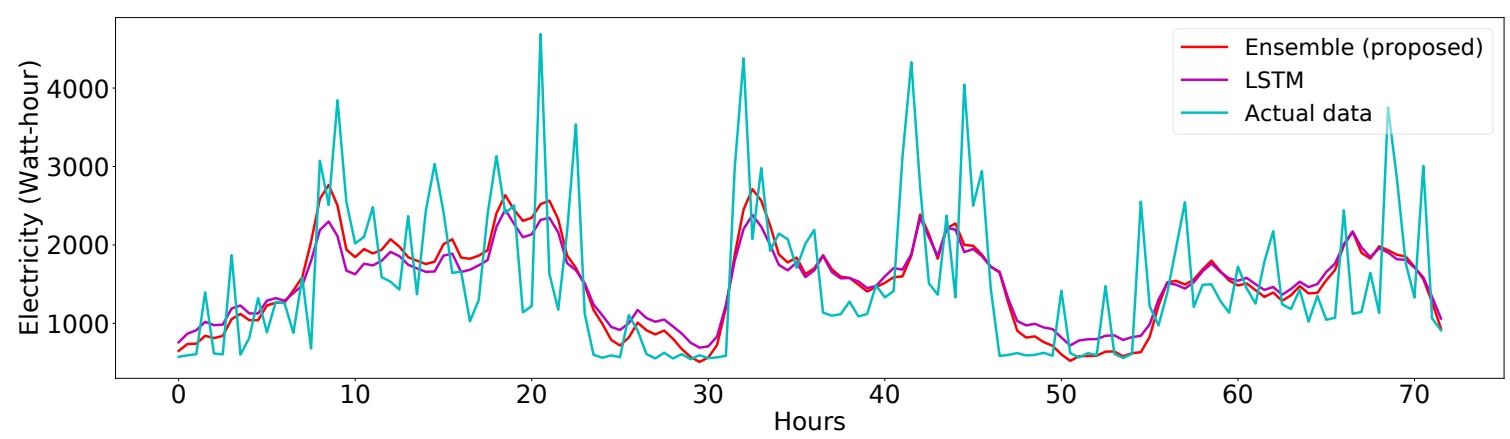

(b) Short-term forecasting - LSTM vs Ensemble.

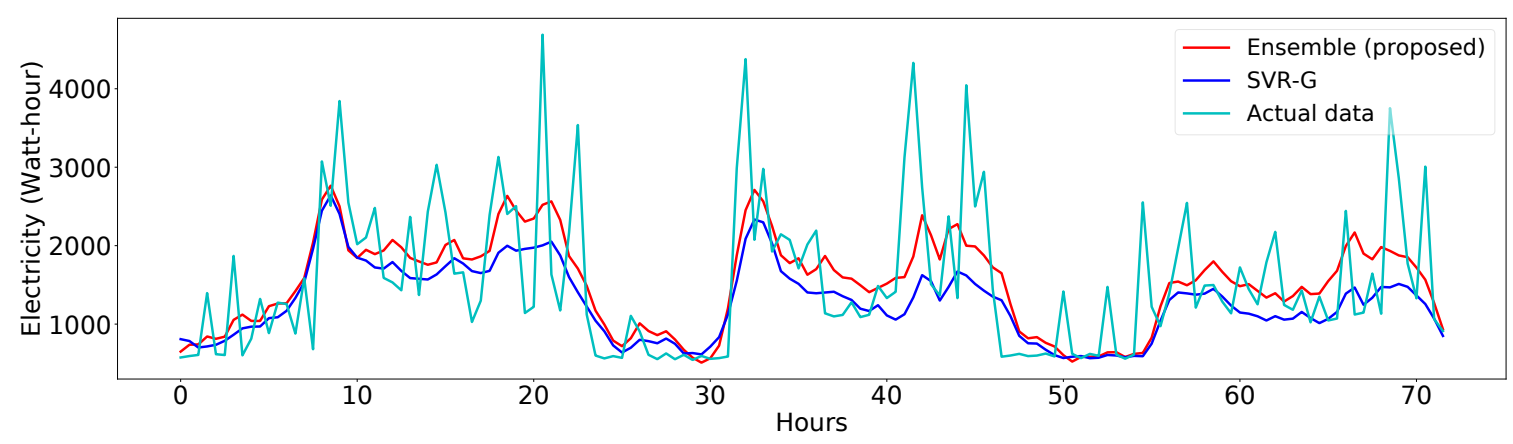

(c) Short-term forecasting - SVR-G vs Ensemble.

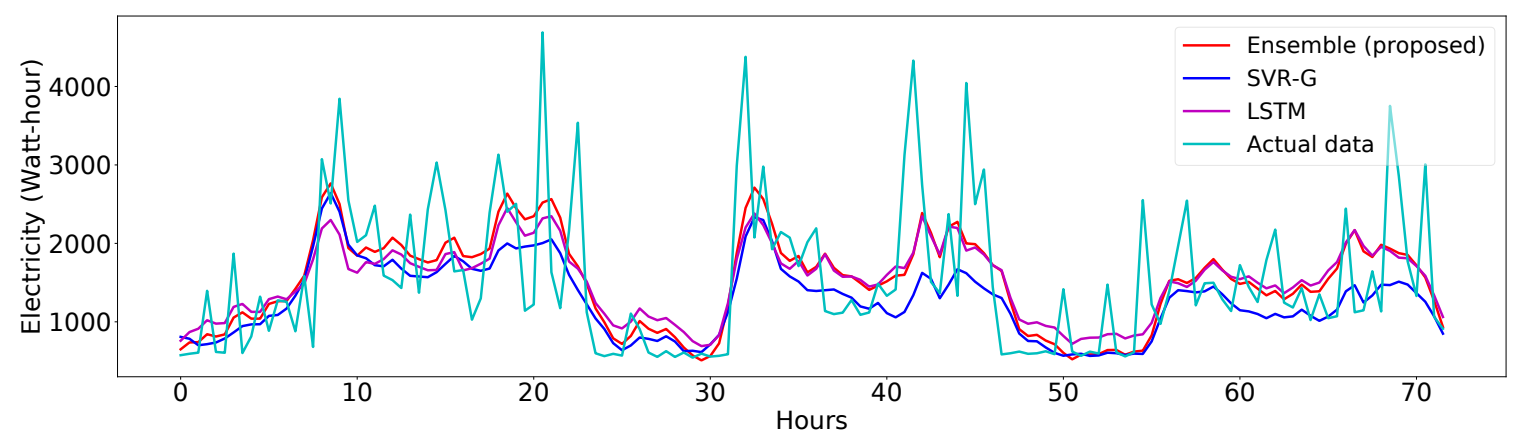

(d) Short-term forecasting - LSTM vs SVR-G vs Ensemble.

Figure 4.20: Performance of our proposed ensemble model vs existing models. 


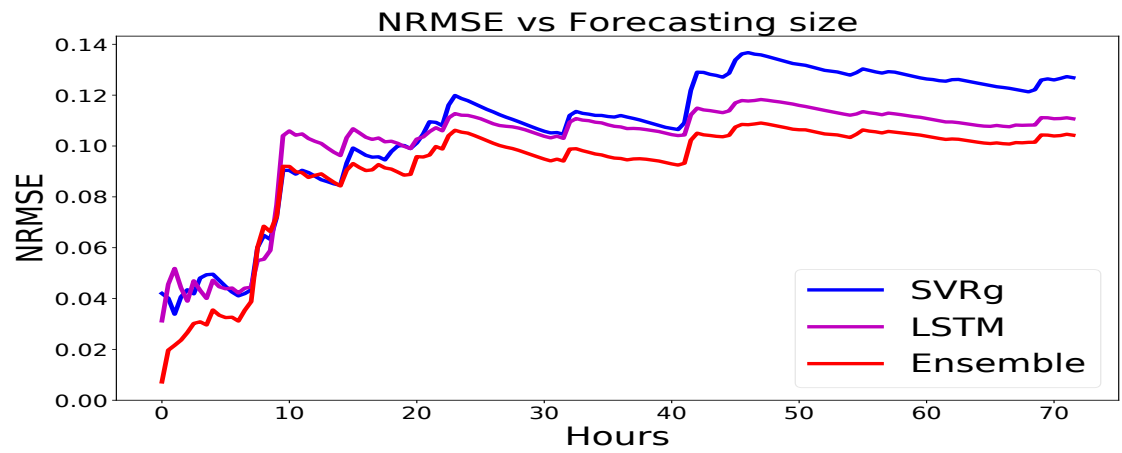

(a) Normalized root mean square error.

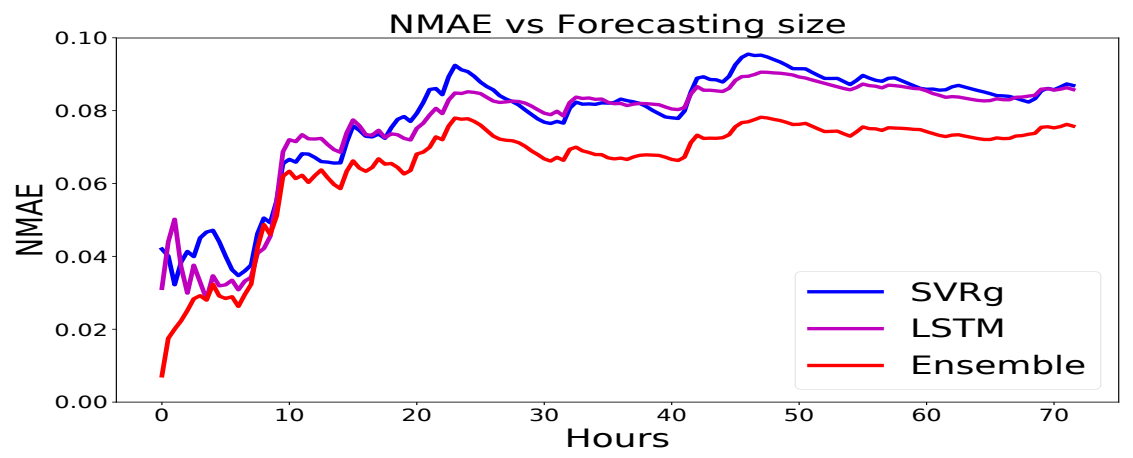

(b) Normalized mean absolute error.

Figure 4.21: Error vs forecasting size using different error metrics.

\subsubsection{Computation cost}

We observe from the Fig 4.21 and Table 4.6 that our proposed ensemble model outperforms the existing models, but there is a cost associated with it. This cost is the computational time, which increases multi folds when we combine various models because in this case we are training multiple models on two levels as opposed to a normal case where we only use one model at one level for prediction.

\subsection{Summary}

In this chapter, we performed energy forecasting using different models for short-term and long-term, and then presented our proposed approach based on ensemble models. 
We showed that our proposed approach has better performance than existing models, but its cost is the extra computational time.

In the next chapter, we provide the conclusion of this thesis along with proposed future directions. 


\section{Chapter 5}

\section{Conclusions and Future Work}

\subsection{Conclusions}

Smart grid technologies are transforming the utility sector all around the globe, however, it comes with many challenges. We focused on two important challenges, i.e., communications and data analytics in this thesis. Our main achievements and conclusions from this work are described below:

- A reliable mesh network was created that includes the electric transformer in the neighbourhood with a TA and different connected houses in the neighbourhood with CAs attached to them.

- Variety of field experiments were conducted where CAs were able to communicate their energy usage to the TA. The TA was also able to communicate action requests and information to all CAs. Communication was achieved via Wi-Fi in mesh mode. The network is IP-based, meaning that it is easily scalable and is compliant with IoT.

- The system is automated, resulting in minimal user interaction, and secure in terms of file encryption and secure remote access. Tests were done in real 
neighbourhood environments.

- Extreme conditions in terms of file sizes, physical obstacles and distances confirmed the system reliability. All the above results targeted the goal of being compliant with the IEEE 2030.5 standard's requirements.

- Communication availability ensured $98 \%$ of the time.

- Communication latency was well within the metric measurement goals, i.e., round trip: CA-TA $<1$-seconds; TA-BOS $<3$-seconds.

- In lab test at University of Ottawa, 10 negotiation cycles between TA and HEMSC were resolved in sub-second using our communication system.

- Lab testing and field trials have proven $100 \%$ communications and messaging exchange between TGDR elements (BOS, TA, CA, HEMSC).

- Based on results so far, TGDR can serve NIST and Federal Energy Regulatory Commission (FERC) requirements for Demand Response request to be sent and acknowledged within 30-seconds.

- In terms of data analytics, we tested various prediction models on a large dataset to forecast energy consumption of real houses.

- Proposed a novel approach for short-term energy forecasting by ensembling the LSTM and SVR-G models and applying a linear regression as a meta-model.

- Proved that our proposed approach performs better than the base models, but this comes at the cost of more computational time.

- Achieved normalized root mean square value of less than 0.05 for the short-term forecasting (up to 12 hours) and less than 0.09 for long-term forecasting (up to one month). 


\section{$5.2 \quad$ Future Work}

The insights obtained in this thesis are very promising, and we plan to build on these contributions in the following ways:

- Bringing the analytics to the Transformer Agent instead of the cloud, thus applying the concept of edge analytics. This will enable the TA to negotiate among themselves and between its CAs instead of sending all the data to the cloud.

- Upgrade the communication system from IPv4 to IPv6.

- Detect and predict anomalies in the consumer behaviours to make more informed decisions for the neighbourhood.

- Use static features in addition to the time series features, for example demographics of the residents, size or material of house. This further helps the utilities to design targeted programs for various clusters of consumers.

- Predict using high resolution data, for example, at three-second interval for real-time demand response. 


\section{List of Publications}

1. A. Ahmed, Z. Bouida and M. Ibnkahla, "Electricity Consumption Forecasting using Neural Networks", Data Day 5.0, Carleton University, June 2018. (Chapter 4)

2. A. Ahmed, K. Arab, Z. Bouida, and M. Ibnkahla, "Data Communication and Analytics for Smart Grid Systems," Proc 2018 IEEE International Conf. on Communications (ICC'18), Kansas City, MO, USA, pp. 1-6, May 2018.

(Chapter 3,4)

3. M. Ghalib, A. Ahmed, I. Al-Shiab, Z. Bouida, and M. Ibnkahla, "Implementation of a Smart Grid Communication System Compliant with IEEE 2030.5," 4th ICC Int. Workshop on Integrating Communications, Control, and Computing Technologies for Smart Grid, Kansas City, MO, USA, pp. 1-6, May 2018.

(Chapter 3)

4. Z. Bouida, J. Fattahi, A. Ahmed, M. Ibnkahla, H. Schriemer, and R. Abdullah, Smart Grid Communication Based on IEEE 2030 Standard, pp. 1-7, Springer International Publishing, 2018.

(Chapter 3, 4)

5. J. Kerkhoven, N. Charlebois, A. Robertson, B. Gibson, A. Ahmed, Z. Bouida, 
and M. Ibnkahla, "IPv6-Based Smart Grid Communication System over 6LoWPAN", submitted to IEEE WCNC'19, Apr 2019.

(Chapter 3)

6. A. Ahmed, Z. Bouida, and M. Ibnkahla, "Real Field Deployment and Testing of Smart Grid Communication System based on IEEE Smart Energy Profile 2.0", to be submitted to IEEE Transactions on Smart Grid, 2019.

(Chapter 3)

7. A. Ahmed, Z. Bouida, and M. Ibnkahla, "Electricity Forecasting using Ensemble model of LSTM and SVR", to be submitted to IEEE Transactions on Smart Grid, 2019.

(Chapter 4) 


\section{List of References}

[1] "Key findings and advance tables," Statistical Papers - United Nations (Ser. A), Population and Vital Statistics Report World Population Ageing 2015, p. 1-8, 2017.

[2] L. Gelazanskas and K. A. Gamage, "Demand side management in smart grid: A review and proposals for future direction," Sustainable Cities and Society, vol. 11, pp. 22-30, 2014.

[3] A. A. Munshi and A.-R. M. Yasser, "Big data framework for analytics in smart grids," Electric Power Systems Research, vol. 151, pp. 369-380, Oct 2017.

[4] Z. Bouida, J. Fattahi, A. Ahmed, M. Ibnkahla, H. Schriemer, and R. Abdullah, Smart Grid Communication Based on IEEE 2030 Standard, pp. 1-7. Cham: Springer International Publishing, 2018.

[5] A. Ahmed, K. Arab, Z. Bouida, and M. Ibnkahla, "Data communication and analytics for smart grid systems," in Proc. IEEE International Conference on Communications (ICC'2018), (Kansas City, MO, USA), pp. 1-6, May 2018.

[6] S. Mumtaz, A. Alsohaily, Z. Pang, A. Rayes, K. F. Tsang, and J. Rodriguez, "Massive internet of things for industrial applications: Addressing wireless iiot connectivity challenges and ecosystem fragmentation," IEEE Industrial Electronics Magazine, vol. 11, no. 1, pp. 28-33, 2017.

[7] J. Greenough, "'how the 'internet of things' will impact consumers, businesses, and governments in 2016 and beyond,"," Business Insider, 2016.

[8] D. Lund, C. MacGillivray, V. Turner, and M. Morales, "Worldwide and regional internet of things (iot) 2014-2020 forecast: A virtuous circle of proven value and demand," International Data Corporation (IDC), Tech. Rep, vol. 1, 2014. 
[9] N. Alliance, "5G white paper," Next generation mobile networks, white paper, pp. 1-125, 2015.

[10] M. Iwamura, "5G prospects-key capabilities to unlock digital opportunities," NGMN Alliance, Frankfurt, 2016.

[11] C. Mandil, "International energy agency," world energy outlook 2004," in International Energy Symposium, IEEJ, Tokyo, vol. 16, 2004.

[12] S. Aman, M. Frincu, C. Chelmis, M. Noor, Y. Simmhan, and V. K. Prasanna, "Prediction models for dynamic demand response: Requirements, challenges, and insights," in Smart Grid Communications (SmartGridComm), 2015 IEEE International Conference on, pp. 338-343, IEEE, 2015.

[13] S. Grid, "2030: A national vision for electricity's second 100 years," United States of America Department of Energy, 2003.

[14] IEEE, "IEEE adoption of smart energy profile 2.0 application protocol standard," IEEE Std 2030.5-2013, pp. 1-348, Nov 2013.

[15] "Save energy - residential - 'the great-dr' - hydro ottawa." https: //hydroottawa.com/save-energy/residential/great-dr. (Accessed on $11 / 06 / 2018)$.

[16] "Hydro ottawa." https://hydroottawa.com/. (Accessed on 11/06/2018).

[17] "Projects funded by the smart grid fund - ontario.ca." https://www . ontario . ca/document/projects-funded-smart-grid-fund. (Accessed on 11/06/2018).

[18] "Ldc tomorrow fund." https://secure2.eda-on.ca/imis15/EDA/Info_ Centre/LDC_Tomorrow_Fund.aspx. (Accessed on 11/06/2018).

[19] P. Heltzel, Complete wireless home networking. Prentice Hall Professional, 2003.

[20] "Wireless ad hoc network - wikipedia." https://en.wikipedia.org/wiki/ Wireless_ad_hoc_network. (Accessed on 11/06/2018).

[21] C. K. Toh, Ad hoc mobile wireless networks: protocols and systems. Pearson Education, 2001.

[22] M. Ibnkahla, Wireless Sensor Networks: A Cognitive Perspective. Boca Raton, FL, USA: Taylor and Francis Publishers, CRC Press, first ed., 2013. 
[23] A. Sabbah, A. El-Mougy, and M. Ibnkahla, "Routing protocols in smart grid," IEEE Trans. Ind. Informatics, Special Issue on Information Technologies in Smart Grids, vol. 10, pp. 210-221, Feb 2014.

[24] N. Saputro, K. Akkaya, and S. Uludag, "A survey of routing protocols for smart grid communications," Computer Networks, vol. 56, no. 11, pp. 2742 - 2771, 2012.

[25] T. H. Clausen and P. Jacquet, Optimized Link State Routing Protocol (OLSRP). RFC 3626, http://www.rfc-editor.org/info/rfc3626, Oct 2003.

[26] P. Jacquet, P. Muhlethaler, T. Clausen, A. Laouiti, A. Qayyum, and L. Viennot, "Optimized link state routing protocol for ad hoc networks," in Multi Topic Conference, 2001. IEEE INMIC 2001. Technology for the 21st Century. Proceedings. IEEE International, pp. 62-68, IEEE, 2001.

[27] T. Clausen and P. Jacquet, "Rfc 3626: Optimized link state routing protocol (olsr)," IETF, October, vol. 4, 2003.

[28] "Need to implement wireless device networking? here's what to look for ee times." https://www.eetimes. com/document.asp?doc_id=1279047. (Accessed on 11/14/2018).

[29] D.-M. Han and J.-H. Lim, "Design and implementation of smart home energy management systems based on zigbee," IEEE Transactions on Consumer Electronics, vol. 56, no. 3, 2010.

[30] Z. Zou, K.-J. Li, R. Li, and S. Wu, "Smart home system based on IPv6 and zigbee technology," Procedia Eng., vol. 15, pp. 1529-1533, 2011.

[31] A. Ghosh and N. Chakraborty, "Design of smart grid in an university campus using zigbee mesh networks," in IEEE Int. Conf. on Power Electronics, Intelligent Control and Energy Systems, pp. 1-6, 2016.

[32] N. Batista, R. Melício, J. Matias, and J. Catalão, "Photovoltaic and wind energy systems monitoring and building/home energy management using zigbee devices within a smart grid," Energy, vol. 49, pp. 306-315, 2013.

[33] M. H. Wen, K.-C. Leung, V. O. Li, X. He, and C.-C. J. Kuo, "A survey on smart grid communication system," APSIPA Transactions on Signal and Information Processing, vol. 4, 2015. 
[34] N. Saputro, K. Akkaya, and S. Uludag, "A survey of routing protocols for smart grid communications," Computer Networks, vol. 56, no. 11, pp. 2742-2771, 2012.

[35] F. Koohifar, N. Saputro, I. Guvenc, and K. Akkaya, "Hybrid wi-fi/lte aggregation architecture for smart meter communications," in Smart Grid Communications (SmartGridComm), 2015 IEEE International Conference on, pp. 575-580, IEEE, 2015.

[36] Y. Tsado, A. Gamage, K. Asanga, D. Lund, and B. Adebisi, "Performance analysis of variable smart grid traffic over ad hoc wireless mesh networks," 2016.

[37] A. A. Munshi and Y. A. I. Mohamed, "Cloud-based visual analytics for smart grids big data," in 2016 IEEE Power Energy Society Innovative Smart Grid Technologies Conference (ISGT), pp. 1-5, Sept 2016.

[38] J. Zheng, C. Xu, Z. Zhang, and X. Li, "Electric load forecasting in smart grids using long-short-term-memory based recurrent neural network," in Information Sciences and Systems (CISS), 2017 51st Annual Conference on, pp. 1-6, IEEE, 2017.

[39] S. B. Taieb, G. Bontempi, A. F. Atiya, and A. Sorjamaa, "A review and comparison of strategies for multi-step ahead time series forecasting based on the nn5 forecasting competition," Expert systems with applications, vol. 39, no. 8, pp. 7067-7083, 2012.

[40] R. Quiza and J. P. Davim, "Computational methods and optimization," in $M a-$ chining of hard materials, pp. 177-208, Springer, 2011.

[41] H. Liang, X. Sun, Y. Sun, and Y. Gao, "Text feature extraction based on deep learning: a review," EURASIP journal on wireless communications and networking, vol. 2017, no. 1, p. 211, 2017.

[42] "Basics of ensemble learning explained in simple english." https://www. analyticsvidhya.com/blog/2015/08/introduction-ensemble-learning/. (Accessed on 11/06/2018).

[43] S. Fortmann-Roe, "Understanding the bias-variance tradeoff," 2012.

[44] "Two is better than one: Ensembling models - towards data science." https://towardsdatascience.com/ two-is-better-than-one-ensembling-models-611ee4fa9bd8. (Accessed on 11/06/2018). 
[45] N. C. Dvornek, D. Yang, A. Venkataraman, P. Ventola, L. H. Staib, K. A. Pelphrey, and J. S. Duncan, "Prediction of autism treatment response from baseline fmri using random forests and tree bagging," arXiv preprint arXiv:1805.09799, 2018.

[46] M. Zareapoor and P. Shamsolmoali, "Boosting prediction performance on imbalanced dataset," International Journal of Information and Communication Technology, vol. 13, no. 2, pp. 186-195, 2018.

[47] R. Sikora et al., "A modified stacking ensemble machine learning algorithm using genetic algorithms," in Artificial Intelligence: Concepts, Methodologies, Tools, and Applications, pp. 395-405, IGI Global, 2017.

[48] M. Akour, I. Alsmadi, and I. Alazzam, "Software fault proneness prediction: a comparative study between bagging, boosting, and stacking ensemble and base learner methods," International Journal of Data Analysis Techniques and Strategies, vol. 9, no. 1, pp. 1-16, 2017.

[49] X. Qiu, Y. Ren, P. N. Suganthan, and G. A. Amaratunga, "Empirical mode decomposition based ensemble deep learning for load demand time series forecasting," Applied Soft Computing, vol. 54, pp. 246-255, 2017.

[50] K. Gajowniczek and T. Zabkowski, "Electricity forecasting on the individual household level enhanced based on activity patterns," PloS one, vol. 12, no. 4, p. e0174098, 2017.

[51] Y. Cheng, C. Xu, D. Mashima, V. L. Thing, and Y. Wu, "Powerlstm: Power demand forecasting using long short-term memory neural network," in International Conference on Advanced Data Mining and Applications, pp. 727-740, Springer, 2017.

[52] P. D. Diamantoulakis, V. M. Kapinas, and G. K. Karagiannidis, "Big data analytics for dynamic energy management in smart grids," Big Data Res., vol. 2, pp. 94-101, Sept 2015.

[53] Y. Simmhan, S. Aman, A. Kumbhare, R. Liu, S. Stevens, Q. Zhou, and V. Prasanna, "Cloud-based software platform for big data analytics in smart grids," Computing in Science \& Engineering, vol. 15, no. 4, pp. 38-47, 2013. 
[54] F. L. Quilumba, W.-J. Lee, H. Huang, D. Y. Wang, and R. L. Szabados, "Using smart meter data to improve the accuracy of intraday load forecasting considering customer behaviour similarities," IEEE Transactions on Smart Grid, vol. 6, pp. 911-918, Mar 2015.

[55] J. Kwac, J. Flora, and R. Rajagopal, "Household energy consumption segmentation using hourly data," IEEE Transactions on Smart Grid, vol. 5, no. 1, pp. 420-430, 2014.

[56] J. Kwac and R. Rajagopal, "Demand response targeting using big data analytics," in Big Data, 2013 IEEE International Conference on, pp. 683-690, IEEE, 2013.

[57] C.-Y. Kuo, M.-F. Lee, C.-L. Fu, Y.-H. Ho, and L.-J. Chen, "An in-depth study of forecasting household electricity demand using realistic datasets," in Proceedings of the 5th international conference on Future energy systems, pp. 145-155, ACM, 2014.

[58] X. Dong, L. Qian, and L. Huang, "Short-term load forecasting in smart grid: A combined CNN and K-means clustering approach," in Big Data and Smart Computing (BigComp), 2017 IEEE International Conference on, pp. 119-125, IEEE, 2017.

[59] W. Shen, V. Babushkin, Z. Aung, and W. L. Woon, "An ensemble model for dayahead electricity demand time series forecasting," in Proceedings of the fourth international conference on Future energy systems, pp. 51-62, ACM, Jan. 2013.

[60] H. Daki, A. El Hannani, A. Aqqal, A. Haidine, and A. Dahbi, "Big data management in smart grid: concepts, requirements and implementation," Journal of Big Data, vol. 4, no. 1, p. 13, 2017.

[61] R. E. Edwards, J. New, and L. E. Parker, "Predicting future hourly residential electrical consumption: A machine learning case study," Energy and Buildings, vol. 49, pp. 591-603, 2012.

[62] R. Shyam, B. G. HB, S. Kumar, P. Poornachandran, and K. Soman, "Apache spark a big data analytics platform for smart grid," Procedia Technology, vol. 21, pp. 171-178, 2015.

[63] J. Zheng, C. Xu, Z. Zhang, and X. Li, "Electric load forecasting in smart grids using long-short-term-memory based recurrent neural network," in Information 
Sciences and Systems (CISS), 2017 51st Annual Conference on, pp. 1-6, IEEE, 2017.

[64] C. Chelmis, J. Kolte, and V. K. Prasanna, "Big data analytics for demand response: Clustering over space and time," in Big Data (Big Data), 2015 IEEE International Conference on, pp. 2223-2232, IEEE, 2015.

[65] A. J. Conejo, J. M. Morales, and L. Baringo, "Real-time demand response model," IEEE Transactions on Smart Grid, vol. 1, no. 3, pp. 236-242, 2010.

[66] S. K. Sharma and X. Wang, "Live data analytics with collaborative edge and cloud processing in wireless iot networks," IEEE Access, vol. 5, pp. 4621-4635, 2017.

[67] I. F. Akyildiz and X. Wang, "A survey on wireless mesh networks," IEEE Radio Communications, vol. 43, p. 23-30, Sep 2005.

[68] B. Akyol, H. Kirkham, S. Clements, and M. Hadley, "A survey of wireless communications for the electric power system.," Prepared for the U.S. Department of Energy, Jan 2010.

[69] H. Gharavi and B. Hu, "Multigate communication network for smart grid," vol. 99, pp. 1028-1045, Jun 2011.

[70] J. Schneider, T. Kamiya, D. Peintner, and R. Kyusakov, "Efficient xml interchange (exi) format 1.0," W3C Proposed Recommendation, vol. 20, 2011.

[71] C. Glenn, D. Sterbentz, and A. Wright, "Cyber threat and vulnerability analysis of the u.s. electric sector,"

[72] Z. Kartit, A. Azougaghe, H. K. Idrissi, M. El Marraki, M. Hedabou, M. Belkasmi, and A. Kartit, "Applying encryption algorithm for data security in cloud storage," in Advances in Ubiquitous Networking, pp. 141-154, Springer, 2016.

[73] E. Rescorla, "Http over tls," tech. rep., 2000.

[74] T. Dierks and E. Rescorla, "Rfc 5246," The transport layer security (TLS) protocol version, vol. 1, 2008.

[75] E. Barker, "Suite b cryptography," 2006.

[76] C. NEMA, "250-enclosures for electrical equipment (1000 volts maximum)," National Electrical Manufacturers Association, 2003. 
[77] "Ad-hoc wireless mesh networking for the zombie apocalypse."

[78] "3g/4g \& lte base hat for raspberry pi - sixfab." https://sixfab.com/product/ raspberry-pi-3g-4glte-base-shield-v2/. (Accessed on 11/07/2018).

[79] "Quectel." https://www.quectel.com/product/ec25minipcie.htm. (Accessed on 11/07/2018).

[80] "Proxicast 4g/lte cross-polarized mimo 10 dbi high-gain fixed-mount panel antenna." https://www.amazon.com/ Proxicast-Cross-Polarized-High-Gain-Fixed-Mount-Antenna/dp/ B01NBSLNJ6. (Accessed on 11/27/2018).

[81] M. Ghalib, A. Ahmed, I. Al-Shiab, Z. Bouida, and M. Ibnkahla, "Implementation of a smart grid communication system compliant with IEEE 2030.5," in IEEE ICC 4th International Workshop on Integrating Communications, Control, and Computing Technologies for Smart Grid (ICT4SG), (Kansas City, MO, USA), pp. 1-6, May 2018.

[82] N. E. T. Nema, "Nov. 2005, 9 pages," National Electrical Manufacturers Association.

[83] C. A. Balanis and P. I. Ioannides, "Introduction to smart antennas," Synthesis Lectures on Antennas, vol. 2, no. 1, pp. 1-175, 2007.

[84] "Omni-directional antenna." http://bpastudio.csudh.edu/fac/lpress/471/ hout/wireless/omni.htm. (Accessed on 11/28/2018).

[85] "Low loss rf cable assemblies include sma, fme, tnc and others - siretta." https: //www.siretta.co.uk/low-loss-rf-cable-assemblies_f_92.php.

(Accessed on 11/28/2018).

[86] "Umasstracerepository. 2017. Smart data set for sustainability.."

[87] Y. Kim, J. Huang, and S. Emery, "Garbage in, garbage out: data collection, quality assessment and reporting standards for social media data use in health research, infodemiology and digital disease detection," Journal of medical Internet research, vol. 18, no. 2, 2016.

[88] G. E. Box, G. M. Jenkins, G. C. Reinsel, and G. M. Ljung, Time series analysis: forecasting and control. John Wiley \& Sons, 2015. 
[89] L. Buitinck, G. Louppe, M. Blondel, F. Pedregosa, A. Mueller, O. Grisel, V. Niculae, P. Prettenhofer, A. Gramfort, J. Grobler, R. Layton, J. VanderPlas, A. Joly, B. Holt, and G. Varoquaux, "API design for machine learning software: experiences from the scikit-learn project," in ECML PKDD Workshop: Languages for Data Mining and Machine Learning, pp. 108-122, 2013.

[90] C. Reviews, Business Statistics , For Contemporary Decision Making. Cram101, 2017.

[91] J. Benesty, J. Chen, Y. Huang, and I. Cohen, "Pearson correlation coefficient," in Noise reduction in speech processing, pp. 1-4, Springer, 2009.

[92] A. Ahmed, Z. Bouida, and M. Ibnkahla, "Electricity consumption forecasting using neural networks," Data Day 5.0, Carleton University, June 2018.

[93] D. P. Kingma and J. Ba, "Adam: A method for stochastic optimization," arXiv preprint arXiv:1412.6980, 2014.

[94] "python - what is batch size in neural network? - cross validated." https://stats.stackexchange.com/questions/153531/ what-is-batch-size-in-neural-network. (Accessed on 11/07/2018).

[95] N. Srivastava, G. Hinton, A. Krizhevsky, I. Sutskever, and R. Salakhutdinov, "Dropout: a simple way to prevent neural networks from overfitting," The Journal of Machine Learning Research, vol. 15, no. 1, pp. 1929-1958, 2014.

[96] M. T. Peiris, R. D. Jones, P. R. Davidson, and P. J. Bones, "Detecting behavioral microsleeps from eeg power spectra," in Engineering in Medicine and Biology Society, 2006. EMBS'06. 28th Annual International Conference of the IEEE, pp. 5723-5726, IEEE, 2006. 